\title{
Iron-Catalyzed Acylation of Polyfunctionalized Aryl- and Benzylzinc Halides with Acid Chlorides
}

\author{
Andreas D. Benischke, Marcel Leroux, Irina Knoll, and Paul Knochel* \\ Ludwig-Maximilians-Universität München, Department Chemie
}

Butenandtstraße 5-13, Haus F, 81377 München (Germany)

Fax: (+49) 089218077680

E-mail: Paul.Knochel@cup.uni-muenchen.de

\section{Table of contents:}

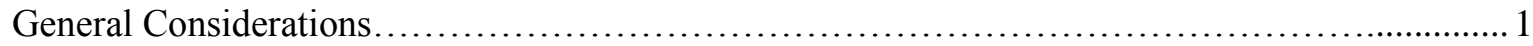

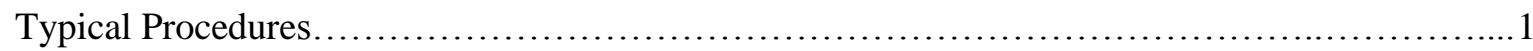

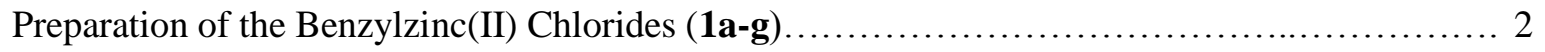

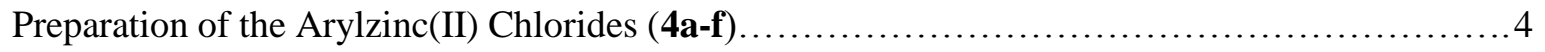

Iron-Catalyzed Acylation of Benzylic Zinc Chlorides (19-g) with Acid Chlorides.................. 6

Iron-Catalyzed Acylation of Arylzinc Chlorides (4a-f) with Acid Chlorides...................... 15

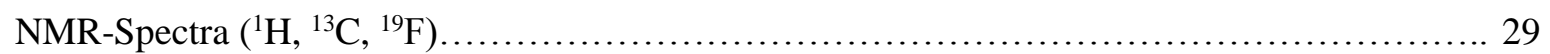

Additional Screenings of Additives, Catalysts and Polar Cosolvents............................. 63

Preliminary Experiments towards Iron-Catalyzed Acylation of Alkylzinc Halides $(\mathbf{6 a}, \mathbf{b}) \ldots \ldots \ldots 66$ 
General Considerations: All reactions were carried out under an argon atmosphere in flame-dried glassware. Syringes, which were used to transfer anhydrous solvents or reagents, were purged with argon prior to use. THF was continuously refluxed and freshly distilled from sodium benzophenone ketyl under nitrogen. Yields refer to isolated yields of compounds estimated to be $>95 \%$ pure as determined by ${ }^{1} \mathrm{H}$-NMR $\left(25{ }^{\circ} \mathrm{C}\right)$ and $\mathrm{GC}$-analysis. Chemical shifts are reported as $\delta$-values in ppm relative to the solvent peak. NMR spectra were recorded on solutions in $\mathrm{CDCl}_{3}$ (residual chloroform: $\delta=7.25 \mathrm{ppm}$ for ${ }^{1} \mathrm{H}-\mathrm{NMR}$ and $\delta=77.0 \mathrm{ppm}$ for ${ }^{13} \mathrm{C}-\mathrm{NMR}$ ). For the characterization of the observed signal multiplicities the following abbreviations were used: $s$ (singulet), $d$ (doublet), $t$ (triplet), dd (doublet of doublet), ddd (doublet of doublet of doublet), dt (doublet of triplet), dq (doublet of quartet), q (quartet), qn (quintet), m (multiplet) as well as br (broad). All reagents obtained from commercial sources were used without any further purification unless otherwise stated.

\section{Typical Procedure for the Preparation of Benzylzinc(II) Chlorides (1a-g) (TP 1):}

A dry and argon-flushed Schlenk-tube, equipped with a magnetic stirring bar and a rubber septum, was charged with $\mathrm{LiCl}\left(0.53 \mathrm{~g}, 12.5 \mathrm{mmol}, 1.25\right.$ equiv) and heated up to ca. $450{ }^{\circ} \mathrm{C}$ for $5 \mathrm{~min}$ under high vacuum using a heat gun. After cooling to room temperature under vigorous stirring, $\mathrm{ZnCl}_{2}(1.51 \mathrm{~g}$, $11.0 \mathrm{mmol}, 1.10$ equiv) was added under argon, the Schlenk-tube was heated to ca. $320^{\circ} \mathrm{C}$ for $5 \mathrm{~min}$ using a heatgun, cooled to room temperature and charged with magnesium turnings $(0.58 \mathrm{~g}, 24.0 \mathrm{mmol}$, 2.40 equiv). Freshly distilled THF (10-15 mL) and the corresponding benzylic chloride (10.0 mmol, 1.00 equiv) were added and the reaction mixture was stirred at room temperature for a given time until full conversion of the starting material was observed. The completion of the metalation was monitored by GC-analysis of hydrolyzed and iodolyzed aliquots. When the oxidative insertion was complete, the solution of the corresponding benzylzinc(II) chloride was separated from the resulting salts via a syringe equipped with a filter and transferred to another pre-dried and argon-flushed Schlenk-tube, before being titrated against iodine.

\section{Typical Procedure for the Preparation of Arylzinc(II) Chlorides (4a-f) (TP 2):}

A dry and argon-flushed Schlenk-tube, equipped with a magnetic stirring bar and a rubber septum, was charged with the corresponding aryl iodide $(10.0 \mathrm{mmol}, 1.00$ equiv) and dissolved in freshly distilled THF $(10 \mathrm{~mL})$. The solution was cooled to $-20^{\circ} \mathrm{C}$ and $i \operatorname{PrMgCl} \cdot \mathrm{LiCl}(10.0 \mathrm{~mL}, 12.0 \mathrm{mmol}, 1.21 \mathrm{M}$ in THF, 1.20 equiv) was dropwise added. The reaction mixture was stirred for a given time at the prior adjusted temperature until full conversion of the starting material was observed. The completion of the metalation was monitored by GC-analysis of hydrolyzed and iodolyzed aliquots with regard to an internal standard, transmetalated with $\mathrm{ZnCl}_{2}(12.0 \mathrm{~mL}, 12.0 \mathrm{mmol}, 1.00 \mathrm{M}$ in THF, 1.20 equiv) and finally titrated against iodine. 


\section{Typical Procedure for the Iron-Catalyzed Acylation of Benzylic Zinc Chlorides (1a-g) with Acid Chlorides (TP 3):}

A dry and argon-flushed Schlenk-tube, equipped with a magnetic stirring bar and a rubber septum, was charged with $\mathrm{FeCl}_{2}(6.34 \mathrm{mg}, 0.05 \mathrm{mmol}, 5.0 \mathrm{~mol} \%)$, the selected acid chloride (1.00 mmol, 1.00 equiv) and freshly distilled THF (1 mL). The corresponding prior prepared benzylic zinc chloride solution (1.25 mmol, 1.25 equiv) was dropwise added and the reaction mixture was stirred for a given time at room temperature. Subsequently, the reaction mixture was quenched with a saturated aqueous $\mathrm{NH}_{4} \mathrm{Cl}$ solution and the aqueous layer was extracted with EtOAc $(3 \times 75 \mathrm{~mL})$. The combined organic layers were dried over $\mathrm{MgSO}_{4}$, filtered and concentrated under reduced pressure. Purification of the crude products by flash column chromatography afforded the desired products.

\section{Typical Procedure for the Iron-Catalyzed Acylation of Arylzinc Chlorides (4a-f) with Acid Chlorides (TP 4):}

A dry and argon-flushed Schlenk-tube, equipped with a magnetic stirring bar and a rubber septum, was charged with $\mathrm{FeCl}_{2}(6.34 \mathrm{mg}, 0.05 \mathrm{mmol}, 5.0 \mathrm{~mol} \%)$, the selected acid chloride (1.00 mmol, 1.00 equiv) and freshly distilled THF $(1 \mathrm{~mL})$. The corresponding prior prepared arylzinc chloride $(1.25 \mathrm{mmol}$, 1.25 equiv) was dropwise added and the reaction mixture was stirred for a given time at $50{ }^{\circ} \mathrm{C}$. Subsequently, the reaction mixture was quenched with a saturated aqueous $\mathrm{NH}_{4} \mathrm{Cl}$ solution and the aqueous layer was extracted with EtOAc $(3 \times 75 \mathrm{~mL})$. The combined organic layers were dried over $\mathrm{MgSO}_{4}$, filtered and concentrated under reduced pressure. Purification of the crude products by flash column chromatography afforded the desired products.

\section{Preparation of the Benzylzinc(II) chlorides (1a-g)}

\section{Preparation of benzylzinc(II) chloride (1a)}

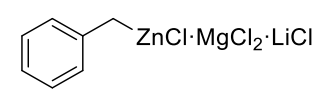

According to TP1, $\mathrm{LiCl}\left(0.53 \mathrm{~g}, 12.5 \mathrm{mmol}, 1.25\right.$ equiv), $\mathrm{ZnCl}_{2}(1.51 \mathrm{~g}, 11.0 \mathrm{mmol}, 1.10$ equiv), $\mathrm{Mg}$ turnings ( $0.58 \mathrm{~g}, 24.0 \mathrm{mmol}, 2.40$ equiv) and freshly distilled THF (10 mL) were used. Benzyl chloride $(1.27 \mathrm{~g}, 1.16 \mathrm{~mL}, 10.0 \mathrm{mmol}, 1.00$ equiv) was dropwise added and the reaction mixture was stirred for $1 \mathrm{~h}$ at room temperature. The concentration of benzylzinc(II) chloride (1a) was determined by titration with iodine in THF $(0.80 \mathrm{M}, 80 \%)$. 


\section{Preparation of 3-(trifluoromethyl)benzylzinc(II) chloride (1b)}

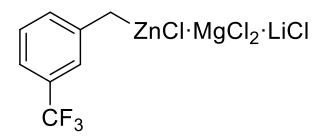

According to TP1, $\mathrm{LiCl}\left(0.53 \mathrm{~g}, 12.5 \mathrm{mmol}, 1.25\right.$ equiv), $\mathrm{ZnCl}_{2}(1.51 \mathrm{~g}, 11.0 \mathrm{mmol}, 1.10$ equiv), $\mathrm{Mg}$ turnings (0.58 g, $24.0 \mathrm{mmol}, 2.40$ equiv) and freshly distilled THF $(10 \mathrm{~mL})$ were used. 3-(Trifluoromethyl)benzyl chloride (1.95 g, $1.56 \mathrm{~mL}, 10.0 \mathrm{mmol}, 1.00$ equiv) was dropwise added and the reaction mixture was stirred for $2 \mathrm{~h}$ at room temperature. The concentration of 3-(trifluoromethyl)benzylzinc(II) chloride (1b) was determined by titration with iodine in THF $(0.72 \mathrm{M}, 72 \%)$.

\section{Preparation of 3-fluorobenzylzinc(II) chloride (1c)}

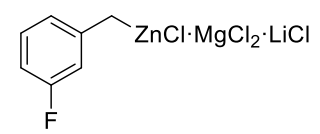

According to TP1, $\mathrm{LiCl}$ (0.53 g, $12.5 \mathrm{mmol}, 1.25$ equiv), $\mathrm{ZnCl}_{2}(1.51 \mathrm{~g}, 11.0 \mathrm{mmol}, 1.10$ equiv), $\mathrm{Mg}$ turnings ( $0.58 \mathrm{~g}, 24.0 \mathrm{mmol}, 2.40$ equiv) and freshly distilled THF (10 mL) were used. 3-Fluorobenzyl chloride (1.45 g, $1.21 \mathrm{~mL}, 10.0 \mathrm{mmol}, 1.00$ equiv) was dropwise added and the reaction mixture was stirred for $2 \mathrm{~h}$ at room temperature. The concentration of 3-fluorobenzylzinc(II) chloride (1c) was determined by titration with iodine in THF $(0.65 \mathrm{M}, 65 \%)$.

\section{Preparation of 3-(ethoxycarbonyl)benzylzinc(II) chloride (1d)}

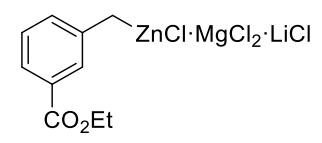

According to TP1, $\mathrm{LiCl}\left(0.53 \mathrm{~g}, 12.5 \mathrm{mmol}, 1.25\right.$ equiv), $\mathrm{ZnCl}_{2}(1.51 \mathrm{~g}, 11.0 \mathrm{mmol}, 1.10$ equiv), $\mathrm{Mg}$ turnings $(0.58 \mathrm{~g}, 24.0 \mathrm{mmol}, 2.40$ equiv) and freshly distilled THF (15 mL) were used. Ethyl 3(chloromethyl)benzoate ( $1.99 \mathrm{~g}, 1.70 \mathrm{~mL}, 10.0 \mathrm{mmol}, 1.00$ equiv) was dropwise added and the reaction mixture was stirred for $1.5 \mathrm{~h}$ at room temperature. The concentration of 3-(ethoxycarbonyl)benzylzinc(II) chloride (1d) was determined by titration with iodine in THF (0.38 M, 57\%).

\section{Preparation of 4-methoxybenzylzinc(II) chloride (1e)}

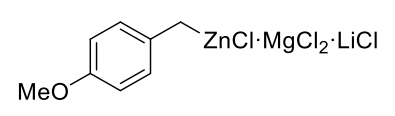

According to TP1, $\mathrm{LiCl}$ ( $0.53 \mathrm{~g}, 12.5 \mathrm{mmol}, 1.25$ equiv), $\mathrm{ZnCl}_{2}$ (1.51 g, $11.0 \mathrm{mmol}, 1.10$ equiv), $\mathrm{Mg}$ turnings ( $0.58 \mathrm{~g}, 24.0 \mathrm{mmol}, 2.40$ equiv) and freshly distilled THF (10 mL) were used. 4-Methoxybenzyl chloride ( $1.57 \mathrm{~g}, 1.36 \mathrm{~mL}, 10.0 \mathrm{mmol}, 1.00$ equiv) was dropwise added and the reaction mixture 
was stirred for $1 \mathrm{~h}$ at room temperature. The concentration of 4-methoxybenzylzinc(II) chloride (1e) was determined by titration with iodine in THF $(0.60 \mathrm{M}, 60 \%)$.

\section{Preparation of 4-(methylthio)benzylzinc(II) chloride (1f)}

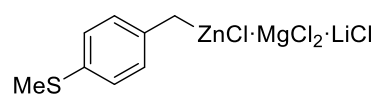

According to TP1, $\mathrm{LiCl}$ (0.53 g, $12.5 \mathrm{mmol}, 1.25$ equiv), $\mathrm{ZnCl}_{2}$ (1.51 g, $11.0 \mathrm{mmol}, 1.10$ equiv), $\mathrm{Mg}$ turnings ( $0.58 \mathrm{~g}, 24.0 \mathrm{mmol}, 2.40$ equiv) and freshly distilled THF $(15 \mathrm{~mL})$ were used. 4-(Methylthio)benzyl chloride (1.73 g, $1.48 \mathrm{~mL}, 10.0 \mathrm{mmol}, 1.00$ equiv) was dropwise added and the reaction mixture was stirred for $1 \mathrm{~h}$ at room temperature. The concentration of 4-(methylthio)benzylzinc(II) chloride (1f) was determined by titration with iodine in THF $(0.35 \mathrm{M}, 53 \%)$.

\section{Preparation of (1-phenylethyl)zinc(II) chloride (1g)}

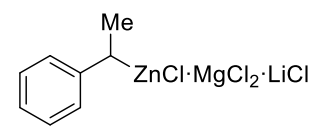

According to TP1, $\mathrm{LiCl}\left(0.53 \mathrm{~g}, 12.5 \mathrm{mmol}, 1.25\right.$ equiv), $\mathrm{ZnCl}_{2}(1.51 \mathrm{~g}, 11.0 \mathrm{mmol}, 1.10$ equiv), $\mathrm{Mg}$ turnings (0.58 g, $24.0 \mathrm{mmol}, 2.40$ equiv) and freshly distilled THF $(10 \mathrm{~mL})$ were used. (1Chloroethyl)benzene ( $1.41 \mathrm{~g}, 1.33 \mathrm{~mL}, 10.0 \mathrm{mmol}, 1.00$ equiv) was dropwise added and the reaction mixture was stirred for $2 \mathrm{~h}$ at room temperature. The concentration of (1-phenylethyl)zinc(II) chloride (1g) was determined by titration with iodine in THF $(0.56 \mathrm{M}, 56 \%)$.

\section{Preparation of the Arylzinc(II) Chlorides (4a-f)}

\section{Preparation of (4-(ethoxycarbonyl)phenyl)zinc(II) chloride (4a)}<smiles>CCOC(=O)c1ccc(C(=O)OCCl)cc1</smiles>

According to TP2, ethyl 4-iodobenzoate ( $2.76 \mathrm{~g}, 1.68 \mathrm{~mL}, 10.0 \mathrm{mmol}, 1.00$ equiv), tetradecane ( $500 \mu \mathrm{L})$ and freshly distilled THF $(10 \mathrm{~mL})$ were used. The solution was cooled to $-20{ }^{\circ} \mathrm{C}$ and $i \mathrm{PrMgCl} \cdot \mathrm{LiCl}$ (10.0 mL, $12.0 \mathrm{mmol}, 1.21 \mathrm{M}$ in THF, 1.20 equiv) was dropwise added. The reaction mixture was stirred for $0.5 \mathrm{~h}$ at the prior adjusted temperature and subsequently transmetalated with $\mathrm{ZnCl}_{2}(12.0 \mathrm{~mL}$, $12.0 \mathrm{mmol}, 1.0 \mathrm{M}$ in THF, 1.20 equiv). The concentration of (4-(ethoxycarbonyl)phenyl)zinc(II) chloride (4a) was determined by titration with iodine in THF $(0.32 \mathrm{M},>90 \%)$. 


\section{Preparation of (3-(ethoxycarbonyl)phenyl)zinc(II) chloride (4b)}

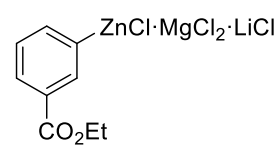

According to TP2, ethyl 3-iodobenzoate ( $2.76 \mathrm{~g}, 1.68 \mathrm{~mL}, 10.0 \mathrm{mmol}, 1.00$ equiv), tetradecane ( $500 \mu \mathrm{L})$ and freshly distilled THF $(10 \mathrm{~mL})$ were used. The solution was cooled to $-20{ }^{\circ} \mathrm{C}$ and $i \mathrm{PrMgCl} \cdot \mathrm{LiCl}$ (10.0 mL, $12.0 \mathrm{mmol}, 1.21 \mathrm{M}$ in THF, 1.20 equiv) was dropwise added. The reaction mixture was stirred for $0.5 \mathrm{~h}$ at the prior adjusted temperature and subsequently transmetalated with $\mathrm{ZnCl}_{2}(12.0 \mathrm{~mL}$, $12.0 \mathrm{mmol}, 1.0 \mathrm{M}$ in THF, 1.20 equiv). The concentration of (3-(ethoxycarbonyl)phenyl)zinc(II) chloride (4b) was determined by titration with iodine in THF $(0.31 \mathrm{M},>90 \%)$.

Preparation of (2-(ethoxycarbonyl)phenyl)zinc(II) chloride (4c)

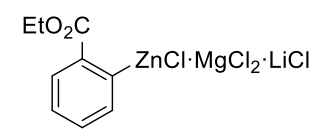

According to TP2, ethyl 2-iodobenzoate ( $2.76 \mathrm{~g}, 1.68 \mathrm{~mL}, 10.0 \mathrm{mmol}, 1.00$ equiv), tetradecane ( $500 \mu \mathrm{L})$ and freshly distilled THF $(10 \mathrm{~mL})$ were used. The solution was cooled to $-20{ }^{\circ} \mathrm{C}$ and $i \mathrm{PrMgCl} \cdot \mathrm{LiCl}$ (10.0 mL, $12.0 \mathrm{mmol}, 1.21 \mathrm{M}$ in THF, 1.20 equiv) was dropwise added. The reaction mixture was stirred for $0.5 \mathrm{~h}$ at the prior adjusted temperature and subsequently transmetalated with $\mathrm{ZnCl}_{2}(12.0 \mathrm{~mL}$, $12.0 \mathrm{mmol}, 1.0 \mathrm{M}$ in THF, 1.20 equiv). The concentration of (2-(ethoxycarbonyl)phenyl)zinc(II) chloride (4c) was determined by titration with iodine in THF $(0.34 \mathrm{M},>90 \%)$.

Preparation of (4-(trifluoromethyl)phenyl)zinc(II) chloride (4d)

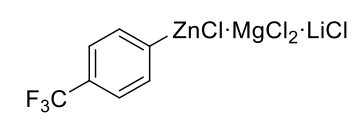

According to TP2, 4-iodobenzotrifluoride $(2.72 \mathrm{~g}, 1.47 \mathrm{~mL}, 10.0 \mathrm{mmol}, 1.00$ equiv), tetradecane $(250 \mu \mathrm{L})$ and freshly distilled THF $(5.0 \mathrm{~mL})$ were used. The solution was cooled to $-20{ }^{\circ} \mathrm{C}$ and $i \mathrm{PrMgCl} \cdot \mathrm{LiCl}(10.0 \mathrm{~mL}, 12.0 \mathrm{mmol}, 1.21 \mathrm{M}$ in THF, 1.20 equiv) was dropwise added. The reaction mixture was stirred for $0.5 \mathrm{~h}$ at the prior adjusted temperature and subsequently transmetalated with $\mathrm{ZnCl}_{2}$ (12.0 mL, $12.0 \mathrm{mmol}, 1.0 \mathrm{M}$ in THF, 1.20 equiv). The concentration of (4-(trifluoromethyl)phenyl)zinc(II) chloride (4d) was determined by titration with iodine in THF $(0.42 \mathrm{M},>90 \%)$.

\section{Preparation of (4-fluoro-3-methylphenyl)zinc(II) chloride (4e)}

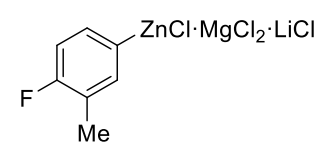

According to TP2, 2-fluoro-5-iodotoluene (2.36 g, $10.0 \mathrm{mmol}, 1.00$ equiv), tetradecane (500 $\mu \mathrm{L}$ ) and freshly distilled THF $(10 \mathrm{~mL})$ were used. The solution was cooled to $-20^{\circ} \mathrm{C}$ and $i \mathrm{PrMgCl} \cdot \mathrm{LiCl}$ (10.0 mL, $12.0 \mathrm{mmol}, 1.21 \mathrm{M}$ in THF, 1.20 equiv) was dropwise added. The reaction mixture was stirred for $0.5 \mathrm{~h}$ at the prior adjusted temperature and subsequently transmetalated with $\mathrm{ZnCl}_{2}(12.0 \mathrm{~mL}$, 
$12.0 \mathrm{mmol}, 1.0 \mathrm{M}$ in THF, 1.20 equiv). The concentration of (4-fluoro-3-methylphenyl)zinc(II) chloride (4e) was determined by titration with iodine in THF $(0.33 \mathrm{M},>90 \%)$

\section{Preparation of (4-methoxyphenyl)zinc(II) chloride (4f)}

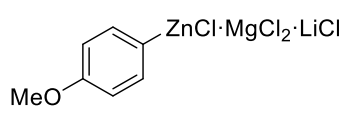

According to TP2, 4-iodoanisole (2.34 g, $10.0 \mathrm{mmol}, 1.00$ equiv), tetradecane (500 $\mu \mathrm{L})$ and freshly distilled THF $(10 \mathrm{~mL})$ were used. The solution was cooled to $-20{ }^{\circ} \mathrm{C}$ and $i \operatorname{PrMgCl} \cdot \mathrm{LiCl}(10.0 \mathrm{~mL}, 12.0$ mmol, $1.21 \mathrm{M}$ in THF, 1.20 equiv) was dropwise added. The reaction mixture was stirred for $0.5 \mathrm{~h}$ at the prior adjusted temperature and subsequently transmetalated with $\mathrm{ZnCl}_{2}(12.0 \mathrm{~mL}, 12.0 \mathrm{mmol}, 1.0 \mathrm{M}$ in THF, 1.20 equiv). The concentration of (4-methoxyphenyl)zinc(II) chloride (4f) was determined by titration with iodine in THF $(0.36 \mathrm{M},>90 \%)$.

\section{Iron-Catalyzed Acylation of Benzylic Zinc Chlorides (1a-g) with Acid Chlorides}

\section{Preparation of 1-(4-chlorophenyl)-2-phenylethan-1-one (3a)}

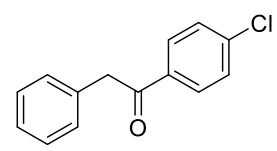

Based on TP 3, $\mathrm{FeCl}_{2}(6.34 \mathrm{mg}, 0.05 \mathrm{mmol}, 5.0 \mathrm{~mol} \%$ ), 4-chlorobenzoyl chloride (2a, $175 \mathrm{mg}, 0.13$ $\mathrm{mL}, 1.00 \mathrm{mmol}, 1.00$ equiv) and freshly distilled THF $(1.0 \mathrm{~mL})$ were used. The benzylzinc(II) chloride solution (1a, $1.56 \mathrm{~mL}, 1.25 \mathrm{mmol}, 0.80 \mathrm{M}$ in THF, 1.25 equiv) was added dropwise and the reaction mixture was stirred for $0.5 \mathrm{~h}$ at room temperature. The reaction was quenched with a saturated aqueous $\mathrm{NH}_{4} \mathrm{Cl}$ solution and the aqueous layer was extracted with EtOAc $(3 \times 75 \mathrm{~mL})$. The combined organic layers were dried over $\mathrm{MgSO}_{4}$, filtered and concentrated under reduced pressure. Purification of the crude product by flash column chromatography $\left(\mathrm{SiO}_{2}, i\right.$-hexane $:$ EtOAc $\left.=19: 1, \mathrm{R}_{\mathrm{f}}=0.45\right)$ afforded the desired product $\mathbf{3 a}$ (208 $\mathrm{mg}, 0.90 \mathrm{mmol}, 90 \%)$ as a white solid.

M.p.: $80.2^{\circ} \mathrm{C}$

${ }^{1} \mathrm{H}-\mathrm{NMR}\left(600 \mathrm{MHz}, \mathbf{C D C l}_{3}, \mathbf{p p m}\right) \delta=7.93(\mathrm{~d}, J=8.5 \mathrm{~Hz}, 2 \mathrm{H}), 7.41(\mathrm{~d}, J=8.8 \mathrm{~Hz}, 2 \mathrm{H}), 7.33-7.31(\mathrm{~m}$, 2H), 7.26-7.23 (m, 3H), 4.24 (s, 2H).

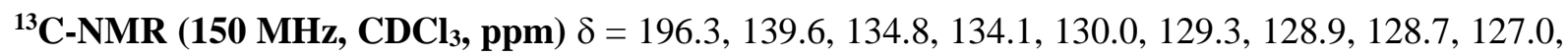
45.5.

IR $\left(A T R, \mathbf{c m}^{-1}\right) \tilde{v}=3028,2901,1723,1686,1588,1571,1496,1485,1452,1395,1336,1320,1271$, 1217, 1201, 1169, 1104, 1088, 1072, 1004, 991, 961, 824, 795, 749, 711, 694, 661. 
MS (EI, 70 eV, \%) m/z = 230 (10), 141 (30), 139 (100), 111 (19), 91 (14), 57 (20), 43 (26), 41 (11)

HRMS (EI, $70 \mathbf{~ e V ) ~ m / z : ~ c a l c . ~ f o r ~} \mathbf{C}_{\mathbf{1 4}} \mathbf{H}_{\mathbf{1 1}}$ ClO: 230.0498; found 230.0484 .

\section{Preparation of 1-(4-(tert-butyl)phenyl)-2-(3-(trifluoromethyl)phenyl)ethan-1-one (3b)}

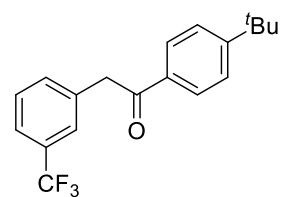

According to $\mathbf{T P 3}, \mathrm{FeCl}_{2}(6.34 \mathrm{mg}, 0.05 \mathrm{mmol}, 5.0 \mathrm{~mol} \%)$, 4-tert-butylbenzoyl chloride (2b, $197 \mathrm{mg}$, $0.20 \mathrm{~mL}, 1.00 \mathrm{mmol}, 1.00$ equiv) and freshly distilled THF (1.0 mL) were used. The 3-(trifluoromethyl)benzylzinc(II) chloride solution (1b, $1.74 \mathrm{~mL}, 1.25 \mathrm{mmol}, 0.72 \mathrm{M}$ in THF, 1.25 equiv) was added dropwise and the reaction mixture was stirred for $0.5 \mathrm{~h}$ at room temperature. The reaction was quenched with a saturated aqueous $\mathrm{NH}_{4} \mathrm{Cl}$ solution and the aqueous layer was extracted with EtOAc $(3 \times 75 \mathrm{~mL})$. The combined organic layers were dried over $\mathrm{MgSO}_{4}$, filtered and concentrated under reduced pressure. The crude product was purified by flash column chromatography $\left(\mathrm{SiO}_{2}, i\right.$-hexane $:$ EtOAc $=99: 1, \mathrm{R}_{\mathrm{f}}$ $=0.15$ ) leading to the desired product $3 \mathbf{b}$ ( $208 \mathrm{mg}, 0.65 \mathrm{mmol}, 65 \%)$ (38\% isolated yield without catalyst after prolonged reaction time of $2 \mathrm{~h}$ at room temperature) as a white solid.

M.p.: $68.3^{\circ} \mathrm{C}$

${ }^{1} \mathrm{H}-\mathrm{NMR}\left(400 \mathrm{MHz}, \mathbf{C D C l}_{3}, \mathbf{p p m}\right) \delta=7.96(\mathrm{~d}, J=8.7 \mathrm{~Hz}, 2 \mathrm{H}), 7.56-7.43(\mathrm{~m}, 6 \mathrm{H}), 4.33(\mathrm{~s}, 2 \mathrm{H}), 1.35$ $(\mathrm{s}, 9 \mathrm{H})$.

${ }^{13} \mathbf{C}-N M R\left(100 ~ M H z, C^{2} C_{3}, \mathbf{p p m}\right) \delta=196.1,157.2,135.4,133.6,132.9\left(\mathrm{q},{ }^{3} J(\mathrm{C}, \mathrm{F})=4.0 \mathrm{~Hz}\right), 130.7$ $\left(\mathrm{q},{ }^{2} J(\mathrm{C}, \mathrm{F})=32 \mathrm{~Hz}\right), 128.8,128.3,126.2\left(\mathrm{q},{ }^{3} J(\mathrm{C}, \mathrm{F})=3.8 \mathrm{~Hz}\right), 125.6,123.9\left(\mathrm{q},{ }^{1} J(\mathrm{C}, \mathrm{F})=271 \mathrm{~Hz}\right), 123.6$ $\left(\mathrm{q},{ }^{3} \mathrm{~J}(\mathrm{C}, \mathrm{F})=3.8 \mathrm{~Hz}\right), 44.7,35.0,30.9$.

${ }^{19}$ F-NMR (376 MHz, $\left.\mathrm{CDCl}_{3}, \mathrm{ppm}\right) \delta=-62.6$.

IR $\left(\mathbf{A T R}, \mathbf{c m}^{-1}\right) \tilde{\mathrm{v}}=2964,1725,1711,1678,1641,1603,1451,1406,1366,1331,1268,1223,1160$, 1110, 1092, 1075, 1004, 992, 915, 880, 841, 829, 786, 720, 701, 658.

MS (EI, 70 eV, \%) m/z = 320 (1), 305 (10), 162 (12), 161 (100), 118 (10).

HRMS (EI, $70 \mathbf{~ e V ) ~ m / z : ~ c a l c . ~ f o r ~} \mathbf{C}_{19} \mathbf{H}_{19} \mathbf{F}_{3} \mathbf{O}$ : 320.1388; found 320.1386 . 


\section{Preparation of 1-(4-chlorophenyl)-2-(3-(trifluoromethyl)phenyl)ethan-1-one (3c)}

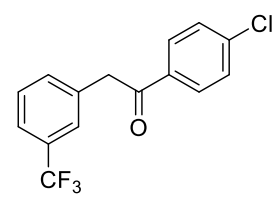

According to TP3, $\mathrm{FeCl}_{2}(6.34 \mathrm{mg}, 0.05 \mathrm{mmol}, 5.0 \mathrm{~mol} \%$ ), 4-chlorobenzoyl chloride (2a, $175 \mathrm{mg}, 0.13$ $\mathrm{mL}, 1.00 \mathrm{mmol}, 1.00$ equiv) and freshly distilled THF $(1.0 \mathrm{~mL})$ were used. The 3-(trifluoromethyl)benzylzinc(II) chloride solution (1b, $1.74 \mathrm{~mL}, 1.25 \mathrm{mmol}, 0.72 \mathrm{M}$ in THF, 1.25 equiv) was added dropwise and the reaction mixture was stirred for $0.5 \mathrm{~h}$ at room temperature. The reaction was quenched with a saturated aqueous $\mathrm{NH}_{4} \mathrm{Cl}$ solution and the aqueous layer was extracted with EtOAc $(3 \times 75 \mathrm{~mL})$. The combined organic layers were dried over $\mathrm{MgSO}_{4}$, filtered and concentrated under reduced pressure. Purification of the crude product by flash column chromatography $\left(\mathrm{SiO}_{2}, i\right.$-hexane $:$ EtOAc $=19: 1, \mathrm{R}_{\mathrm{f}}$ $=0.22)$ furnished the product $3 \mathbf{c}(212 \mathrm{mg}, 0.71 \mathrm{mmol}, 71 \%)$ as a white solid.

M.p.: $54.5^{\circ} \mathrm{C}$

${ }^{1} \mathrm{H}-\mathrm{NMR}\left(400 \mathrm{MHz}, \mathbf{C D C l}_{3}, \mathbf{p p m}\right) \delta=7.95(\mathrm{~d}, J=8.7 \mathrm{~Hz}, 2 \mathrm{H}), 7.57-7.39(\mathrm{~m}, 6 \mathrm{H}), 4.32(\mathrm{~s}, 2 \mathrm{H})$.

${ }^{13}$ C-NMR (100 MHz, CDCl 3 , ppm) $\delta=195.8,140.5,135.5,135.1,133.5\left(\mathrm{q},{ }^{4} J(\mathrm{C}, \mathrm{F})=2.0 \mathrm{~Hz}\right), 131.4$ $\left(\mathrm{q},{ }^{2} J(\mathrm{C}, \mathrm{F})=32 \mathrm{~Hz}\right), 130.3,129.6,129.5,126.8\left(\mathrm{q},{ }^{3} J(\mathrm{C}, \mathrm{F})=3.8 \mathrm{~Hz}\right), 124.5\left(\mathrm{q},{ }^{1} J(\mathrm{C}, \mathrm{F})=270 \mathrm{~Hz}\right), 124.4$ $\left(\mathrm{q},{ }^{3} \mathrm{~J}(\mathrm{C}, \mathrm{F})=3.8 \mathrm{~Hz}\right), 45.3$.

${ }^{19}$ F-NMR (376 MHz, $\left.\mathrm{CDCl}_{3}, \mathrm{ppm}\right) \delta=-62.6$.

IR $\left(A T R, \mathbf{c m}^{-1}\right) \tilde{v}=2916,1726,1694,1681,1587,1570,1488,1454,1400,1330,1209,1185,1156$, 1120, 1092, 1074, 1013, 1003, 990, 902, 878, 848, 812, 784, 700, 676, 657.

MS (EI, 70 eV, \%) m/z = 298 (1), 141 (31), 140 (10), 139 (100), 111 (22), 75 (10).

HRMS (EI, $70 \mathbf{e V}$ ) m/z: calc. for $\mathbf{C}_{15} \mathbf{H}_{10} \mathbf{C l F}_{3} \mathbf{O}$ : 298.0372; found 298.0359.

Preparation of 1-(4-(tert-butyl)phenyl)-2-(3-fluorophenyl)ethan-1-one (3d)

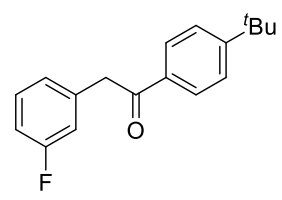

Based on TP3, $\mathrm{FeCl}_{2}$ (6.34 mg, $0.05 \mathrm{mmol}, 5.0 \mathrm{~mol} \%$ ), 4-tert-butylbenzoyl chloride (2b, $197 \mathrm{mg}$, $0.20 \mathrm{~mL}, 1.00 \mathrm{mmol}, 1.00$ equiv) and freshly distilled THF $(1.0 \mathrm{~mL})$ were used. The 3-fluoro- 
benzylzinc(II) chloride (1c, $1.92 \mathrm{~mL}, 1.25 \mathrm{mmol}, 0.65 \mathrm{M}$ in THF, 1.25 equiv) was added dropwise and the reaction mixture was stirred for $0.5 \mathrm{~h}$ at room temperature. The reaction was quenched with a saturated aqueous $\mathrm{NH}_{4} \mathrm{Cl}$ solution and the aqueous layer was extracted with EtOAc $(3 \times 75 \mathrm{~mL})$. The combined organic layers were dried over $\mathrm{MgSO}_{4}$, filtered and concentrated under reduced pressure. The crude product was purified by flash column chromatography $\left(\mathrm{SiO}_{2}, i\right.$-hexane $:$ EtOAc $=19: 1, \mathrm{R}_{\mathrm{f}}=$ $0.50)$ leading to the desired product $\mathbf{3 d}(237 \mathrm{mg}, 0.88 \mathrm{mmol}, 88 \%)$ as a pale yellow oil.

${ }^{1} \mathrm{H}-\mathrm{NMR}\left(\mathbf{4 0 0} \mathrm{MHz}, \mathbf{C D C l}_{3}\right.$, ppm) $\delta=7.91(\mathrm{~d}, J=8.7 \mathrm{~Hz}, 2 \mathrm{H}), 7.44(\mathrm{~d}, J=8.7 \mathrm{~Hz}, 2 \mathrm{H}), 7.27-7.18(\mathrm{~m}$, 1H), 7.02-6.85 (m, 3H), 4.21 (s, 2H), 1.29 (s, 9H).

${ }^{13} \mathrm{C}-\mathrm{NMR}\left(100 \mathrm{MHz}, \mathbf{C D C l}_{3}\right.$, ppm) $\delta=196.3,162.7\left(\mathrm{~d},{ }^{1} J(\mathrm{C}, \mathrm{F})=245 \mathrm{~Hz}\right), 157.0,137.0\left(\mathrm{~d},{ }^{3} J(\mathrm{C}, \mathrm{F})=\right.$ $7.7 \mathrm{~Hz}), 133.7,129.8\left(\mathrm{~d},{ }^{3} J(\mathrm{C}, \mathrm{F})=8.4 \mathrm{~Hz}\right), 128.4,125.5,125.0\left(\mathrm{~d},{ }^{4} J(\mathrm{C}, \mathrm{F})=2.9 \mathrm{~Hz}\right), 116.3\left(\mathrm{~d},{ }^{2} J(\mathrm{C}, \mathrm{F})\right.$ $=22 \mathrm{~Hz}), 113.6\left(\mathrm{~d},{ }^{2} J(\mathrm{C}, \mathrm{F})=21 \mathrm{~Hz}\right), 44.8,35.0,30.9$.

${ }^{19}$ F-NMR (376 MHz, $\left.\mathrm{CDCl}_{3}, \mathrm{ppm}\right) \delta=-113.1$.

IR $\left(A T R, \mathbf{c m}^{-1}\right) \tilde{v}=3062,2964,2906,2870,1679,1604,1590,1488,1449,1406,1364,1329,1299$, $1268,1254,1218,1192,1141,1108,994,948,850,828,774,754,711,681$.

MS (EI, 70 eV, \%) m/z = 272 (1), 272 (3), 162 (10), 161 (55), 70 (11), 61 (16), 45 (15), 43 (100).

HRMS (EI, 70 eV) m/z: calc. for $\mathbf{C}_{18} \mathbf{H}_{19} \mathbf{F O}$ : 270.1420; found 270.1413 .

\section{Preparation of 2-(3-fluorophenyl)-1-(4-fluorophenyl)ethan-1-one (3e)}

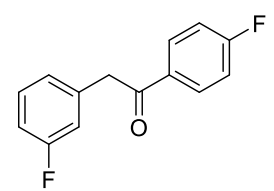

According to TP3, $\mathrm{FeCl}_{2}$ (6.34 mg, $0.05 \mathrm{mmol}, 5.0 \mathrm{~mol} \%$ ), 4-fluorobenzoyl chloride (2c, $159 \mathrm{mg}, 0.12$ $\mathrm{mL}, 1.00 \mathrm{mmol}, 1.00$ equiv) and freshly distilled THF $(1.0 \mathrm{~mL})$ were used. The 3-fluorobenzylzinc(II) chloride (1c, $1.92 \mathrm{~mL}, 1.25 \mathrm{mmol}, 0.65 \mathrm{M}$ in THF, 1.25 equiv) was added dropwise and the reaction mixture was stirred for $0.5 \mathrm{~h}$ at room temperature. The reaction was quenched with a saturated aqueous $\mathrm{NH}_{4} \mathrm{Cl}$ solution and the aqueous layer was extracted with EtOAc $(3 \times 75 \mathrm{~mL})$. The combined organic layers were dried over $\mathrm{MgSO}_{4}$, filtered and concentrated under reduced pressure. Purification of the crude product by flash column chromatography $\left(\mathrm{SiO}_{2}, i\right.$-hexane $:$ EtOAc $\left.=19: 1, \mathrm{R}_{\mathrm{f}}=0.38\right)$ afforded the product $3 \mathbf{e}$ (172 $\mathrm{mg}, 0.74 \mathrm{mmol}, 74 \%)$ as a colourless oil. 
M.p.: $48.6^{\circ} \mathrm{C}$

${ }^{1} \mathbf{H}-\mathbf{N M R}\left(400 \mathrm{MHz}, \mathbf{C D C l}_{3}, \mathbf{p p m}\right) \delta=7.96(\mathrm{dd}, J=9.0,5.4 \mathrm{~Hz}, 2 \mathrm{H}), 7.26-7.18(\mathrm{~m}, 1 \mathrm{H})$, 7.10-7.03 (m, 2H), 6.99-6.85 (m, 3H), 4.18 (s, 2H).

${ }^{13} \mathrm{C}-\mathrm{NMR}\left(100 \mathrm{MHz}, \mathrm{CDCl}_{3}\right.$, ppm) $\delta=195.4,166.0\left(\mathrm{~d},{ }^{1} \mathrm{~J}(\mathrm{C}, \mathrm{F})=254 \mathrm{~Hz}\right), 163.0\left(\mathrm{~d},{ }^{1} \mathrm{~J}(\mathrm{C}, \mathrm{F})=245\right.$ $\mathrm{Hz}), 136.8\left(\mathrm{~d},{ }^{3} J(\mathrm{C}, \mathrm{F})=7.7 \mathrm{~Hz}\right), 132.9\left(\mathrm{~d},{ }^{4} \mathrm{~J}(\mathrm{C}, \mathrm{F})=3.0 \mathrm{~Hz}\right), 131.3\left(\mathrm{~d},{ }^{3} J(\mathrm{C}, \mathrm{F})=9.4 \mathrm{~Hz}\right), 130.3(\mathrm{~d}$, $\left.{ }^{3} J(\mathrm{C}, \mathrm{F})=8.4 \mathrm{~Hz}\right), 125.3\left(\mathrm{~d},{ }^{4} J(\mathrm{C}, \mathrm{F})=2.9 \mathrm{~Hz}\right), 116.6\left(\mathrm{~d},{ }^{2} J(\mathrm{C}, \mathrm{F})=22 \mathrm{~Hz}\right), 116.0\left(\mathrm{~d},{ }^{2} J(\mathrm{C}, \mathrm{F})=22 \mathrm{~Hz}\right)$, $114.1\left(\mathrm{~d},{ }^{2} \mathrm{~J}(\mathrm{C}, \mathrm{F})=21 \mathrm{~Hz}\right), 45.1$.

${ }^{19}$ F-NMR (376 MHz, $\left.\mathrm{CDCl}_{3}, \mathbf{p p m}\right) \delta=-104.6,-112.9$.

IR $\left(\mathbf{A T R}, \mathbf{c m}^{-1}\right) \tilde{\mathrm{v}}=3061,2927,2854,1680,1591,1507,1488,1448,1412,1327,1298,1250,1236$, 1207, 1156, 1144, 1100, 1008, 994, 954, 941, 886, 831, 815, 769, 738, 684.

MS (EI, 70 eV, \%) m/z = 233 (8), 232 (1), 124 (10), 123 (100), 95 (28), 70 (10), 61 (13), 45 (11), 42 (79).

HRMS (EI, 70 eV) m/z: calc. for $\mathbf{C}_{\mathbf{1 4}} \mathbf{H}_{\mathbf{1 0}} \mathbf{F}_{\mathbf{2}} \mathbf{O}$ : 232.0700; found 232.0694.

Preparation of ethyl 3-(2-(4-chlorophenyl)-2-oxoethyl)benzoate (3f)

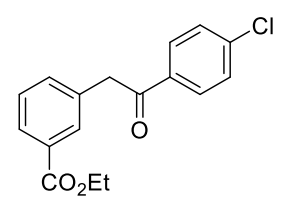

Based on TP3, $\mathrm{FeCl}_{2}(6.34 \mathrm{mg}, 0.05 \mathrm{mmol}, 5.0 \mathrm{~mol} \%)$, 4-chlorobenzoyl chloride (2a, $175 \mathrm{mg}, 0.13$ $\mathrm{mL}, 1.00 \mathrm{mmol}, 1.00$ equiv) and freshly distilled THF (1.0 mL) were used. The 3-(ethoxycarbonyl)benzylzinc(II) chloride (1d, $3.30 \mathrm{~mL}, 1.25 \mathrm{mmol}, 0.38 \mathrm{M}$ in THF, 1.25 equiv) was added dropwise and the reaction mixture was stirred for $4 \mathrm{~h}$ at room temperature. The reaction was quenched with a saturated aqueous $\mathrm{NH}_{4} \mathrm{Cl}$ solution and the aqueous layer was extracted with EtOAc $(3 \times 75 \mathrm{~mL})$. The combined organic layers were dried over $\mathrm{MgSO}_{4}$, filtered and concentrated under reduced pressure. The crude product was purified by flash column chromatography $\left(\mathrm{SiO}_{2}, i\right.$-hexane $:$ EtOAc $\left.=9: 1, \mathrm{R}_{\mathrm{f}}=0.21\right)$ leading to the product $\mathbf{3 f}(151 \mathrm{mg}, 0.50 \mathrm{mmol}, 50 \%)$ as pale yellow solid. 
M.p.: $77.1^{\circ} \mathrm{C}$.

${ }^{1} \mathbf{H}-\mathbf{N M R}\left(\mathbf{6 0 0} \mathbf{M H z}, \mathbf{C D C l}_{3}, \mathbf{p p m}\right) \delta=7.94-7.92(\mathrm{~m}, 4 \mathrm{H}), 7.44-7.38(\mathrm{~m}, 4 \mathrm{H}), 4.36(\mathrm{q}, J=7.1 \mathrm{~Hz}, 2 \mathrm{H})$, $4.30(\mathrm{~s}, 2 \mathrm{H}), 1.37(\mathrm{t}, J=7.1 \mathrm{~Hz}, 3 \mathrm{H})$.

${ }^{13} \mathbf{C}-N M R\left(150 ~ M H z, \mathbf{C D C l}_{3}, \mathbf{p p m}\right) \delta=195.8,166.3,139.8,134.7,134.4,133.9,130.9,129.9,129.0$, $128.7,128.3,61.0,45.1,14.3$.

IR $\left(A T R, \mathbf{c m}^{-1}\right) \tilde{v}=2982,2915,2853,1703,1693,1606,1587,1570,1483,1444,1396,1365,1332$, 1280, 1170, 1106, 1087, 1029, 1000, 990, 831, 815, 796, 755, 721, 672.

MS (EI, 70 eV, \%) m/z = 302 (5), 141 (30), 139 (100), 111 (15).

HRMS (EI, $70 \mathrm{eV}$ ) m/z: calc. for $\mathbf{C}_{17} \mathbf{H}_{15} \mathrm{ClO}_{3}$ : 302.0710; found 302.0698 .

Preparation of 1-(4-(tert-butyl)phenyl)-2-(4-methoxyphenyl)ethan-1-one (3g)

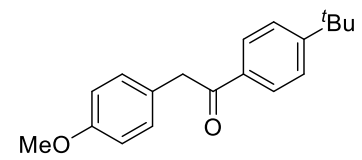

According to TP3, $\mathrm{FeCl}_{2}(6.34 \mathrm{mg}, 0.05 \mathrm{mmol}, 5.0 \mathrm{~mol} \%)$, 4-tert-butylbenzoyl chloride (2b, $197 \mathrm{mg}$, $0.20 \mathrm{~mL}, 1.00 \mathrm{mmol}, 1.00$ equiv) and freshly distilled THF (1.0 mL) were used. The 4-methoxybenzylzinc(II) chloride (1e, $2.10 \mathrm{~mL}, 1.25 \mathrm{mmol}, 0.60 \mathrm{M}$ in THF, 1.25 equiv) was added dropwise and the reaction mixture was stirred for $0.5 \mathrm{~h}$ at room temperature. The reaction was quenched with a saturated aqueous $\mathrm{NH}_{4} \mathrm{Cl}$ solution and the aqueous layer was extracted with EtOAc $(3 \times 75 \mathrm{~mL})$. The combined organic layers were dried over $\mathrm{MgSO}_{4}$, filtered and concentrated under reduced pressure. Purification of the crude product by flash column chromatography $\left(\mathrm{SiO}_{2}, i\right.$-hexane $:$ EtOAc $=19: 1, \mathrm{R}_{\mathrm{f}}$ $=0.28)$ gave the product $3 \mathrm{~g}(164 \mathrm{mg}, 0.58 \mathrm{mmol}, 58 \%)$ as a pale yellow solid.

M.p.: $109.7^{\circ} \mathrm{C}$

${ }^{1} \mathrm{H}-\mathrm{NMR}\left(400 \mathrm{MHz}, \mathbf{C D C l}_{3}\right.$, ppm) $\delta=7.89(\mathrm{~d}, J=6.7 \mathrm{~Hz}, 2 \mathrm{H}), 7.40(\mathrm{~d}, J=6.7 \mathrm{~Hz}, 2 \mathrm{H}), 7.12(\mathrm{~d}, J=$ $8.7 \mathrm{~Hz}, 2 \mathrm{H}), 6.80(\mathrm{~d}, J=8.7 \mathrm{~Hz}, 2 \mathrm{H}), 4.13(\mathrm{~s}, 2 \mathrm{H}), 3.71(\mathrm{~s}, 3 \mathrm{H}), 1.27(\mathrm{~s}, 9 \mathrm{H})$.

${ }^{13}$ C-NMR (100 MHz, $\left.\mathbf{C D C l}_{3}, \mathbf{p p m}\right) \delta=197.5,158.5,156.8,134.0,130.4,128.6,126.8,125.6,114.1$, $55.3,44.6,35.1,31.1$. 
IR $\left(\mathbf{A T R}, \mathbf{c m}^{-1}\right) \tilde{\mathrm{v}}=2952,2901,2869,2829,1721,1683,1601,1511,1462,1404,1360,1332(\mathrm{w})$, 1300, 1268, 1241, 1174, 1104, 1040, 1022, 994, 860, 824, 794, 692.

MS (EI, 70 eV, \%) m/z = 283 (1), 282 (5), 163 (12), 161 (100), 120 (17).

HRMS (EI, $70 \mathbf{~ e V ) ~ m / z : ~ c a l c . ~ f o r ~} \mathbf{C}_{19} \mathbf{H}_{22} \mathbf{O}_{2}$ : 282.1620; found 282.1615 .

\section{Preparation of 2-(4-(methylthio)phenyl)-1-(thiophen-2-yl)ethan-1-one (3h)}

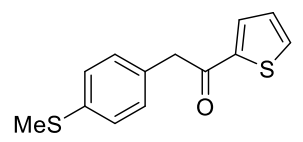

Based on TP3, $\mathrm{FeCl}_{2}$ (6.34 mg, $0.05 \mathrm{mmol}, 5.0 \mathrm{~mol} \%$ ), 2-thiophenecarbonyl chloride (2d, $147 \mathrm{mg}$, $0.11 \mathrm{~mL}, 1.00 \mathrm{mmol}, 1.00$ equiv) and freshly distilled THF $(1.0 \mathrm{~mL})$ were used. The 4-(methylthio)benzylzinc(II) chloride (1f, $3.60 \mathrm{~mL}, 1.25 \mathrm{mmol}, 0.35 \mathrm{M}$ in THF, 1.25 equiv) was added dropwise and the reaction mixture was stirred for $4 \mathrm{~h}$ at room temperature. The reaction was quenched with a saturated aqueous $\mathrm{NH}_{4} \mathrm{Cl}$ solution and the aqueous layer was extracted with EtOAc $(3 \times 75 \mathrm{~mL})$. The combined organic layers were dried over $\mathrm{MgSO}_{4}$, filtered and concentrated under reduced pressure. The crude product was purified by flash column chromatography $\left(\mathrm{SiO}_{2}, i\right.$-hexane $:$ EtOAc $\left.=19: 1, \mathrm{R}_{\mathrm{f}}=0.35\right)$ leading to the product $\mathbf{3 h}$ (149 $\mathrm{mg}, 0.60 \mathrm{mmol}, 60 \%)$ as pale yellow solid.

M.p.: $106.2{ }^{\circ} \mathrm{C}$

${ }^{1} \mathrm{H}-\mathrm{NMR}\left(400 \mathrm{MHz}, \mathrm{CDCl}_{3}, \mathbf{p p m}\right) \delta=7.69(\mathrm{~d}, J=3.6 \mathrm{~Hz}, 1 \mathrm{H}), 7.56(\mathrm{~d}, J=4.8 \mathrm{~Hz}, 1 \mathrm{H}), 7.16(\mathrm{~s}, 4 \mathrm{H})$, $7.05(\mathrm{t}, J=8.0 \mathrm{~Hz}, 1 \mathrm{H}), 4.08(\mathrm{~s}, 2 \mathrm{H}), 2.39$ (s, 3H).

${ }^{13}$ C-NMR (100 MHz, CDCl $\mathbf{3}$, ppm) $\delta=190.3,143.8,137.2,134.1,132.6,131.1,130.0,128.2,127.0$, $45.8,16.0$.

IR $\left(\mathbf{A T R}, \mathbf{c m}^{-1}\right) \tilde{\mathrm{v}}=3070,2920,1660,1602,1516,1494,1412,1402,1354,1326,1224,1198,1116$, 1084, 1060, 956, 940, 916, 854, 796, 760, 744, 658.

MS (EI, 70 eV, \%) m/z = 248 (14), 138 (12), 137 (100), 122 (12), 111 (48).

HRMS (EI, $70 \mathbf{~ e V ) ~ m / z : ~ c a l c . ~ f o r ~} \mathbf{C}_{13} \mathbf{H}_{12} \mathbf{O S}_{2}$ : 248.0330; found 248.0330 . 


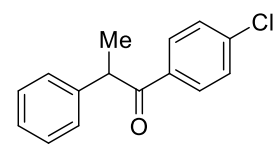

According to $\mathbf{T P 3}, \mathrm{FeCl}_{2}(6.34 \mathrm{mg}, 0.05 \mathrm{mmol}, 5.0 \mathrm{~mol} \%)$, 4-chlorobenzoyl chloride (2a, $175 \mathrm{mg}$, $0.13 \mathrm{~mL}, 1.00 \mathrm{mmol}, 1.00$ equiv) and freshly distilled THF (1.0 mL) were used. The (1-phenylethyl)zinc(II) chloride (1g, $2.25 \mathrm{~mL}, 1.25 \mathrm{mmol}, 0.56 \mathrm{M}$ in THF, 1.25 equiv) was added dropwise and the reaction mixture was stirred for $1 \mathrm{~h}$ at room temperature. The reaction was quenched with a saturated aqueous $\mathrm{NH}_{4} \mathrm{Cl}$ solution and the aqueous layer was extracted with EtOAc $(3 \times 75 \mathrm{~mL})$. The combined organic layers were dried over $\mathrm{MgSO}_{4}$, filtered and concentrated under reduced pressure. Purification of the crude product by flash column chromatography $\left(\mathrm{SiO}_{2}, i\right.$-hexane $:$ EtOAc $\left.=99: 1, \mathrm{R}_{\mathrm{f}}=0.34\right)$ afforded the product $3 \mathbf{i}$ (192 $\mathrm{mg}, 0.78 \mathrm{mmol}, 78 \%$ ) as a colourless oil.

${ }^{1} \mathrm{H}-\mathrm{NMR}\left(400 \mathrm{MHz}, \mathbf{C D C l}_{3}, \mathbf{p p m}\right) \delta=7.81(\mathrm{~d}, J=8.7 \mathrm{~Hz}, 2 \mathrm{H}), 7.27(\mathrm{~d}, J=8.6 \mathrm{~Hz}, 2 \mathrm{H}), 7.25-7.10(\mathrm{~m}$, $5 \mathrm{H}), 4.55(\mathrm{q}, J=6.8 \mathrm{~Hz}, 1 \mathrm{H}), 1.47(\mathrm{~d}, J=6.8 \mathrm{~Hz}, 3 \mathrm{H})$.

${ }^{13}$ C-NMR (100 MHz, CDCl $\left.\mathbf{3}, \mathbf{p p m}\right) \delta=199.0,141.2,139.2,134.8,130.2,129.1,128.8,127.7,127.1$, 48.1, 19.4.

IR $\left(A T R, \mathbf{c m}^{-1}\right) \tilde{v}=3062,3028,2975,2931,2871,1681,1587,1569,1490,1451,1400,1373,1332$, 1248, 1217, 1174, 1091, 1063, 1013, 1000, 950, 847, 786, 754, 731, 698.

MS (EI, 70 eV, \%) m/z = 244 (2), 139 (100), 138 (48), 105 (24).

HRMS (EI, $70 \mathbf{~ e V}$ ) m/z: calc. for $\mathbf{C}_{15} \mathbf{H}_{13}$ ClO: 244.0655; found 244.0652 .

\section{Preparation of 2-phenyl-1-(thiophen-2-yl)propan-1-one (3j)}

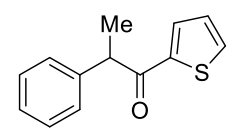

According to TP3, $\mathrm{FeCl}_{2}(6.34 \mathrm{mg}, 0.05 \mathrm{mmol}, 5.0 \mathrm{~mol} \%$ ), 2-thiophenecarbonyl chloride (2d, $147 \mathrm{mg}$, $0.11 \mathrm{~mL}, 1.00 \mathrm{mmol}, 1.00$ equiv) and freshly distilled THF $(1.0 \mathrm{~mL})$ were used. The (1-phenylethyl)zinc(II) chloride (1g, $2.25 \mathrm{~mL}, 1.25 \mathrm{mmol}, 0.56 \mathrm{M}$ in THF, 1.25 equiv) was added dropwise and the reaction mixture was stirred for $1 \mathrm{~h}$ at room temperature. The reaction was quenched with a saturated aqueous $\mathrm{NH}_{4} \mathrm{Cl}$ solution and the aqueous layer was extracted with EtOAc $(3 \times 75 \mathrm{~mL})$. The combined organic layers were dried over $\mathrm{MgSO}_{4}$, filtered and concentrated under reduced pressure. The crude 
product was purified by flash column chromatography $\left(\mathrm{SiO}_{2}, i\right.$-hexane $:$ EtOAc $\left.=19: 1, \mathrm{R}_{\mathrm{f}}=0.45\right)$ leading to the product $\mathbf{3 j}$ (141 $\mathrm{mg}, 0.65 \mathrm{mmol}, 65 \%$ ) as pale yellow oil.

${ }^{1} \mathrm{H}-\mathrm{NMR}\left(400 \mathrm{MHz}, \mathbf{C D C l}_{3}, \mathbf{p p m}\right) \delta=7.61(\mathrm{dd}, J=3.8,1.1 \mathrm{~Hz}, 1 \mathrm{H}), 7.48(\mathrm{dd}, J=4.9,1.1 \mathrm{~Hz}, 1 \mathrm{H})$, 7.30-7.21 (m, 4H), 7.20-7.13 (m, 1H), 6.97 (dd, $J=4.9,3.8 \mathrm{~Hz}, 1 \mathrm{H}), 4.45$ (q, $J=6.9 \mathrm{~Hz}, 1 \mathrm{H}), 1.49$ (d, $J=6.9 \mathrm{~Hz}, 3 \mathrm{H})$.

${ }^{13}$ C-NMR (100 MHz, $\mathbf{C D C l}_{3}$, ppm) $\delta=193.3,143.8,141.3,133.6,132.4,128.9,128.0,127.8,127.1$, 49.4, 19.2.

IR $\left(\mathbf{A T R}, \mathbf{c m}^{-1}\right) \tilde{\mathrm{v}}=3087,3027,2975,2930,2870,1657,1600,1517,1491,1451,1412,1372,1354$, 1253, 1234, 1216, 1182, 1053, 1031, 942, 906, 854, 798, 719, 697.

MS (EI, 70 eV, \%) m/z = 217 (3), 216 (5), 111 (100), 105 (28), 69 (12), 57 (12).

HRMS (EI, $70 \mathbf{~ e V ) ~ m / z : ~ c a l c . ~ f o r ~} \mathbf{C}_{\mathbf{1 3}} \mathbf{H}_{\mathbf{1 2}} \mathbf{O S}$ : 216.0609; found 216.0598 .

Preparation of 1-(4-methoxyphenyl)-2-phenylpropan-1-one (3k)

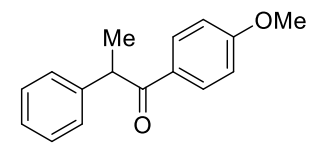

Based on TP3, $\mathrm{FeCl}_{2}(6.34 \mathrm{mg}, 0.05 \mathrm{mmol}, 5.0 \mathrm{~mol} \%)$, 4-methoxybenzoyl chloride (2e, $171 \mathrm{mg}$, $1.00 \mathrm{mmol}, 1.00$ equiv) and freshly distilled THF (1.0 mL) were used. The (1-phenylethyl)-zinc(II) chloride (1g, $2.25 \mathrm{~mL}, 1.25 \mathrm{mmol}, 0.56 \mathrm{M}$ in THF, 1.25 equiv) was added dropwise and the reaction mixture was stirred for $1 \mathrm{~h}$ at room temperature. The reaction was quenched with a saturated aqueous $\mathrm{NH}_{4} \mathrm{Cl}$ solution and the aqueous layer was extracted with EtOAc $(3 \times 75 \mathrm{~mL})$. The combined organic layers were dried over $\mathrm{MgSO}_{4}$, filtered and concentrated under reduced pressure. Purification of the crude product by flash column chromatography $\left(\mathrm{SiO}_{2}, i\right.$-hexane $:$ EtOAc $\left.=19: 1, \mathrm{R}_{\mathrm{f}}=0.28\right)$ furnished the product 3k (190 mg, $0.79 \mathrm{mmol}, 79 \%)$ as a colourless oil.

${ }^{1} \mathrm{H}-\mathrm{NMR}\left(400 \mathrm{MHz}, \mathbf{C D C l}_{3}, \mathbf{p p m}\right) \delta=7.89(\mathrm{~d}, J=9.0 \mathrm{~Hz}, 2 \mathrm{H}), 7.28-7.19(\mathrm{~m}, 4 \mathrm{H}), 6.79(\mathrm{~d}, J=9.0 \mathrm{~Hz}$, 2H), $4.58(\mathrm{q}, J=6.9 \mathrm{~Hz}, 1 \mathrm{H}), 3.73(\mathrm{~s}, 3 \mathrm{H}), 1.46(\mathrm{~d}, J=6.9 \mathrm{~Hz}, 3 \mathrm{H})$.

${ }^{13}$ C-NMR (100 MHz, CDCl $\left.\mathbf{3}, \mathbf{p p m}\right) \delta=198.8,163.2,142.0,131.1,129.5,128.9,127.7,126.8,113.7$, $55.4,47.5,19.6$. 
IR $\left(\mathbf{A T R}, \mathbf{c m}^{-1}\right) \tilde{v}=2972,2931,2840,1710,1671,1598,1573,1510,1493,1452,1418,1372,1333$, 1314, 1248, 1224, 1166, 1115, 1062, 1028, 951, 845, 795, 772, 751, 699.

MS (EI, 70 eV, \%) m/z = 241 (1), 136 (10), 135 (100), 77 (13).

HRMS (EI, $70 \mathbf{~ e V ) ~ m / z : ~ c a l c . ~ f o r ~} \mathbf{C}_{16} \mathbf{H}_{16} \mathbf{O}_{2}$ : 240.1150; found 241.1148 .

\section{Iron-Catalyzed Acylation of Arylzinc Chlorides (4a-f) with Acid Chlorides}

\section{Preparation of ethyl 4-(4-chlorobenzoyl)benzoate (5a)}

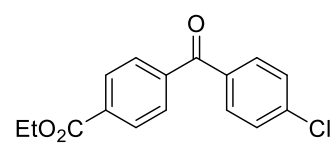

According to TP4, $\mathrm{FeCl}_{2}$ (6.34 mg, $0.05 \mathrm{mmol}, 5.0 \mathrm{~mol} \%$ ), 4-chlorobenzoyl chloride (2a, $175 \mathrm{mg}$, $0.13 \mathrm{~mL}, 1.00 \mathrm{mmol}, 1.00$ equiv) and freshly distilled THF (1.0 mL) were used. The (4-(ethoxycarbonyl)phenyl)zinc(II) chloride (4a, $3.90 \mathrm{~mL}, 1.25 \mathrm{mmol}, 0.32 \mathrm{M}$ in THF, 1.25 equiv) was added dropwise and the reaction mixture was stirred for $4 \mathrm{~h}$ at $50{ }^{\circ} \mathrm{C}$. The reaction was quenched with a saturated aqueous $\mathrm{NH}_{4} \mathrm{Cl}$ solution and the aqueous layer was extracted with EtOAc $(3 \times 75 \mathrm{~mL})$. The combined organic layers were dried over $\mathrm{MgSO}_{4}$, filtered and concentrated under reduced pressure. Purification of the crude product by flash column chromatography $\left(\mathrm{SiO}_{2}, i\right.$-hexane $:$ EtOAc $=99: 1, \mathrm{R}_{\mathrm{f}}$ $=0.35)$ afforded the product $\mathbf{5 a}(179 \mathrm{mg}, 0.62 \mathrm{mmol}, 62 \%)(34 \%$ isolated yield without catalyst after prolonged reaction time of $12 \mathrm{~h}$ at $50{ }^{\circ} \mathrm{C}$ ) as a white solid.

M.p.: $108.4^{\circ} \mathrm{C}$

${ }^{1} \mathbf{H}-\mathrm{NMR}\left(\mathbf{6 0 0} \mathrm{MHz}, \mathbf{C D C l}_{3}\right.$, ppm) $\delta=8.14(\mathrm{~d}, J=8.2 \mathrm{~Hz}, 2 \mathrm{H}), 7.79(\mathrm{~d}, J=8.6 \mathrm{~Hz}, 2 \mathrm{H}), 7.74(\mathrm{~d}, J=$ $8.7 \mathrm{~Hz}, 2 \mathrm{H}), 7.46$ (d, $J=8.7 \mathrm{~Hz}, 2 \mathrm{H}), 4.41$ (q, $J=7.1 \mathrm{~Hz}, 2 \mathrm{H}), 1.41(\mathrm{t}, J=7.1 \mathrm{~Hz}, 3 \mathrm{H})$.

${ }^{13} \mathrm{C}-\mathrm{NMR}\left(\mathbf{1 5 0} \mathrm{MHz}, \mathrm{CDCl}_{3}, \mathbf{p p m}\right) \delta=194.8,165.7,140.8,139.5,135.2,133.8,131.4,129.5$ (2C), $128.8,61.5,14.3$.

IR $\left(\mathbf{A T R}, \mathbf{c m}^{-1}\right) \tilde{v}=1714,1648,1586,1568,1401,1368,1301,1268,1144,1105,1091,1010,978$, $931,875,853,841,783,737,708,668$.

MS (EI, 70 eV, \%) m/z = 290 (19), 289 (11), 288 (56), 260 (14), 245 (18), 243 (51), 180 (11), 177 (59), 152 (11), 149 (17), 14 (34), 139 (100), 111 (25), 76 (11), 75 (10), 71 (34), 70 (14), 57 (46), 56 (28), 43 (82), 42 (47), 41 (40). 
HRMS (EI, $70 \mathbf{~ e V}$ ) m/z: calc. for $\mathbf{C}_{16} \mathbf{H}_{13} \mathbf{C l O}_{3}$ : 288.0553; found 282.0549 .

Preparation of ethyl 4-(4-(tert-butyl)benzoyl)benzoate (5b)

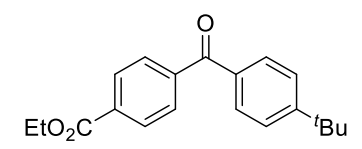

Based on TP4, $\mathrm{FeCl}_{2}$ (6.34 mg, $0.05 \mathrm{mmol}, 5.0 \mathrm{~mol} \%$ ), 4-tert-butylbenzoyl chloride (2b, $197 \mathrm{mg}$, $0.20 \mathrm{~mL}, 1.00 \mathrm{mmol}, 1.00$ equiv) and freshly distilled THF $(1.0 \mathrm{~mL})$ were used. The (4-(ethoxycarbonyl)phenyl)zinc(II) chloride (4a, $3.90 \mathrm{~mL}, 1.25 \mathrm{mmol}, 0.32 \mathrm{M}$ in THF, 1.25 equiv) was added dropwise and the reaction mixture was stirred for $4 \mathrm{~h}$ at $50{ }^{\circ} \mathrm{C}$. The reaction was quenched with a saturated aqueous $\mathrm{NH}_{4} \mathrm{Cl}$ solution and the aqueous layer was extracted with EtOAc $(3 \times 75 \mathrm{~mL})$. The combined organic layers were dried over $\mathrm{MgSO}_{4}$, filtered and concentrated under reduced pressure. The crude product was purified by flash column chromatography $\left(\mathrm{SiO}_{2}, i\right.$-hexane : EtOAc $=19: 1, \mathrm{R}_{\mathrm{f}}=$ 0.29 ) leading to the desired product $\mathbf{5 b}$ ( $203 \mathrm{mg}, 0.65 \mathrm{mmol}, 65 \%)$ as a pale yellow solid.

M.p.: $87.8^{\circ} \mathrm{C}$

${ }^{1} \mathrm{H}-\mathrm{NMR}\left(400 \mathrm{MHz}, \mathbf{C D C l}_{3}, \mathbf{p p m}\right) \delta=8.07(\mathrm{~d}, J=8.6 \mathrm{~Hz}, 2 \mathrm{H}), 7.76(\mathrm{~d}, J=8.6 \mathrm{~Hz}, 2 \mathrm{H}), 7.68(\mathrm{~d}, J=$ $8.7 \mathrm{~Hz}, 2 \mathrm{H}), 7.43(\mathrm{~d}, J=8.7 \mathrm{~Hz}, 2 \mathrm{H}), 4.35$ (q, $J=7.1 \mathrm{~Hz}, 2 \mathrm{H}), 1.35$ (t, $J=7.1 \mathrm{~Hz}, 3 \mathrm{H}), 1.29$ (s, 9H).

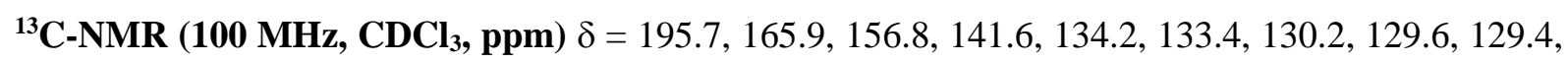
$125.4,61.4,35.2,31.1,14.3$.

IR $\left(\mathbf{A T R}, \mathbf{c m}^{-1}\right) \tilde{v}=2966,2906,2871,1712,1651,1605,1476,1462,1364,1278,1265,1191,1120$, 1106, 1015, 932, 874, 843, 791, 759, 745, 715, 702, 673.

MS (EI, 70 eV, \%) m/z = 311 (21), 310 (100), 297 (14), 296 (68), 266 (16), 265 (39), 178 (10), 177 (42), 145 (11), 125 (12), 121 (13), 118 (12), 115 (10), 111 (35), 104 (14), 76 (12), 65 (10).

HRMS (EI, $70 \mathbf{~ e V ) ~ m / z : ~ c a l c . ~ f o r ~} \mathbf{C}_{20} \mathbf{H}_{22} \mathbf{O}_{3}: 310.1569$; found 310.1558 .

\section{Preparation of ethyl 4-(4-fluorobenzoyl)benzoate (5c)}

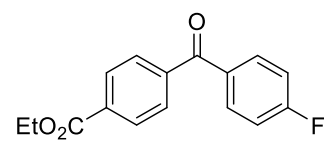

According to TP4, $\mathrm{FeCl}_{2}$ (6.34 mg, $0.05 \mathrm{mmol}, 5.0 \mathrm{~mol} \%$ ), 4-fluorobenzoyl chloride (2c, $159 \mathrm{mg}$, $0.12 \mathrm{~mL}, 1.00 \mathrm{mmol}, 1.00$ equiv) and freshly distilled THF (1.0 mL) were used. The (4-(ethoxy- 
carbonyl)phenyl)zinc(II) chloride (4a, $3.90 \mathrm{~mL}, 1.25 \mathrm{mmol}, 0.32 \mathrm{M}$ in THF, 1.25 equiv) was added dropwise and the reaction mixture was stirred for $4 \mathrm{~h}$ at $50{ }^{\circ} \mathrm{C}$. The reaction was quenched with a saturated aqueous $\mathrm{NH}_{4} \mathrm{Cl}$ solution and the aqueous layer was extracted with EtOAc $(3 \times 75 \mathrm{~mL})$. The combined organic layers were dried over $\mathrm{MgSO}_{4}$, filtered and concentrated under reduced pressure. Purification of the crude product by flash column chromatography $\left(\mathrm{SiO}_{2}, i\right.$-hexane $:$ EtOAc $=19: 1, \mathrm{R}_{\mathrm{f}}$ $=0.33)$ gave the product $\mathbf{5 c}(227 \mathrm{mg}, 0.83 \mathrm{mmol}, 83 \%)(21 \%$ isolated yield without catalyst after prolonged reaction time of $12 \mathrm{~h}$ at $50{ }^{\circ} \mathrm{C}$ ) as a white solid.

M.p.: $75.7^{\circ} \mathrm{C}$

${ }^{1} \mathbf{H}-N M R\left(600 ~ M H z, \mathbf{C D C l}_{3}, \mathbf{p p m}\right) \delta=8.14(\mathrm{~d}, J=8.6 \mathrm{~Hz}, 2 \mathrm{H}), 7.83(\mathrm{dd}, J=8.9,5.4 \mathrm{~Hz}, 2 \mathrm{H}), 7.79(\mathrm{~d}$, $J=8.6 \mathrm{~Hz}, 2 \mathrm{H}), 7.16(\mathrm{t}, J=8.6 \mathrm{~Hz}, 2 \mathrm{H}), 4.41(\mathrm{q}, J=7.1 \mathrm{~Hz}, 2 \mathrm{H}), 1.41(\mathrm{t}, J=7.1 \mathrm{~Hz}, 3 \mathrm{H})$.

${ }^{13}$ C-NMR (150 MHz, CDCl 3 , ppm) $\delta=194.5,165.6\left(\mathrm{~d},{ }^{1} J(\mathrm{C}, \mathrm{F})=254 \mathrm{~Hz}\right), 165.7,141.1,133.6,133.2$, $132.7\left(\mathrm{~d},{ }^{3} J(\mathrm{C}, \mathrm{F})=9.1 \mathrm{~Hz}\right), 130.1,129.5,127.2,115.7\left(\mathrm{~d},{ }^{2} J(\mathrm{C}, \mathrm{F})=22 \mathrm{~Hz}\right), 61.4,14.3$.

${ }^{19}$ F-NMR (376 MHz, $\left.\mathrm{CDCl}_{3}, \mathrm{ppm}\right) \delta=-105.0$.

IR $\left(\mathbf{A T R}, \mathbf{c m}^{-1}\right) \tilde{\mathrm{v}}=3069,2989,1712,1647,1596,1500,1407,1370,1308,1269,1232,1180,1148$, 1100, 1020, 1008, 978, 960, 934, 872, 856, 817, 787, 742, 718, 702, 672.

MS (EI, 70 eV, \%) m/z = 273 (2), 272 (11), 253 (11), 226 (12), 123 (25), 70 (13), 61 (17), 45 (15), 42 (100).

HRMS (EI, $70 \mathbf{~ e V ) ~ m / z : ~ c a l c . ~ f o r ~} \mathbf{C}_{\mathbf{1 6}} \mathbf{H}_{\mathbf{1 3}} \mathbf{F O}_{\mathbf{3}}$ : 272.0849; found 272.0837 .

\section{Preparation of ethyl 4-(4-bromobenzoyl)benzoate (5d)}

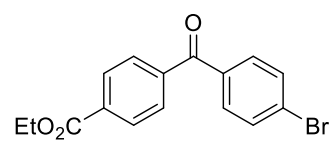

According to TP4, $\mathrm{FeCl}_{2}(6.34 \mathrm{mg}, 0.05 \mathrm{mmol}, 5.0 \mathrm{~mol} \%$ ), 4-bromobenzoyl chloride (2f, $220 \mathrm{mg}, 1.00$ mmol, 1.00 equiv) and freshly distilled THF $(1.0 \mathrm{~mL})$ were used. The (4-(ethoxycarbonyl)phenyl)zinc(II) chloride (4a, $3.90 \mathrm{~mL}, 1.25 \mathrm{mmol}, 0.32 \mathrm{M}$ in THF, 1.25 equiv) was added dropwise and the reaction mixture was stirred for $4 \mathrm{~h}$ at $50{ }^{\circ} \mathrm{C}$. The reaction was quenched with a saturated aqueous $\mathrm{NH}_{4} \mathrm{Cl}$ solution and the aqueous layer was extracted with EtOAc $(3 \times 75 \mathrm{~mL})$. The combined organic layers were dried over $\mathrm{MgSO}_{4}$, filtered and concentrated under reduced pressure. The 
crude product was purified by flash column chromatography $\left(\mathrm{SiO}_{2}, i\right.$-hexane $:$ EtOAc $=19: 1, \mathrm{R}_{\mathrm{f}}=$ 0.22 ) affording the product $\mathbf{5 d}(227 \mathrm{mg}, 0.68 \mathrm{mmol}, 68 \%)$ as a white solid.

M.p.: $108.2^{\circ} \mathrm{C}$

${ }^{1} \mathrm{H}-\mathrm{NMR}\left(400 \mathrm{MHz}, \mathbf{C D C l}_{3}\right.$, ppm) $\delta=8.15(\mathrm{~d}, J=8.5 \mathrm{~Hz}, 2 \mathrm{H}), 7.80(\mathrm{~d}, J=8.5 \mathrm{~Hz}, 2 \mathrm{H}), 7.73-7.58(\mathrm{~m}$, $4 \mathrm{H}), 4.42(\mathrm{q}, J=7.1 \mathrm{~Hz}, 2 \mathrm{H}), 1.42(\mathrm{t}, J=7.1 \mathrm{~Hz}, 3 \mathrm{H})$.

${ }^{13}$ C-NMR (100 MHz, $\mathbf{C D C l}_{\mathbf{3}}$, ppm) $\delta=194.9,165.7,140.7,135.7,133.8,131.8,131.5,129.6,129.5$, 128.1, 61.4, 14.3.

IR $\left(A T R, \mathbf{c m}^{-1}\right) \tilde{v}=2984,2958,2932,1712,1648,1582,1478,1460,1396,1368,1266,1182,1142$, $1102,1068,1022,978,928,838,782,732,704,664$.

MS (EI, 70 eV, \%) m/z = 335 (12), 334 (54), 333 (12), 332 (57), 289 (10), 287 (44), 185 (99), 183 (100), 178 (12), 177 (98), 157 (20), 149 (26), 76 (50), 75 (24).

HRMS (EI, $70 \mathbf{~ e V}$ ) m/z: calc. for $\mathbf{C}_{\mathbf{1 6}} \mathbf{H}_{\mathbf{1 3}} \mathrm{BrO}_{\mathbf{3}}: 332.0048$; found 332.0043 .

Preparation of ethyl 3-(4-chlorobenzoyl)benzoate (5e)

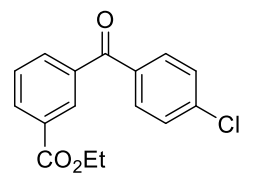

According to TP4, $\mathrm{FeCl}_{2}(6.34 \mathrm{mg}, 0.05 \mathrm{mmol}, 5.0 \mathrm{~mol} \%$ ), 4-chlorobenzoyl chloride (2a, $175 \mathrm{mg}$, $0.13 \mathrm{~mL}, 1.00 \mathrm{mmol}, 1.00$ equiv) and freshly distilled THF $(1.0 \mathrm{~mL})$ were used. The (3-(ethoxycarbonyl)phenyl)zinc(II) chloride $(\mathbf{4 b}, 4.00 \mathrm{~mL}, 1.25 \mathrm{mmol}, 0.31 \mathrm{M}$ in THF, 1.25 equiv) was added dropwise and the reaction mixture was stirred for $4 \mathrm{~h}$ at $50{ }^{\circ} \mathrm{C}$. The reaction was quenched with a saturated aqueous $\mathrm{NH}_{4} \mathrm{Cl}$ solution and the aqueous layer was extracted with EtOAc $(3 \times 75 \mathrm{~mL})$. The combined organic layers were dried over $\mathrm{MgSO}_{4}$, filtered and concentrated under reduced pressure. Purification of the crude product by flash column chromatography $\left(\mathrm{SiO}_{2}, i\right.$-hexane $:$ EtOAc $=9: 1, \mathrm{R}_{\mathrm{f}}$ $=0.36)$ furnished product $5 \mathbf{e}(217 \mathrm{mg}, 0.75 \mathrm{mmol}, 75 \%)$ as a pale yellow oil.

${ }^{1}$ H-NMR (400 MHz, CDCl 3 , ppm) $\delta=8.33(\mathrm{t}, J=1.7 \mathrm{~Hz}, 1 \mathrm{H}), 8.19(\mathrm{dt}, J=7.8,1.5 \mathrm{~Hz}, 1 \mathrm{H}), 7.88(\mathrm{dt}$, $J=7.7,1.7 \mathrm{~Hz}, 1 \mathrm{H}), 7.68(\mathrm{~d}, J=8.7 \mathrm{~Hz}, 2 \mathrm{H}), 7.40(\mathrm{~d}, J=8.7 \mathrm{~Hz}, 2 \mathrm{H}), 4.33(\mathrm{q}, J=7.1 \mathrm{~Hz}, 2 \mathrm{H}), 1.32(\mathrm{t}$, $J=7.1 \mathrm{~Hz}, 3 \mathrm{H})$.

${ }^{13}$ C-NMR (100 MHz, CDCl $\left.\mathbf{3}, \mathbf{p p m}\right) \delta=194.5,165.7,139.3,137.6,135.6,133.8,133.6,131.4,130.9$, $130.8,128.8,128.7,61.4,14.3$. 
IR $\left(\mathbf{A T R}, \mathbf{c m}^{-1}\right) \tilde{\mathrm{v}}=2982,1716,1660,1601,1584,1486,1399,1367,1302,1287,1239,1170,1150$, $1088,1014,974,960,874,844,775,734,700$.

MS (EI, 70 eV, \%) m/z = 290 (16), 289 (10), 288 (40), 245 (13), 243 (36), 177 (40), 148 (12), 141 (33), 139 (100), 111 (259, 76 (12), 75 (10), 44 (10).

HRMS (EI, $70 \mathrm{eV}$ ) m/z: calc. for $\mathbf{C}_{16} \mathbf{H}_{13} \mathbf{C l O}_{3}$ : 288.0553; found 288.0547 .

\section{Preparation of ethyl 3-(4-(tert-butyl)benzoyl)benzoate (5f)}

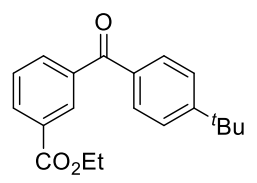

Based on TP4, $\mathrm{FeCl}_{2}$ (6.34 mg, $0.05 \mathrm{mmol}, 5.0 \mathrm{~mol} \%$ ), 4-tert-butylbenzoyl chloride (2b, $197 \mathrm{mg}$, $0.20 \mathrm{~mL}, 1.00 \mathrm{mmol}, 1.00$ equiv) and freshly distilled THF $(1.0 \mathrm{~mL})$ were used. The (3-(ethoxycarbonyl)phenyl)zinc(II) chloride (4b, $4.00 \mathrm{~mL}, 1.25 \mathrm{mmol}, 0.31 \mathrm{M}$ in THF, 1.25 equiv) was added dropwise and the reaction mixture was stirred for $4 \mathrm{~h}$ at $50{ }^{\circ} \mathrm{C}$. The reaction was quenched with a saturated aqueous $\mathrm{NH}_{4} \mathrm{Cl}$ solution and the aqueous layer was extracted with EtOAc $(3 \times 75 \mathrm{~mL})$. The combined organic layers were dried over $\mathrm{MgSO}_{4}$, filtered and concentrated under reduced pressure. Purification of the crude product by flash column chromatography $\left(\mathrm{SiO}_{2}, i\right.$-hexane $:$ EtOAc $=19: 1, \mathrm{R}_{\mathrm{f}}$ $=0.16)$ gave the product $\mathbf{5 f}(224 \mathrm{mg}, 0.72 \mathrm{mmol}, 72 \%)$ as a pale yellow oil.

${ }^{1} \mathrm{H}-\mathrm{NMR}\left(400 \mathrm{MHz}, \mathbf{C D C l}_{3}\right.$, ppm) $\delta=8.38(\mathrm{t}, J=1.5 \mathrm{~Hz}, 1 \mathrm{H}), 8.17(\mathrm{dt}, J=7.8,1.4 \mathrm{~Hz}, 1 \mathrm{H}), 7.90$ (dt, $J=7.7,1.5 \mathrm{~Hz}, 1 \mathrm{H}), 7.68(\mathrm{~d}, J=8.6 \mathrm{~Hz}, 2 \mathrm{H}), 7.49(\mathrm{~d}, J=7.7 \mathrm{~Hz}, 1 \mathrm{H}), 7.43(\mathrm{~d}, J=8.6 \mathrm{~Hz}, 2 \mathrm{H}), 4.32$ (q, $J=7.1 \mathrm{~Hz}, 2 \mathrm{H}), 1.32(\mathrm{t}, J=7.1 \mathrm{~Hz}, 3 \mathrm{H}), 1.29(\mathrm{~s}, 9 \mathrm{H})$.

${ }^{13}$ C-NMR (100 MHz, CDCl $\mathbf{3}$, ppm) $\delta=195.5,165.9,156.6,138.3,134.3,133.9,132.9,130.8,130.7$, 130.1, 128.4, 125.4, 61.3, 35.1, 31.1, 14.3.

IR $\left(A T R, \mathbf{c m}^{-1}\right) \tilde{v}=2965,2255,1719,1659,1605,1464,1407,1367,1317,1304,1286,1246,1193$, 1154, 1102, 1081, 1022, 975, 909, 876, 850, 781, 746, 730, 707, 675.

MS (EI, 70 eV, \%) m/z = 312 (14), 311 (69), 310 (30), 298 (13), 296 (19), 295 (100), 267 (35), 265 (14), 253 (19), 176 (14), 161 (70), 148 (30), 146 (10), 145 (11), 118 (12), 111 (13).

HRMS (EI, $70 \mathbf{~ e V}$ ) m/z: calc. for $\mathbf{C}_{20} \mathbf{H}_{22} \mathbf{O}_{3}: 310.1569$; found 310.1563 . 


\section{Preparation of ethyl 4-(cyclohexanecarbonyl)benzoate (5g)}

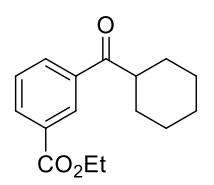

Based on TP4, $\mathrm{FeCl}_{2}(6.34 \mathrm{mg}, 0.05 \mathrm{mmol}, 5.0 \mathrm{~mol} \%)$, cyclohexanecarbonyl chloride (2h, $145 \mathrm{mg}$, $0.13 \mathrm{~mL}, 1.00 \mathrm{mmol}, 1.00$ equiv) and freshly distilled THF (1.0 mL) were used. The (3-(ethoxycarbonyl)phenyl)zinc(II) chloride (4b, $4.00 \mathrm{~mL}, 1.25 \mathrm{mmol}, 0.31 \mathrm{M}$ in THF, 1.25 equiv) was added dropwise and the reaction mixture was stirred for $4 \mathrm{~h}$ at $50{ }^{\circ} \mathrm{C}$. The reaction was quenched with a saturated aqueous $\mathrm{NH}_{4} \mathrm{Cl}$ solution and the aqueous layer was extracted with EtOAc $(3 \times 75 \mathrm{~mL})$. The combined organic layers were dried over $\mathrm{MgSO}_{4}$, filtered and concentrated under reduced pressure. The crude product was purified by flash column chromatography $\left(\mathrm{SiO}_{2}, i\right.$-hexane $:$ EtOAc $\left.=9: 1, \mathrm{R}_{\mathrm{f}}=0.50\right)$ leading to the product $\mathbf{5 g}(155 \mathrm{mg}, 0.60 \mathrm{mmol}, 60 \%)$ as a colourless oil.

${ }^{1} \mathrm{H}-\mathrm{NMR}\left(\mathbf{8 0 0} \mathbf{M H z}, \mathbf{C D C l}_{3}, \mathbf{p p m}\right) \delta=8.59(\mathrm{t}, J=1.6 \mathrm{~Hz}, 1 \mathrm{H}), 8.24(\mathrm{dt}, J=7.7,1.4 \mathrm{~Hz}, 1 \mathrm{H}), 8.14(\mathrm{dt}$, $J=7.8,1.5 \mathrm{~Hz}, 1 \mathrm{H}), 7.57(\mathrm{t}, J=7.7 \mathrm{~Hz}, 1 \mathrm{H}), 4.44(\mathrm{q}, J=7.1 \mathrm{~Hz}, 2 \mathrm{H}), 3.32(\mathrm{tt}, J=11.4,3.3 \mathrm{~Hz}, 1 \mathrm{H})$, 1.93-1.89 (m, 2H), $1.87(\mathrm{dt}, J=13.4,3.5 \mathrm{~Hz}, 2 \mathrm{H}), 1.79-1.73(\mathrm{~m}, 1 \mathrm{H}), 1.52(\mathrm{qd}, J=13.0,3.2 \mathrm{~Hz}, 2 \mathrm{H})$, $1.44(\mathrm{t}, J=7.1 \mathrm{~Hz}, 3 \mathrm{H}), 1.30(\mathrm{dtd}, J=12.9,9.1,4.6 \mathrm{~Hz}, 2 \mathrm{H})$.

${ }^{13}$ C-NMR (200 MHz, $\mathbf{C D C l}_{3}$, ppm) $\delta=203.3,166.1,136.8,133.6,132.5,131.2,129.4,128.9,61.5$, $45.8,29.5,26.1,25.9,14.5$.

IR $\left(A T R, \mathbf{c m}^{-1}\right) \tilde{v}=2929,2853,1719,1681,1601,1447,1367,1295,1276,1238,1198,1172,1135$, 1100, 1077, 1022, 990, 981, 892, 864, 821, 760, 714, 693.

MS (EI, 70 eV, \%) m/z = 260 (1), 215 (11), 187 (24), 178 (12), 177 (100), 149 (14), 83 (11), 55 (13).

HRMS (EI, $70 \mathbf{~ e V ) ~ m / z : ~ c a l c . ~ f o r ~} \mathbf{C}_{16} \mathbf{H}_{20} \mathbf{O}_{3}$ : 260.1412; found 260.1407 .

\section{Preparation of ethyl 2-(4-chlorobenzoyl)benzoate (5h)}

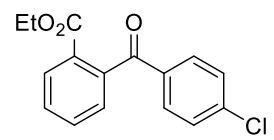

According to $\mathbf{T P 4}, \mathrm{FeCl}_{2}(6.34 \mathrm{mg}, 0.05 \mathrm{mmol}, 5.0 \mathrm{~mol} \%)$, 4-chlorobenzoyl chloride (2a, $175 \mathrm{mg}$, $0.13 \mathrm{~mL}, 1.00 \mathrm{mmol}, 1.00$ equiv) and freshly distilled THF $(1.0 \mathrm{~mL})$ were used. The (2-(ethoxycarbonyl)phenyl)zinc(II) chloride (4c, $3.70 \mathrm{~mL}, 1.25 \mathrm{mmol}, 0.34 \mathrm{M}$ in THF, 1.25 equiv) was added dropwise and the reaction mixture was stirred for $4 \mathrm{~h}$ at $50{ }^{\circ} \mathrm{C}$. The reaction was quenched with a saturated aqueous $\mathrm{NH}_{4} \mathrm{Cl}$ solution and the aqueous layer was extracted with EtOAc $(3 \times 75 \mathrm{~mL})$. The 
combined organic layers were dried over $\mathrm{MgSO}_{4}$, filtered and concentrated under reduced pressure. Purification of the crude product by flash column chromatography $\left(\mathrm{SiO}_{2}, i\right.$-hexane $:$ EtOAc $=19: 1, \mathrm{R}_{\mathrm{f}}$ $=0.18)$ afforded product $\mathbf{5 h}(213 \mathrm{mg}, 0.74 \mathrm{mmol}, 74 \%)$ as a white solid.

M.p.: $78.2^{\circ} \mathrm{C}$

${ }^{1} \mathrm{H}-\mathrm{NMR}\left(400 \mathrm{MHz}, \mathbf{C D C l}_{3}\right.$, ppm) $\delta=8.07(\mathrm{dd}, J=7.8,1.9 \mathrm{~Hz}, 1 \mathrm{H}), 7.69(\mathrm{~d}, J=8.8 \mathrm{~Hz}, 2 \mathrm{H}), 7.67-$ $7.52(\mathrm{~m}, 2 \mathrm{H}), 7.42-7.34(\mathrm{~m}, 3 \mathrm{H}), 4.11(\mathrm{q}, J=7.1 \mathrm{~Hz}, 2 \mathrm{H}), 1.10(\mathrm{t}, J=7.1 \mathrm{~Hz}, 3 \mathrm{H})$.

${ }^{13}$ C-NMR (100 MHz, CDCl $\mathbf{3}$, ppm) $\delta=195.9,165.8,141.3,139.6,135.8,132.6,130.8,130.4,129.8$, $129.4,128.9,127.6,61.7,13.9$.

IR $\left(A T R, \mathbf{c m}^{-1}\right) \tilde{v}=3070,2988,2938,1710,1676,1584,1576,1486,1474,1446,1398,1364,1282$, 1260, 1166, 1140, 1086, 1016, 962, 928, 876, 848, 828, 768, 750, 708, 686.

MS (EI, 70 eV, \%) m/z = 290 (11), 288 (37), 245 (17), 244 (15), 177 (44), 154 (33), 149 (100), 139 (76), 111 (27).

HRMS (EI, $70 \mathbf{~ e V}$ ) m/z: calc. for $\mathbf{C}_{16} \mathbf{H}_{13} \mathbf{C l O}_{3}$ : 288.0553; found 288.0551.

\section{Preparation of ethyl 2-(4-cyanobenzoyl)benzoate (5i)}

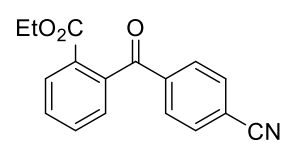

According to TP4, $\mathrm{FeCl}_{2}(6.34 \mathrm{mg}, 0.05 \mathrm{mmol}, 5.0 \mathrm{~mol} \%$ ), 4-cyanobenzoyl chloride (2g, $166 \mathrm{mg}, 1.00$ mmol, 1.00 equiv) and freshly distilled THF $(1.0 \mathrm{~mL})$ were used. The (2-(ethoxycarbonyl)phenyl)zinc(II) chloride (4c, $3.70 \mathrm{~mL}, 1.25 \mathrm{mmol}, 0.34 \mathrm{M}$ in THF, 1.25 equiv) was added dropwise and the reaction mixture was stirred for $4 \mathrm{~h}$ at $50{ }^{\circ} \mathrm{C}$. The reaction was quenched with a saturated aqueous $\mathrm{NH}_{4} \mathrm{Cl}$ solution and the aqueous layer was extracted with EtOAc $(3 \times 75 \mathrm{~mL})$. The combined organic layers were dried over $\mathrm{MgSO}_{4}$, filtered and concentrated under reduced pressure. The crude product was purified by flash column chromatography $\left(\mathrm{SiO}_{2}, i\right.$-hexane $:$ EtOAc $\left.=9: 1, \mathrm{R}_{\mathrm{f}}=0.13\right)$ yielding the product $\mathbf{5 i}(173 \mathrm{mg}, 0.62 \mathrm{mmol}, 62 \%)$ as a pale yellow solid.

M.p.: $148.6^{\circ} \mathrm{C}$

${ }^{1} \mathbf{H}-\mathrm{NMR}\left(\mathbf{4 0 0} \mathbf{M H z}, \mathbf{C D C l}_{3}, \mathbf{p p m}\right) \delta=8.09(\mathrm{dd}, J=7.8,1.3 \mathrm{~Hz}, 1 \mathrm{H}), 7.83(\mathrm{~d}, J=8.6 \mathrm{~Hz}, 2 \mathrm{H}), 7.72(\mathrm{~d}$, $J=8.6 \mathrm{~Hz}, 2 \mathrm{H}), 7.67(\mathrm{td}, J=7.5,1.4 \mathrm{~Hz}, 1 \mathrm{H}), 7.61(\mathrm{td}, J=7.6,1.4 \mathrm{~Hz}, 1 \mathrm{H}), 4.11(\mathrm{q}, J=7.1 \mathrm{~Hz}, 2 \mathrm{H})$, $1.12(\mathrm{t}, J=7.1 \mathrm{~Hz}, 3 \mathrm{H})$. 
${ }^{13}$ C-NMR (100 MHz, CDCl 3 , ppm) $\delta=195.6,165.6,140.8,140.5,132.9,132.5,130.4,130.2,129.6$, 129.4, 127.6, 118.1, 116.3, 61.8, 13.9.

IR $\left(\mathbf{A T R}, \mathbf{c m}^{-1}\right) \tilde{\mathrm{v}}=3096,2992,2230,1956,1708,1680,1602,1574,1476,1408,1368,1276,1262$, $1144,1114,1084,1018,932,854,760,710,690$.

MS (EI, 70 eV, \%) m/z = 279 (13), 235 (17), 234 (59), 177 (62), 149 (100), 130 (23), 102 (22).

HRMS (EI, $70 \mathbf{~ e V ) ~ m / z : ~ c a l c . ~ f o r ~} \mathbf{C}_{\mathbf{1 7}} \mathbf{H}_{\mathbf{1 3}} \mathbf{N O}_{\mathbf{3}}$ : 279.0895; found 279.0890 .

\section{Preparation of (4-(tert-butyl)phenyl)(4-(trifluoromethyl)phenyl)methanone (5j)}

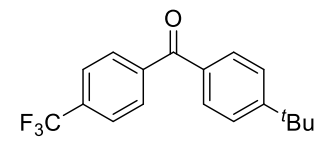

Based on TP4, $\mathrm{FeCl}_{2}$ (6.34 mg, $0.05 \mathrm{mmol}, 5.0 \mathrm{~mol} \%$ ), 4-tert-butylbenzoyl chloride (2b, $197 \mathrm{mg}$, $0.20 \mathrm{~mL}, 1.00 \mathrm{mmol}, 1.00$ equiv) and freshly distilled THF $(1.0 \mathrm{~mL})$ were used. The (4-(trifluoromethyl)phenyl)zinc(II) chloride (4d, $3.00 \mathrm{~mL}, 1.25 \mathrm{mmol}, 0.42 \mathrm{M}$ in THF, 1.25 equiv) was added dropwise and the reaction mixture was stirred for $3 \mathrm{~h}$ at $50{ }^{\circ} \mathrm{C}$. The reaction was quenched with a saturated aqueous $\mathrm{NH}_{4} \mathrm{Cl}$ solution and the aqueous layer was extracted with EtOAc $(3 \times 75 \mathrm{~mL})$. The combined organic layers were dried over $\mathrm{MgSO}_{4}$, filtered and concentrated under reduced pressure. Purification of the crude product by flash column chromatography $\left(\mathrm{SiO}_{2}, i\right.$-hexane $:$ EtOAc $=99: 1, \mathrm{R}_{\mathrm{f}}$ $=0.50)$ gave the product $5 \mathbf{j}(255 \mathrm{mg}, 0.83 \mathrm{mmol}, 83 \%)$ as a white solid.

M.p.: $76.0^{\circ} \mathrm{C}$

${ }^{1} \mathrm{H}-\mathrm{NMR}\left(400 \mathrm{MHz}, \mathbf{C D C l}_{3}, \mathbf{p p m}\right) \delta=7.82(\mathrm{~d}, J=8.0 \mathrm{~Hz}, 2 \mathrm{H}), 7.74-7.64(\mathrm{~m}, 4 \mathrm{H}), 7.45(\mathrm{~d}, J=8.0 \mathrm{~Hz}$, 2H), 1.31 (s, 9H).

${ }^{13}$ C-NMR (100 MHz, $\left.\mathbf{C D C l}_{3}, \mathbf{p p m}\right) \delta=195.2,157.0,141.0\left(\mathrm{q},{ }^{4} J(\mathrm{C}, \mathrm{F})=2.0 \mathrm{~Hz}\right), 134.0,133.5$ (q, $\left.{ }^{2} J(\mathrm{C}, \mathrm{F})=32 \mathrm{~Hz}\right), 130.2,130.0,125.5,125.3\left(\mathrm{q},{ }^{3} J(\mathrm{C}, \mathrm{F})=3.8 \mathrm{~Hz}\right), 123.7\left(\mathrm{q},{ }^{1} J(\mathrm{C}, \mathrm{F})=271 \mathrm{~Hz}\right), 35.2$, 31.1 .

${ }^{19}$ F-NMR (376 MHz, $\left.\mathrm{CDCl}_{3}, \mathbf{p p m}\right) \delta=-63.0$.

IR $\left(\mathbf{A T R}, \mathbf{c m}^{-1}\right) \tilde{\mathrm{v}}=2969,1651,1604,1408,1324,1310,1280,1163,1122,1105,1064,1016,972$, 932, 860, 838, 774, 734, 698, 677. 
MS (EI, 70 eV, \%) m/z = 306 (2), 291 (15), 162 (13), 161 (100).

HRMS (EI, $70 \mathbf{~ e V}$ ) m/z: calc. for $\mathbf{C}_{18} \mathbf{H}_{17} \mathbf{F}_{3} \mathbf{O}$ : 306.1231; found 306.1233 .

Preparation of (4-methoxyphenyl)(4-(trifluoromethyl)phenyl)methanone (5k)

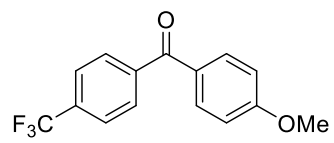

Based on TP4, $\mathrm{FeCl}_{2}(6.34 \mathrm{mg}, 0.05 \mathrm{mmol}, 5.0 \mathrm{~mol} \%)$, 4-methoxybenzoyl chloride (2e, $171 \mathrm{mg}$, $1.00 \mathrm{mmol}, 1.00$ equiv) and freshly distilled THF $(1.0 \mathrm{~mL})$ were used. The (4-(trifluoromethyl)phenyl)zinc(II) chloride (4d, $3.00 \mathrm{~mL}, 1.25 \mathrm{mmol}, 0.42 \mathrm{M}$ in THF, 1.25 equiv) was added dropwise and the reaction mixture was stirred for $3 \mathrm{~h}$ at $50{ }^{\circ} \mathrm{C}$. The reaction was quenched with a saturated aqueous $\mathrm{NH}_{4} \mathrm{Cl}$ solution and the aqueous layer was extracted with EtOAc $(3 \times 75 \mathrm{~mL})$. The combined organic layers were dried over $\mathrm{MgSO}_{4}$, filtered and concentrated under reduced pressure. The crude product was purified by flash column chromatography $\left(\mathrm{SiO}_{2}, i\right.$-hexane : EtOAc $=19: 1, \mathrm{R}_{\mathrm{f}}=$ $0.25)$ leading to the product $\mathbf{5 k}(173 \mathrm{mg}, 0.62 \mathrm{mmol}, 62 \%)$ as a white solid.

M.p.: $124.1^{\circ} \mathrm{C}$

${ }^{1} \mathrm{H}-\mathrm{NMR}\left(400 \mathrm{MHz}, \mathbf{C D C l}_{3}, \mathbf{p p m}\right) \delta=7.78-7.74(\mathrm{~m}, 4 \mathrm{H}), 7.67(\mathrm{~d}, J=8.2 \mathrm{~Hz}, 2 \mathrm{H}), 6.91(\mathrm{~d}, J=8.9 \mathrm{~Hz}$, $2 \mathrm{H}), 3.83(\mathrm{~s}, 3 \mathrm{H})$.

${ }^{13} \mathrm{C}-\mathrm{NMR}\left(100 \mathrm{MHz}, \mathrm{CDCl}_{3}\right.$, ppm) $\delta=194.3,163.7,141.5\left(\mathrm{q},{ }^{4} J(\mathrm{C}, \mathrm{F})=2.0 \mathrm{~Hz}\right), 133.3\left(\mathrm{q},{ }^{2} J(\mathrm{C}, \mathrm{F})=\right.$ $33 \mathrm{~Hz}), 132.6,129.8,129.4,125.3\left(\mathrm{q},{ }^{3} J(\mathrm{C}, \mathrm{F})=4.0 \mathrm{~Hz}\right), 123.7\left(\mathrm{q},{ }^{1} J(\mathrm{C}, \mathrm{F})=271 \mathrm{~Hz}\right), 113.8,55.6$.

${ }^{19}$ F-NMR (376 MHz, $\left.\mathrm{CDCl}_{3}, \mathrm{ppm}\right) \delta=-63.0$.

IR $\left(\mathbf{A T R}, \mathbf{c m}^{-1}\right) \tilde{v}=1643,1600,1573,1504,1407,1328,1306,1286,1262,1165,1127,1108,1067$, 1029, 1016, 972, 930, 861, 843, 770, 736, 700, 686.

MS (EI, 70 eV, \%) m/z = 280 (26), 145 (14), 135 (100), 92 (13), 77 (16)

HRMS (EI, $70 \mathbf{~ e V ) ~ m / z : ~ c a l c . ~ f o r ~} \mathbf{C}_{15} \mathbf{H}_{11} \mathbf{F}_{3} \mathbf{O}_{2}$ : 280.0711; found 280.0707. 
Preparation of (4-chlorophenyl)(4-fluoro-3-methylphenyl)methanone (5l)

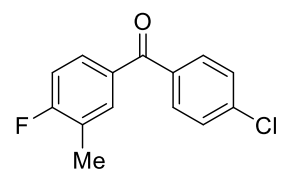

According to $\mathbf{T P 4}, \mathrm{FeCl}_{2}(6.34 \mathrm{mg}, 0.05 \mathrm{mmol}, 5.0 \mathrm{~mol} \%)$, 4-chlorobenzoyl chloride (2a, $175 \mathrm{mg}$, $0.13 \mathrm{~mL}, 1.00 \mathrm{mmol}, 1.00$ equiv) and freshly distilled THF (1.0 mL) were used. The (4-fluoro-3methylphenyl)zinc(II) chloride $(4 \mathrm{e}, 3.80 \mathrm{~mL}, 1.25 \mathrm{mmol}, 0.33 \mathrm{M}$ in THF, 1.25 equiv) was added dropwise and the reaction mixture was stirred for $2 \mathrm{~h}$ at $50{ }^{\circ} \mathrm{C}$. The reaction was quenched with a saturated aqueous $\mathrm{NH}_{4} \mathrm{Cl}$ solution and the aqueous layer was extracted with EtOAc $(3 \times 75 \mathrm{~mL})$. The combined organic layers were dried over $\mathrm{MgSO}_{4}$, filtered and concentrated under reduced pressure. Purification of the crude product by flash column chromatography $\left(\mathrm{SiO}_{2}, i\right.$-hexane $:$ EtOAc $=99: 1, \mathrm{R}_{\mathrm{f}}$ $=0.40)$ afforded product $\mathbf{5 l}(188 \mathrm{mg}, 0.76 \mathrm{mmol}, 76 \%)$ as a white solid.

M.p.: $124.8^{\circ} \mathrm{C}$

${ }^{1} \mathrm{H}-\mathrm{NMR}\left(\mathbf{6 0 0} \mathrm{MHz}, \mathbf{C D C l}_{3}, \mathbf{p p m}\right) \delta=7.70(\mathrm{~d}, J=8.6 \mathrm{~Hz}, 2 \mathrm{H}), 7.66(\mathrm{dd}, J=7.8,1.6 \mathrm{~Hz}, 1 \mathrm{H}), 7.58$ (ddd, $J=7.4,4.8,2.1 \mathrm{~Hz}, 1 \mathrm{H}), 7.45(\mathrm{~d}, J=8.7 \mathrm{~Hz}, 2 \mathrm{H}), 7.08(\mathrm{t}, J=8.8 \mathrm{~Hz}, 1 \mathrm{H}), 2.32(\mathrm{~s}, 3 \mathrm{H})$.

${ }^{13}$ C-NMR (150 MHz, CDCl 3 , ppm) $\delta=194.3,164.1\left(\mathrm{~d},{ }^{1} J(\mathrm{C}, \mathrm{F})=253 \mathrm{~Hz}\right), 138.8,135.9,133.6(\mathrm{~d}$, $\left.{ }^{3} J(\mathrm{C}, \mathrm{F})=6.0 \mathrm{~Hz}\right), 133.2,131.2,129.9\left(\mathrm{~d},{ }^{3} J(\mathrm{C}, \mathrm{F})=9.0 \mathrm{~Hz}\right), 128.6,125.4\left(\mathrm{~d},{ }^{2} J(\mathrm{C}, \mathrm{F})=18 \mathrm{~Hz}\right), 115.0(\mathrm{~d}$, $\left.{ }^{2} J(\mathrm{C}, \mathrm{F})=23 \mathrm{~Hz}\right), 14.5$.

${ }^{19}$ F-NMR (376 MHz, $\left.\mathrm{CDCl}_{3}, \mathbf{p p m}\right) \delta=-109.6$.

IR $\left(A T R\right.$, cm$\left.^{-1}\right) \tilde{v}=2959,2927,2870,1724,1647,1605,1582,1486,1398,1300,1281,1262,1238$, 1190, 1176, 1134, 1113, 1086, 1007, 972, 915, 858, 842, 828, 759, 751, 684.

MS (EI, 70 eV, \%) m/z = 250 (24), 249 (12), 248 (76), 213 (13), 141 (13), 139 (42), 137 (100), 111 (14), 109 (18).

HRMS (EI, $70 \mathbf{~ e V ) ~ m / z : ~ c a l c . ~ f o r ~} \mathbf{C}_{\mathbf{1 4}} \mathbf{H}_{\mathbf{1 0}}$ ClFO: 248.0404; found 248.0396.

Preparation of (4-(tert-butyl)phenyl)(4-fluoro-3-methylphenyl)methanone (5m)

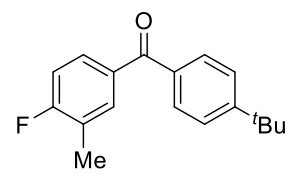


According to TP4, $\mathrm{FeCl}_{2}$ (6.34 mg, $0.05 \mathrm{mmol}, 5.0 \mathrm{~mol} \%$ ), 4-tert-butylbenzoyl chloride (2b, $197 \mathrm{mg}$, $0.20 \mathrm{~mL}, 1.00 \mathrm{mmol}, 1.00$ equiv) and freshly distilled THF (1.0 mL) were used. The (4-fluoro-3methylphenyl)zinc(II) chloride $(4 \mathrm{e}, 3.80 \mathrm{~mL}, 1.25 \mathrm{mmol}, 0.33 \mathrm{M}$ in THF, 1.25 equiv) was added dropwise and the reaction mixture was stirred for $2 \mathrm{~h}$ at $50{ }^{\circ} \mathrm{C}$. The reaction was quenched with a saturated aqueous $\mathrm{NH}_{4} \mathrm{Cl}$ solution and the aqueous layer was extracted with EtOAc $(3 \times 75 \mathrm{~mL})$. The combined organic layers were dried over $\mathrm{MgSO}_{4}$, filtered and concentrated under reduced pressure. Purification of the crude product by flash column chromatography $\left(\mathrm{SiO}_{2}, i\right.$-hexane $:$ EtOAc $=19: 1, \mathrm{R}_{\mathrm{f}}$ $=0.34)$ furnished product $5 \mathrm{~m}(189 \mathrm{mg}, 0.70 \mathrm{mmol}, 70 \%)$ as a colourless oil.

${ }^{1} \mathrm{H}-\mathrm{NMR}\left(600 \mathrm{MHz}, \mathbf{C D C l}_{3}, \mathbf{p p m}\right) \delta=7.73-7.70(\mathrm{~m}, 2 \mathrm{H}), 7.65-7.59(\mathrm{~m}, 1 \mathrm{H}), 7.50(\mathrm{~d}, J=8.4 \mathrm{~Hz}, 2 \mathrm{H})$, $7.08(\mathrm{~d}, J=8.9 \mathrm{~Hz}, 1 \mathrm{H}), 2.34(\mathrm{~s}, 3 \mathrm{H}), 1.37$ (s, 9H).

${ }^{13}$ C-NMR (150 MHz, CDCl 3 , ppm) $\delta=195.4,164.0\left(\mathrm{~d},{ }^{1} J(\mathrm{C}, \mathrm{F})=252 \mathrm{~Hz}\right), 156.3,135.0,134.0,133.8$ $\left(\mathrm{d},{ }^{3} J(\mathrm{C}, \mathrm{F})=5.4 \mathrm{~Hz}\right), 130.1,125.4,125.1,115.0\left(\mathrm{~d},{ }^{2} J(\mathrm{C}, \mathrm{F})=23 \mathrm{~Hz}\right), 35.2,31.3,14.7$.

${ }^{19}$ F-NMR (376 MHz, $\left.\mathrm{CDCl}_{3}, \mathrm{ppm}\right) \delta=-110.7$.

IR $\left(\mathbf{A T R}, \mathbf{c m}^{-1}\right) \tilde{v}=2963,2870,1718,1656,1605,1498,1407,1364,1315,1300,1282,1264,1239$, 1185, 1115, 1018, 968, 905, 850, 827, 772, 757, 726, 692.

MS (EI, 70 eV, \%) m/z = 271 (7), 270 (35), 256 (25), 255 (100), 137 (11).

HRMS (EI, 70 eV) m/z: calc. for $\mathbf{C}_{18} \mathbf{H}_{19} \mathbf{F O}$ : 270.1420; found 270.1419 .

\section{Preparation of (4-fluoro-3-methylphenyl)(4-methoxyphenyl)methanone (5n)}

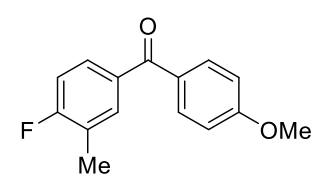

According to TP4, $\mathrm{FeCl}_{2}(6.34 \mathrm{mg}, 0.05 \mathrm{mmol}, 5.0 \mathrm{~mol} \%$ ), 4-methoxybenzoyl chloride (2e, $171 \mathrm{mg}$, $1.00 \mathrm{mmol}, 1.00$ equiv) and freshly distilled THF $(1.0 \mathrm{~mL})$ were used. The (4-fluoro-3methylphenyl)zinc(II) chloride (4e, $3.80 \mathrm{~mL}, 1.25 \mathrm{mmol}, 0.33 \mathrm{M}$ in THF, 1.25 equiv) was added dropwise and the reaction mixture was stirred for $2 \mathrm{~h}$ at $50{ }^{\circ} \mathrm{C}$. The reaction was quenched with a saturated aqueous $\mathrm{NH}_{4} \mathrm{Cl}$ solution and the aqueous layer was extracted with EtOAc $(3 \times 75 \mathrm{~mL})$. The combined organic layers were dried over $\mathrm{MgSO}_{4}$, filtered and concentrated under reduced pressure. The crude product was purified by flash column chromatography $\left(\mathrm{SiO}_{2}, i\right.$-hexane $:$ EtOAc $=99: 1, \mathrm{R}_{\mathrm{f}}=$ $0.26)$ yielding the product $\mathbf{5 n}(194 \mathrm{mg}, 0.79 \mathrm{mmol}, 79 \%)$ as a white solid. 
M.p.: $76.3{ }^{\circ} \mathrm{C}$

${ }^{1}$ H-NMR $\left(600 \mathrm{MHz}, \mathbf{C D C l}_{3}, \mathbf{p p m}\right) \delta=7.77(\mathrm{~d}, J=9.0 \mathrm{~Hz}, 2 \mathrm{H}), 7.63(\mathrm{dd}, J=7.5,1.4 \mathrm{~Hz}, 1 \mathrm{H}), 7.58-$ $7.54(\mathrm{~m}, 1 \mathrm{H}), 7.10-7.02(\mathrm{~m}, 1 \mathrm{H}), 6.95(\mathrm{~d}, J=8.9 \mathrm{~Hz}, 2 \mathrm{H}), 3.87(\mathrm{~s}, 3 \mathrm{H}), 2.32(\mathrm{~s}, 3 \mathrm{H})$.

${ }^{13}$ C-NMR (150 MHz, CDCl 3 , ppm) $\delta=194.4,163.6\left(\mathrm{~d},{ }^{1} J(\mathrm{C}, \mathrm{F})=251 \mathrm{~Hz}\right), 163.1,134.1,133.4(\mathrm{~d}$, $\left.{ }^{4} J(\mathrm{C}, \mathrm{F})=3.6 \mathrm{~Hz}\right), 132.4,131.5,130.2,129.6\left(\mathrm{~d},{ }^{3} J(\mathrm{C}, \mathrm{F})=9.0 \mathrm{~Hz}\right), 125.1\left(\mathrm{~d},{ }^{2} J(\mathrm{C}, \mathrm{F})=18 \mathrm{~Hz}\right), 114.8(\mathrm{~d}$, $\left.{ }^{2} J(C, F)=22.9 \mathrm{~Hz}\right), 113.6,55.5,14.5$.

${ }^{19}$ F-NMR (376 MHz, CDCl 3, ppm) $\delta=-111.2$.

IR $\left(A T R, \mathbf{c m}^{-1}\right) \tilde{v}=3071,2959,2934,2842,1711,1644,1596,1587,1511,1464,1441,1419,1318$, 1297, 1255, 1236, 1193, 1167, 1130, 1111, 1019, 964, 902, 873, 846, 818, 795, 766, 754, 685.

MS (EI, 70 eV, \%) m/z = 245 (10), 244 (42), 137 (18), 136 (10), 135 (100), 109 (11), 77 (10).

HRMS (EI, 70 eV) m/z: calc. for $\mathbf{C}_{15} \mathbf{H}_{13} \mathbf{F} \mathbf{O}_{2}$ : 244.0900; found 244.0893 .

Preparation of (4-chlorophenyl)(4-methoxyphenyl)methanone (5o)

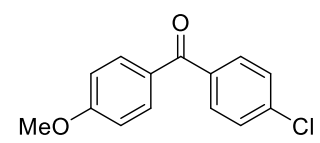

Based on TP4, $\mathrm{FeCl}_{2}$ (6.34 mg, $0.05 \mathrm{mmol}, 5.0 \mathrm{~mol} \%$ ), 4-chlorobenzoyl chloride (2a, $175 \mathrm{mg}, 0.13 \mathrm{~mL}$, $1.00 \mathrm{mmol}, 1.00$ equiv) and freshly distilled THF (1.0 mL) were used. The (4-methoxyphenyl)zinc(II) chloride (4f, $3.50 \mathrm{~mL}, 1.25 \mathrm{mmol}, 0.36 \mathrm{M}$ in THF, 1.25 equiv) was added dropwise and the reaction mixture was stirred for $3 \mathrm{~h}$ at $50{ }^{\circ} \mathrm{C}$. The reaction was quenched with a saturated aqueous $\mathrm{NH}_{4} \mathrm{Cl}$ solution and the aqueous layer was extracted with EtOAc $(3 \times 75 \mathrm{~mL})$. The combined organic layers were dried over $\mathrm{MgSO}_{4}$, filtered and concentrated under reduced pressure. Purification of the crude product by flash column chromatography $\left(\mathrm{SiO}_{2}, i\right.$-hexane $:$ EtOAc $\left.=19: 1, \mathrm{R}_{\mathrm{f}}=0.30\right)$ afforded product $\mathbf{5 o}(182 \mathrm{mg}$, $0.74 \mathrm{mmol}, 74 \%$ ) as a yellow solid.

M.p.: $118.6^{\circ} \mathrm{C}$

${ }^{1} \mathrm{H}-\mathrm{NMR}\left(400 \mathrm{MHz}, \mathbf{C D C l}_{3}\right.$, ppm) $\delta=7.73(\mathrm{~d}, J=8.9 \mathrm{~Hz}, 2 \mathrm{H}), 7.64(\mathrm{~d}, J=8.6 \mathrm{~Hz}, 2 \mathrm{H}), 7.38(\mathrm{~d}, J=$ $8.6 \mathrm{~Hz}, 2 \mathrm{H}), 6.90(\mathrm{~d}, J=8.9 \mathrm{~Hz}, 2 \mathrm{H}), 3.82(\mathrm{~s}, 3 \mathrm{H})$.

${ }^{13} \mathbf{C}-\mathrm{NMR}\left(\mathbf{1 0 0} \mathbf{M H z}, \mathbf{C D C l}_{\mathbf{3}}, \mathbf{p p m}\right) \delta=194.2,163.4,138.3,136.6,132.4,131.2,129.8,128.5,113.7$, 55.5 . 
IR $\left(\mathbf{A T R}, \mathbf{c m}^{-1}\right) \tilde{\mathrm{v}}=3014,2934,2841,1722,1637,1600,1588,1509,1461,1414,1397,1299,1283$, 1245, 1170, 1147, 1086, 1062, 1028, 1013, 966, 950, 925, 853, 834, 759, 736, 722, 678.

MS (EI, 70 eV, \%) m/z = 248 (32), 247 (14), 246 (99), 211 (16), 138 (26), 136 (22), 135 (100), 111 (22), 92 (18), 77 (22), 75 (12).

HRMS (EI, $70 \mathrm{eV}$ ) m/z: calc. for $\mathbf{C}_{14} \mathbf{H}_{11} \mathbf{C l O}_{2}$ : 246.0448; found 246.0438 .

\section{Preparation of (4-(tert-butyl)phenyl)(4-methoxyphenyl)methanone (5p)}

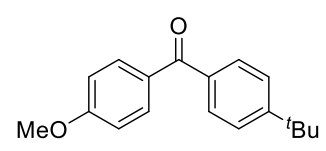

Based on TP4, $\mathrm{FeCl}_{2}$ (6.34 mg, $0.05 \mathrm{mmol}, 5.0 \mathrm{~mol} \%$ ), 4-tert-butylbenzoyl chloride (2b, $197 \mathrm{mg}$, $0.20 \mathrm{~mL}, 1.00 \mathrm{mmol}, 1.00$ equiv) and freshly distilled THF (1.0 mL) were used. The (4-methoxyphenyl)zinc(II) chloride (4f, $3.50 \mathrm{~mL}, 1.25 \mathrm{mmol}, 0.36 \mathrm{M}$ in THF, 1.25 equiv) was added dropwise and the reaction mixture was stirred for $3 \mathrm{~h}$ at $50{ }^{\circ} \mathrm{C}$. The reaction was quenched with a saturated aqueous $\mathrm{NH}_{4} \mathrm{Cl}$ solution and the aqueous layer was extracted with EtOAc $(3 \times 75 \mathrm{~mL})$. The combined organic layers were dried over $\mathrm{MgSO}_{4}$, filtered and concentrated under reduced pressure. Purification of the crude product by flash column chromatography $\left(\mathrm{SiO}_{2}, i\right.$-hexane $:$ EtOAc $\left.=19: 1, \mathrm{R}_{\mathrm{f}}=0.28\right)$ furnished product 5p (219 mg, $0.82 \mathrm{mmol}, 82 \%)$ as a colourless oil.

${ }^{1} \mathrm{H}-\mathrm{NMR}\left(400 \mathrm{MHz}, \mathbf{C D C l}_{3}\right.$, ppm) $\delta=7.77(\mathrm{~d}, J=8.9 \mathrm{~Hz}, 2 \mathrm{H}), 7.65(\mathrm{~d}, J=8.6 \mathrm{~Hz}, 2 \mathrm{H}), 7.42(\mathrm{~d}, J=$ $8.6 \mathrm{~Hz}, 2 \mathrm{H}), 6.89$ (d, J=8.9 Hz, 2H), 3.80 (s, 3H), 1.30 (s, 9H).

${ }^{13}$ C-NMR (100 MHz, $\mathbf{C D C l}_{3}$, ppm) $\delta=195.2,163.1,155.6,135.5,132.5,130.5,129.8,127.0,113.5$, $55.5,35.1,31.2$.

IR $\left(A T R, \mathbf{c m}^{-1}\right) \tilde{v}=2962,1649,1600,1574,1509,1462,1442,1418,1364,1314,1305,1281,1252$, $1172,1151,1104,1028,951,929,853,836,798,772,728,687$.

MS (EI, 70 eV, \%) m/z = 269 (13), 268 (57), 254 (27), 253 (100), 161 (14), 135 (65), 77 (12), 44 (20).

HRMS (EI, $70 \mathbf{~ e V}$ ) m/z: calc. for $\mathbf{C}_{18} \mathbf{H}_{20} \mathbf{O}_{2}$ : 268.1463; found 268.1447 . 
Preparation of (4-fluorophenyl)(4-methoxyphenyl)methanone (5q)

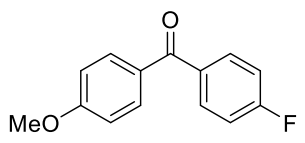

According to TP4, $\mathrm{FeCl}_{2}$ (6.34 mg, $0.05 \mathrm{mmol}, 5.0 \mathrm{~mol} \%$ ), 4-fluorobenzoyl chloride (2c, $159 \mathrm{mg}$, $0.12 \mathrm{~mL}, 1.00 \mathrm{mmol}, 1.00$ equiv) and freshly distilled THF (1.0 mL) were used. The (4-methoxyphenyl)zinc(II) chloride (4f, $3.50 \mathrm{~mL}, 1.25 \mathrm{mmol}, 0.36 \mathrm{M}$ in THF, 1.25 equiv) was added dropwise and the reaction mixture was stirred for $3 \mathrm{~h}$ at $50{ }^{\circ} \mathrm{C}$. The reaction was quenched with a saturated aqueous $\mathrm{NH}_{4} \mathrm{Cl}$ solution and the aqueous layer was extracted with EtOAc $(3 \times 75 \mathrm{~mL})$. The combined organic layers were dried over $\mathrm{MgSO}_{4}$, filtered and concentrated under reduced pressure. The crude product was purified by flash column chromatography $\left(\mathrm{SiO}_{2}, i\right.$-hexane $:$ EtOAc $\left.=19: 1, \mathrm{R}_{\mathrm{f}}=0.15\right)$ leading to the product 5q (189 $\mathrm{mg}, 0.82 \mathrm{mmol}, 82 \%)$ as a yellow solid.

M.p.: $92.4{ }^{\circ} \mathrm{C}$

${ }^{1} \mathbf{H}-\mathrm{NMR}\left(\mathbf{4 0 0} \mathbf{M H z}, \mathbf{C D C l}_{\mathbf{3}}, \mathbf{p p m}\right) \delta=7.73(\mathrm{dt}, J=9.0,2.7 \mathrm{~Hz}, 4 \mathrm{H}), 7.08(\mathrm{t}, J=8.7 \mathrm{~Hz}, 2 \mathrm{H}), 6.90(\mathrm{~d}$, $J=8.9 \mathrm{~Hz}, 2 \mathrm{H}), 3.82(\mathrm{~s}, 3 \mathrm{H})$.

${ }^{13} \mathrm{C}-\mathrm{NMR}\left(100 \mathrm{MHz}, \mathbf{C D C l}_{3}\right.$, ppm) $\delta=194.1,165.1\left(\mathrm{~d},{ }^{1} J(\mathrm{C}, \mathrm{F})=252 \mathrm{~Hz}\right), 163.3,134.5\left(\mathrm{~d},{ }^{4} J(\mathrm{C}, \mathrm{F})=\right.$ $3.1 \mathrm{~Hz}), 132.4,132.3\left(\mathrm{~d},{ }^{3} J(\mathrm{C}, \mathrm{F})=9.0 \mathrm{~Hz}\right), 127.0,115.3\left(\mathrm{~d},{ }^{2} J(\mathrm{C}, \mathrm{F})=22 \mathrm{~Hz}\right), 113.6,55.5$.

${ }^{19} \mathrm{~F}-\mathrm{NMR}\left(376 \mathrm{MHz}, \mathrm{CDCl}_{3}, \mathrm{ppm}\right) \delta=-107.0$.

IR $\left(\mathbf{A T R}, \mathbf{c m}^{-1}\right) \tilde{\mathrm{v}}=2931,2848,1720,1639,1597,1574,1506,1465,1442,1416,1406,1303,1282$, $1247,1226,1174,1148,1116,1028,1013,967,927,856,841,789,763,681$.

MS (EI, 70 eV, \%) m/z = 230 (31), 135 (38), 123 (25), 95 (13), 85 (13), 83 (12), 71 (20), 57 (26), 55 (15), 44 (37), 43 (19).

HRMS (EI, $70 \mathbf{~ e V}$ ) m/z: calc. for $\mathbf{C}_{\mathbf{1 4}} \mathbf{H}_{\mathbf{1 1}} \mathrm{FO}_{\mathbf{2}}$ : 230.0743; found 230.0747 . 
NMR-Spectra of 1-(4-chlorophenyl)-2-phenylethan-1-one (3a) (1)

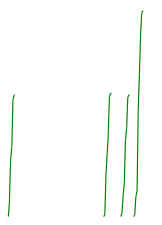<smiles>O=C(Cc1ccccc1)c1ccc(Cl)cc1</smiles>

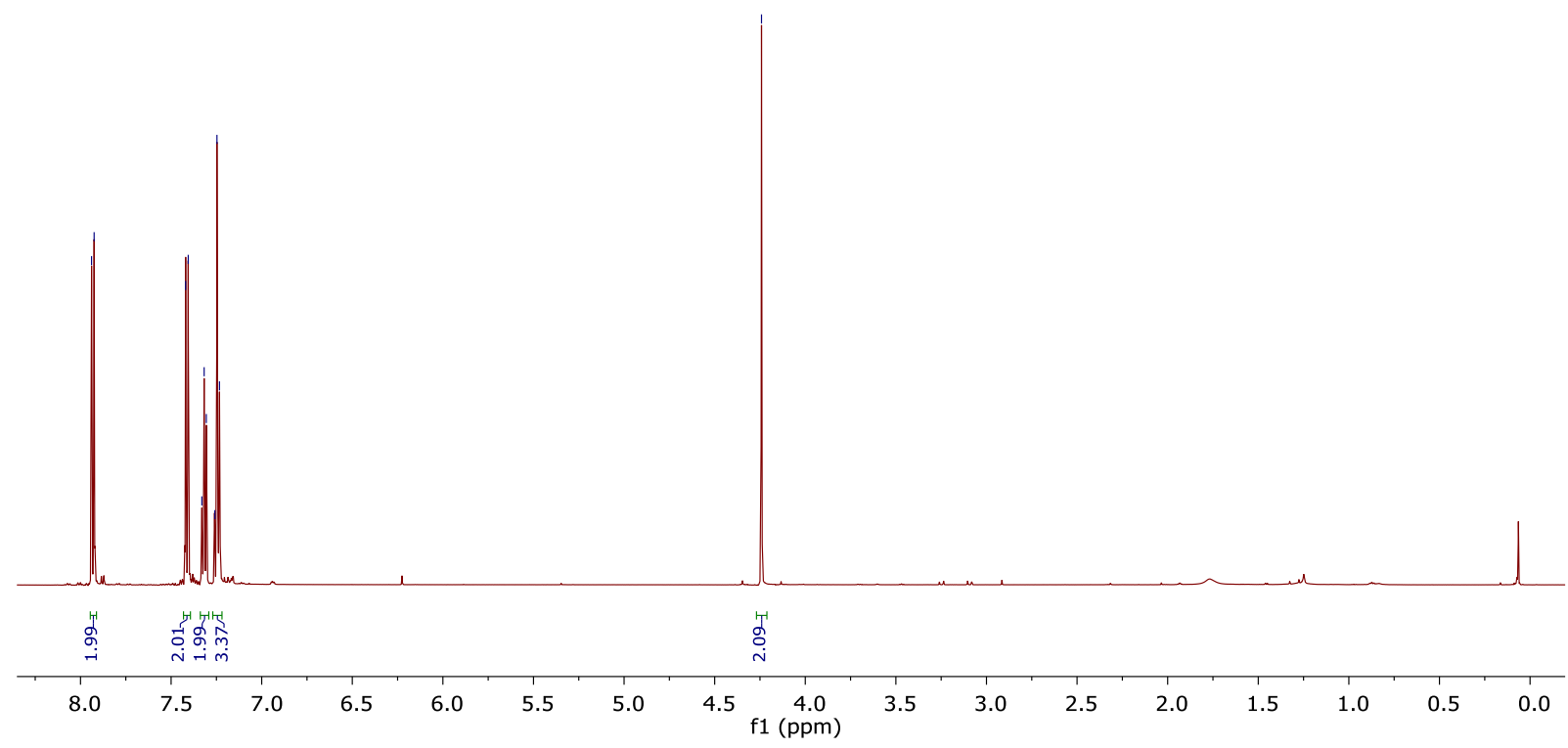

ñ
ڤ్
$\stackrel{1}{।}$ นำㅇำㅇํㅇ용ํㅇ

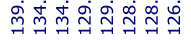
皮
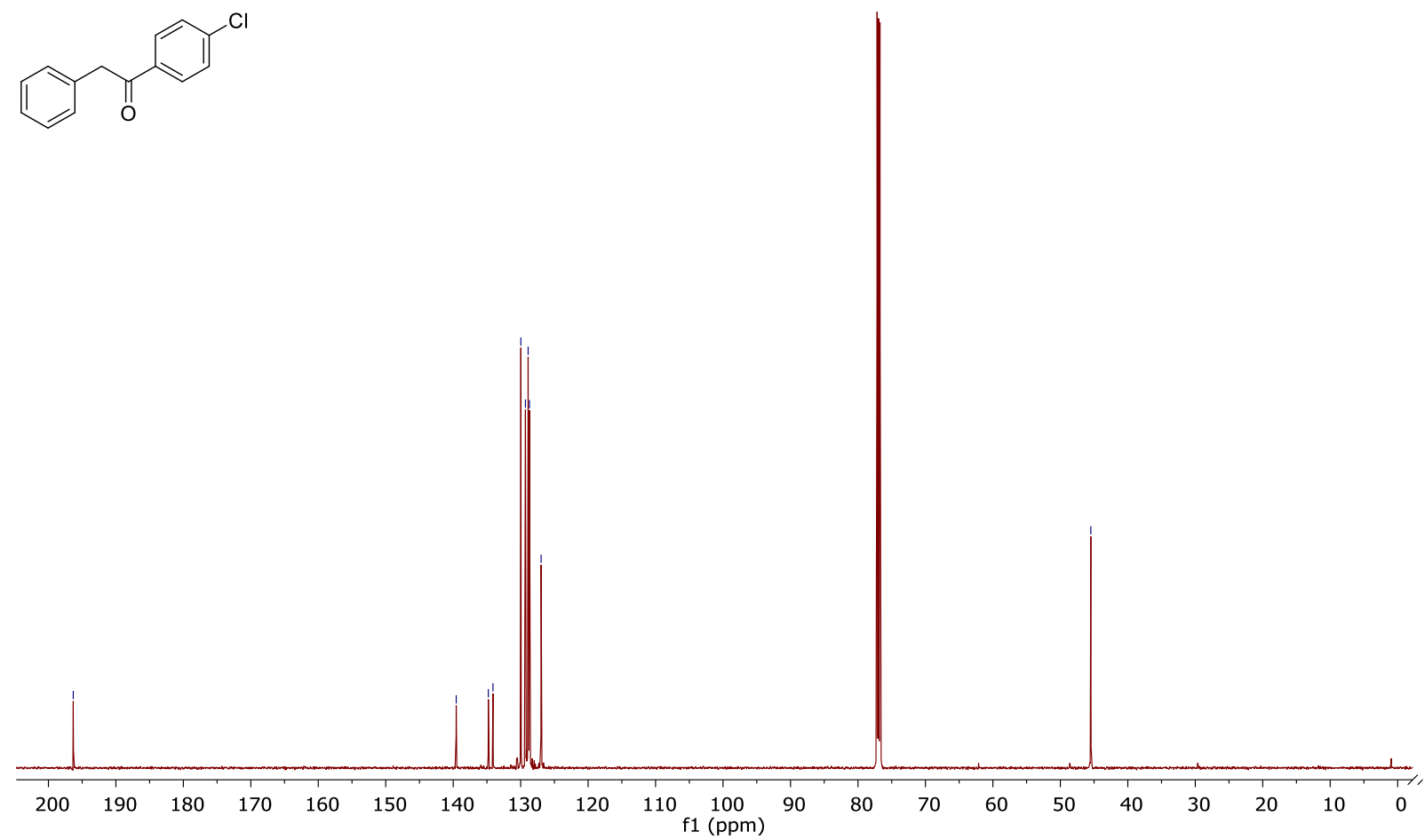
NMR-Spectra of 1-(4-(tert-butyl)phenyl)-2-(3-(trifluoromethyl)phenyl)ethan-1-one (3b)

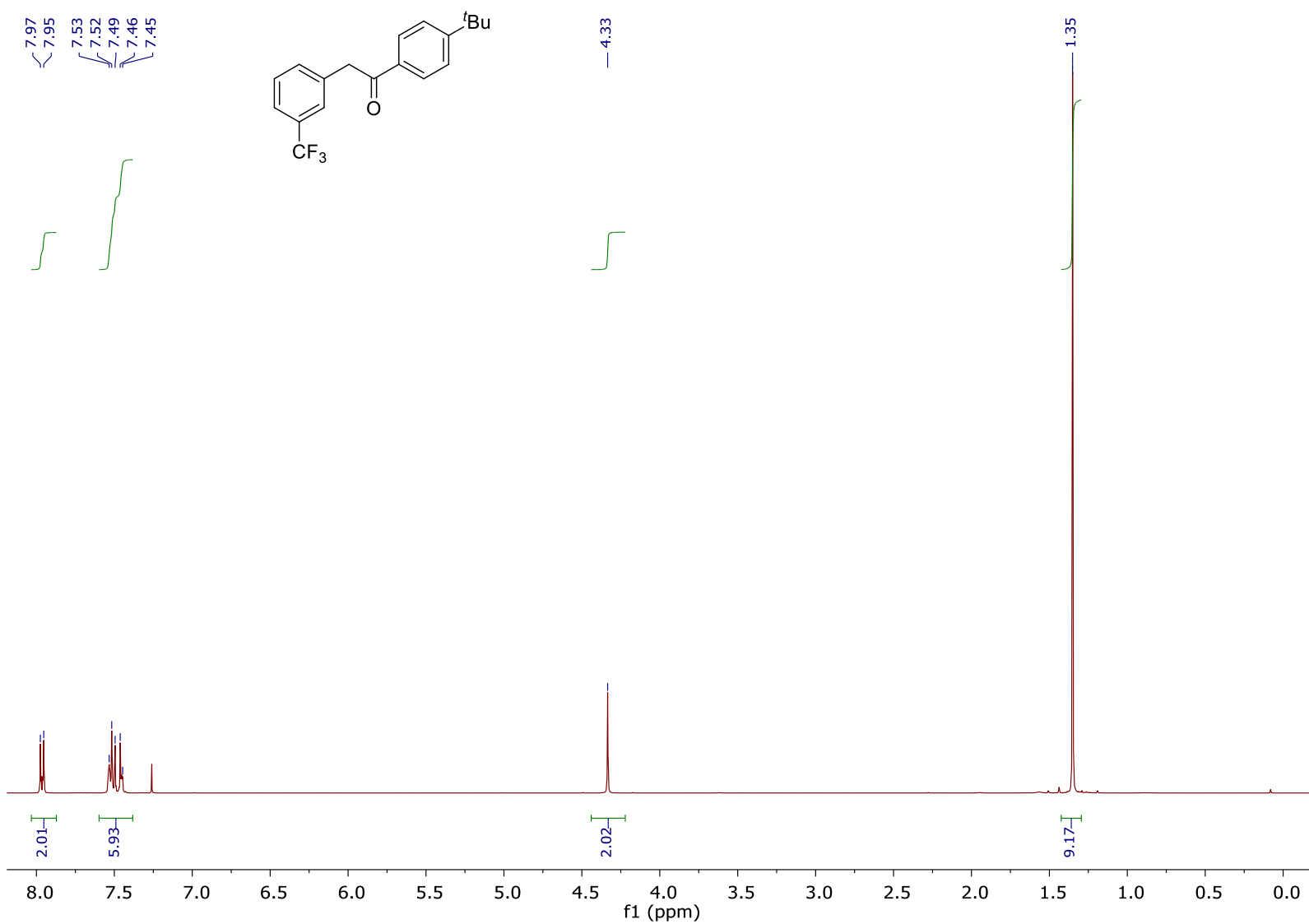

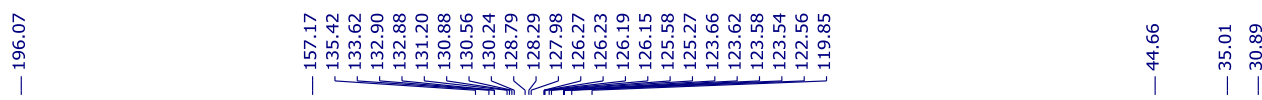
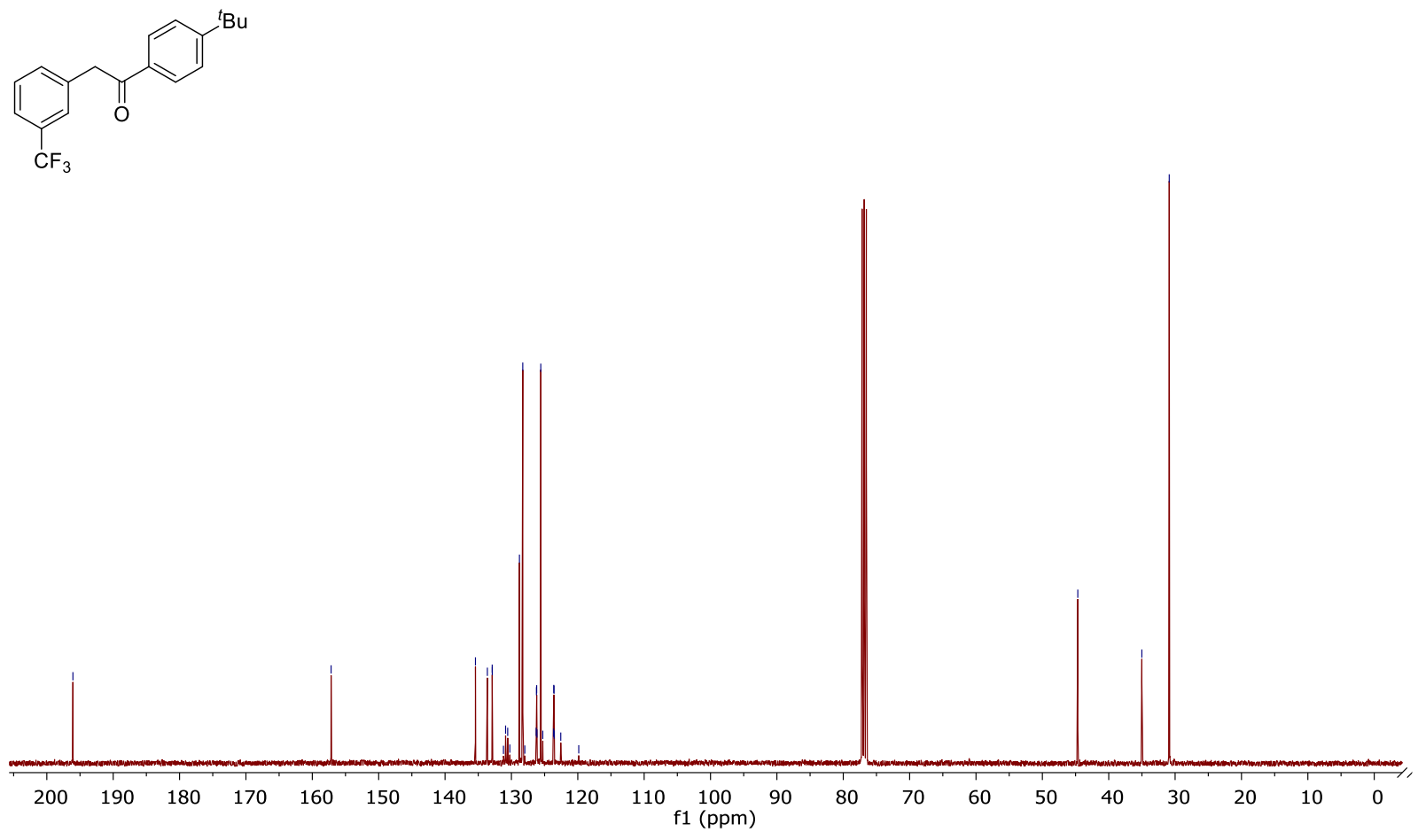


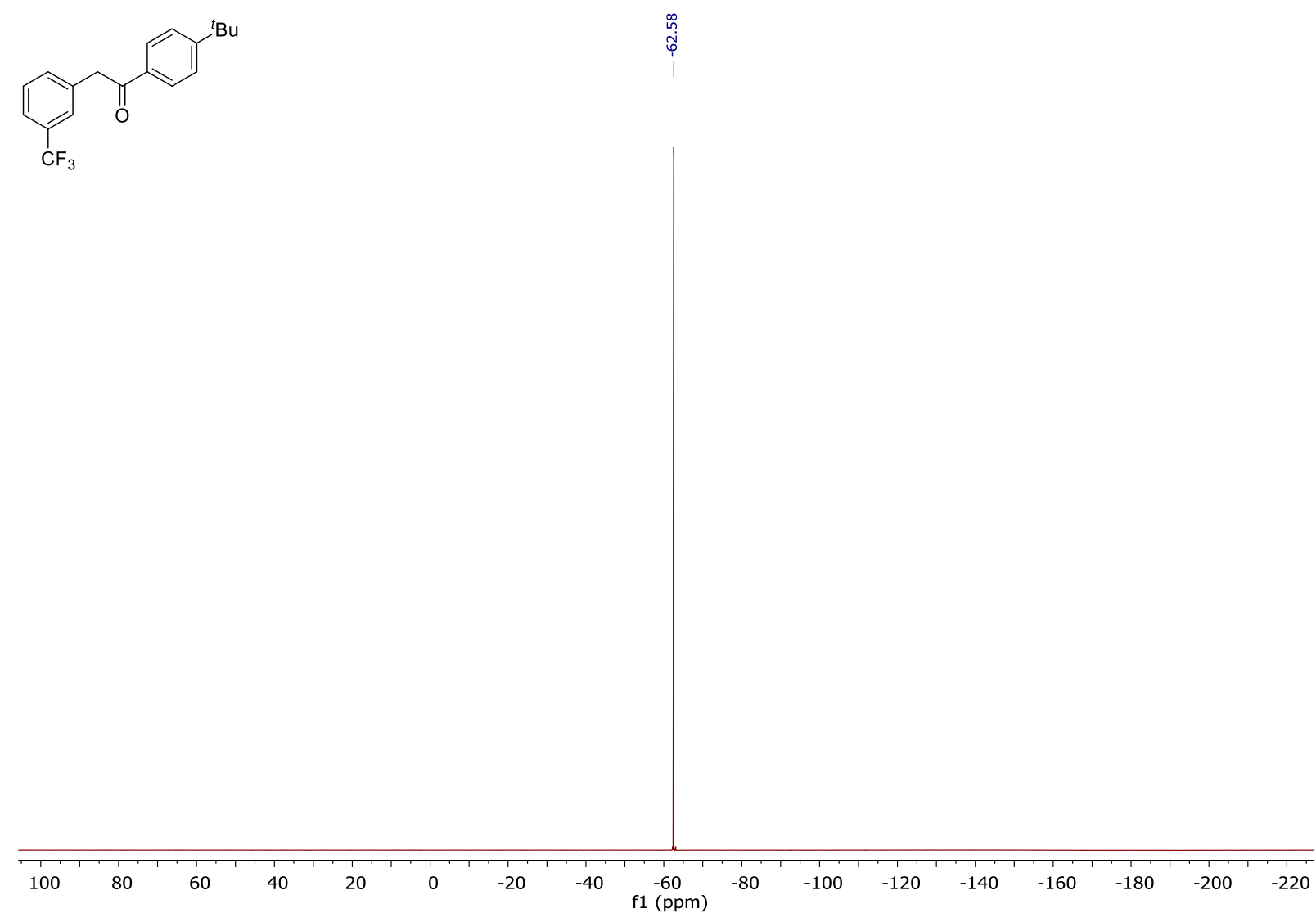

NMR-Spectra of 1-(4-chlorophenyl)-2-(3-(trifluoromethyl)phenyl)ethan-1-one (3c)

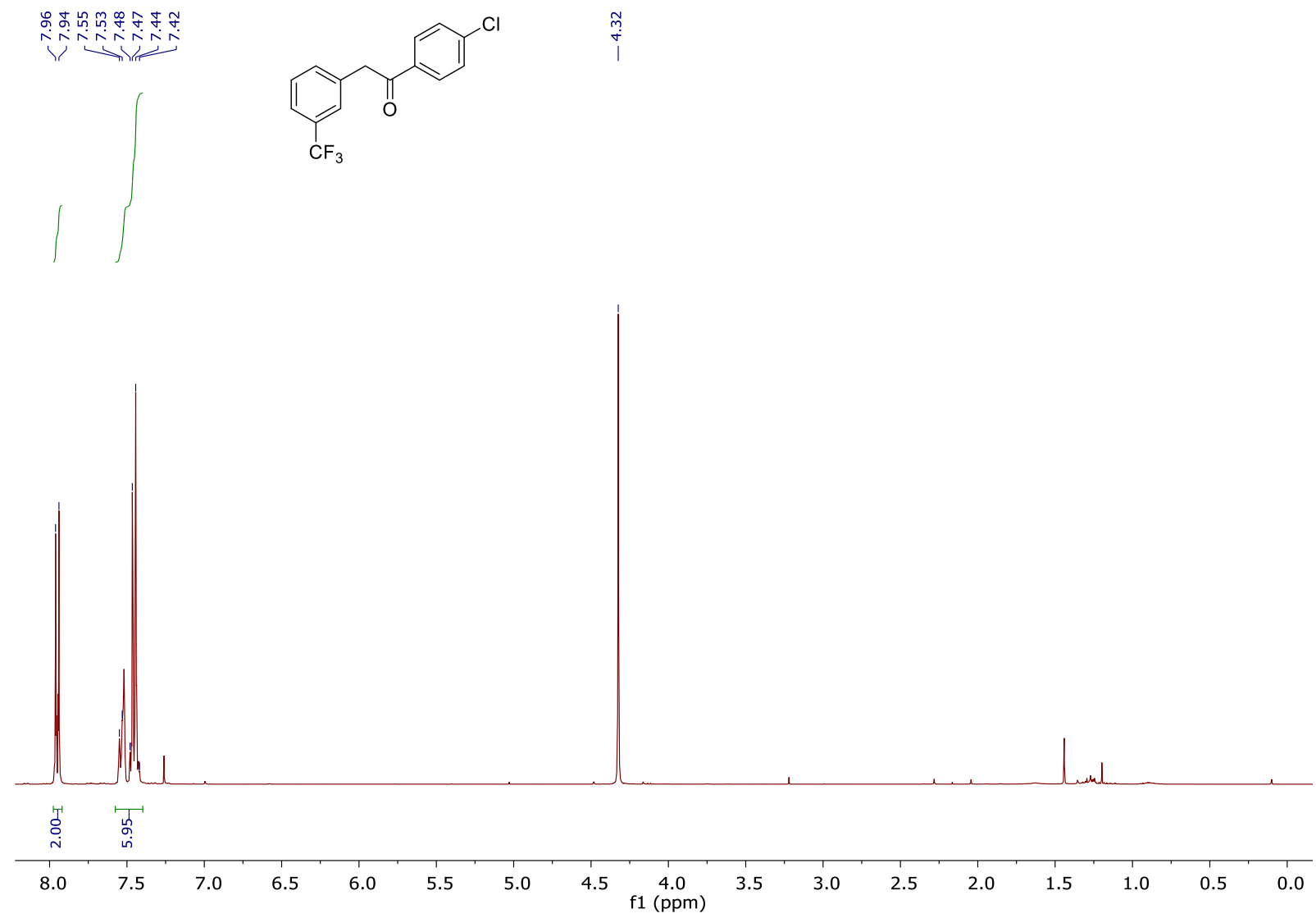



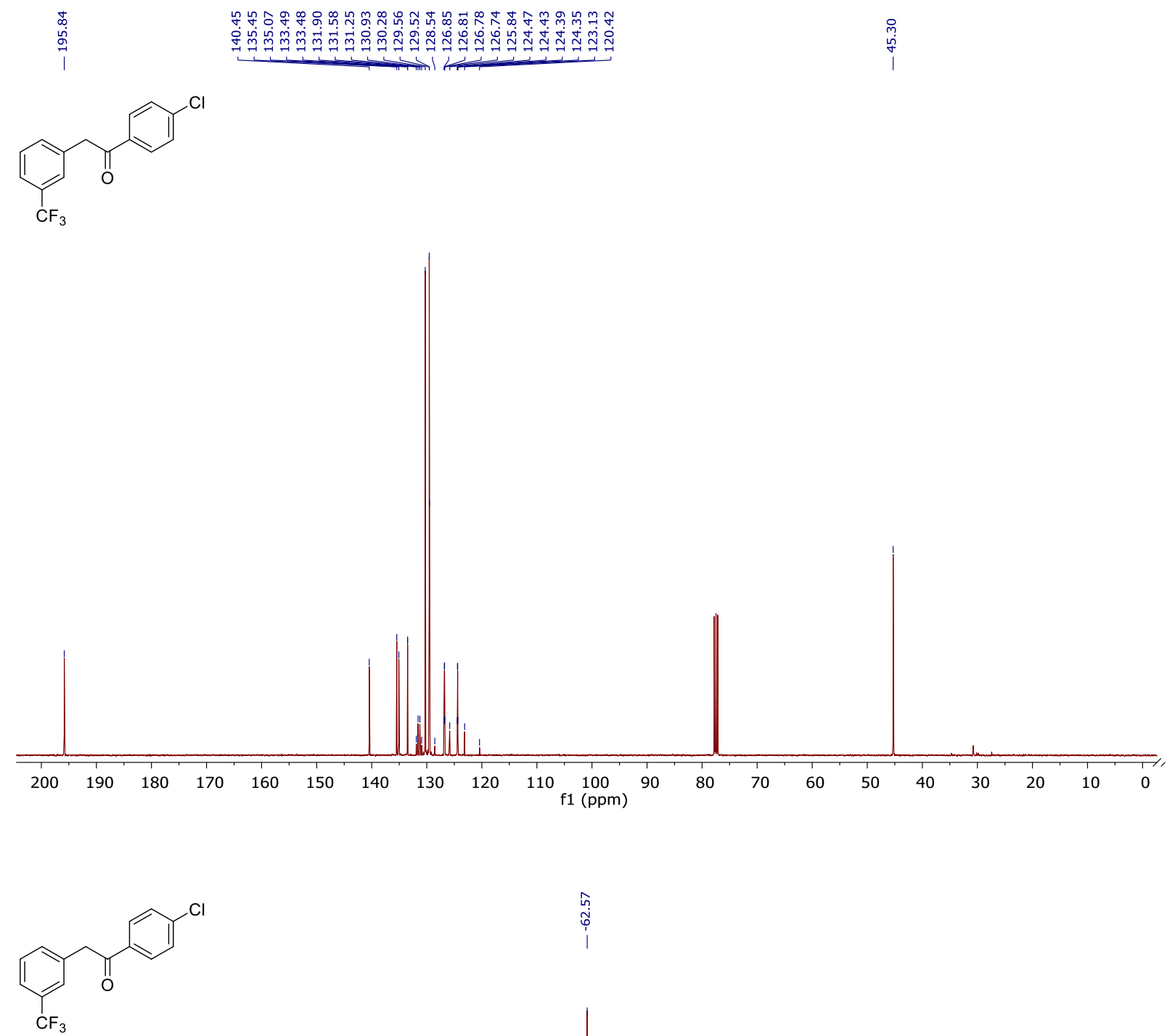
NMR-Spectra of 1-(4-(tert-butyl)phenyl)-2-(3-fluorophenyl)ethan-1-one (3d)

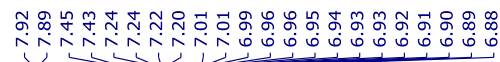
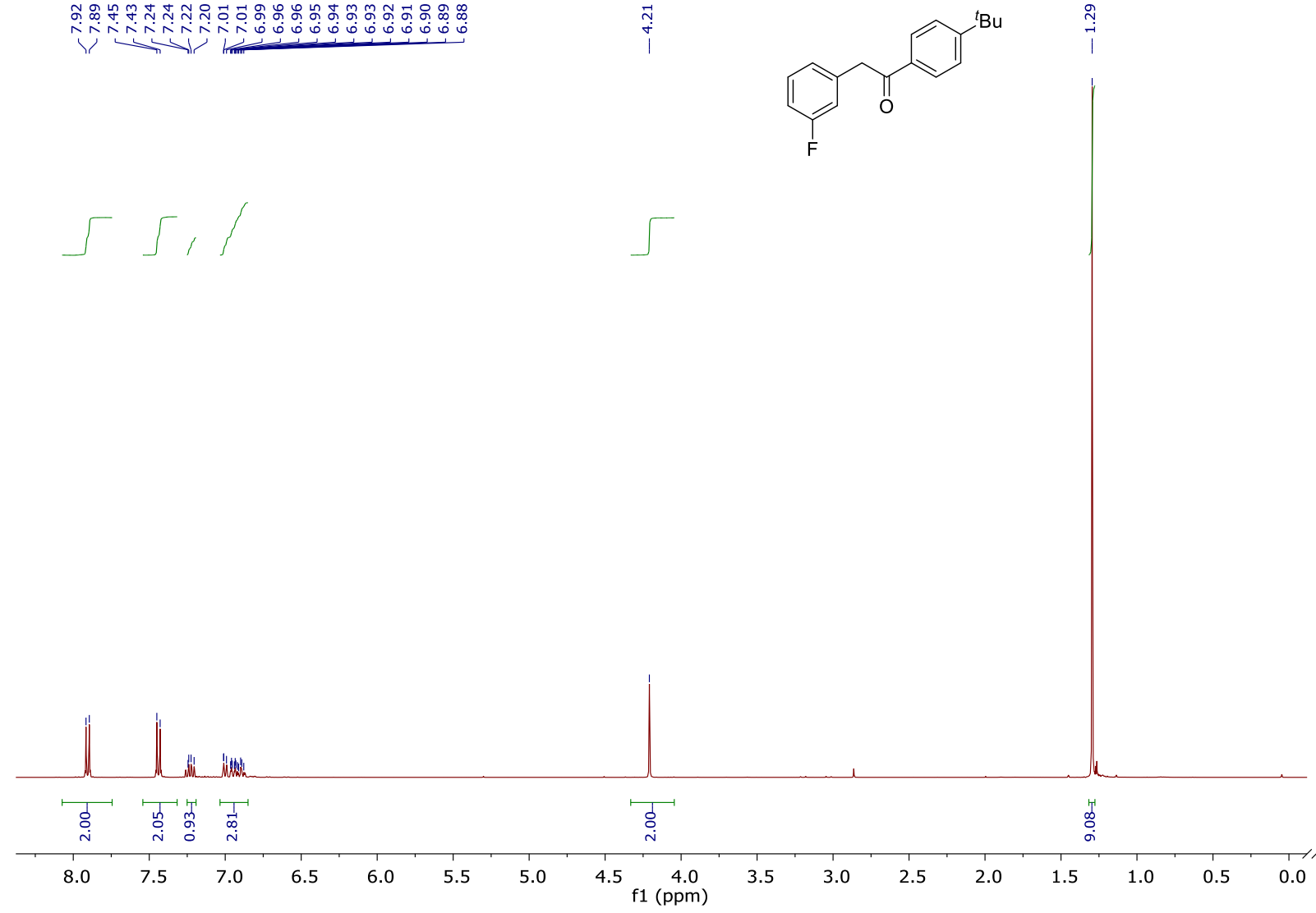

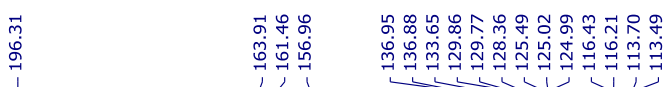<smiles>O=C(Cc1cccc(F)c1)c1ccc(Br)cc1</smiles>

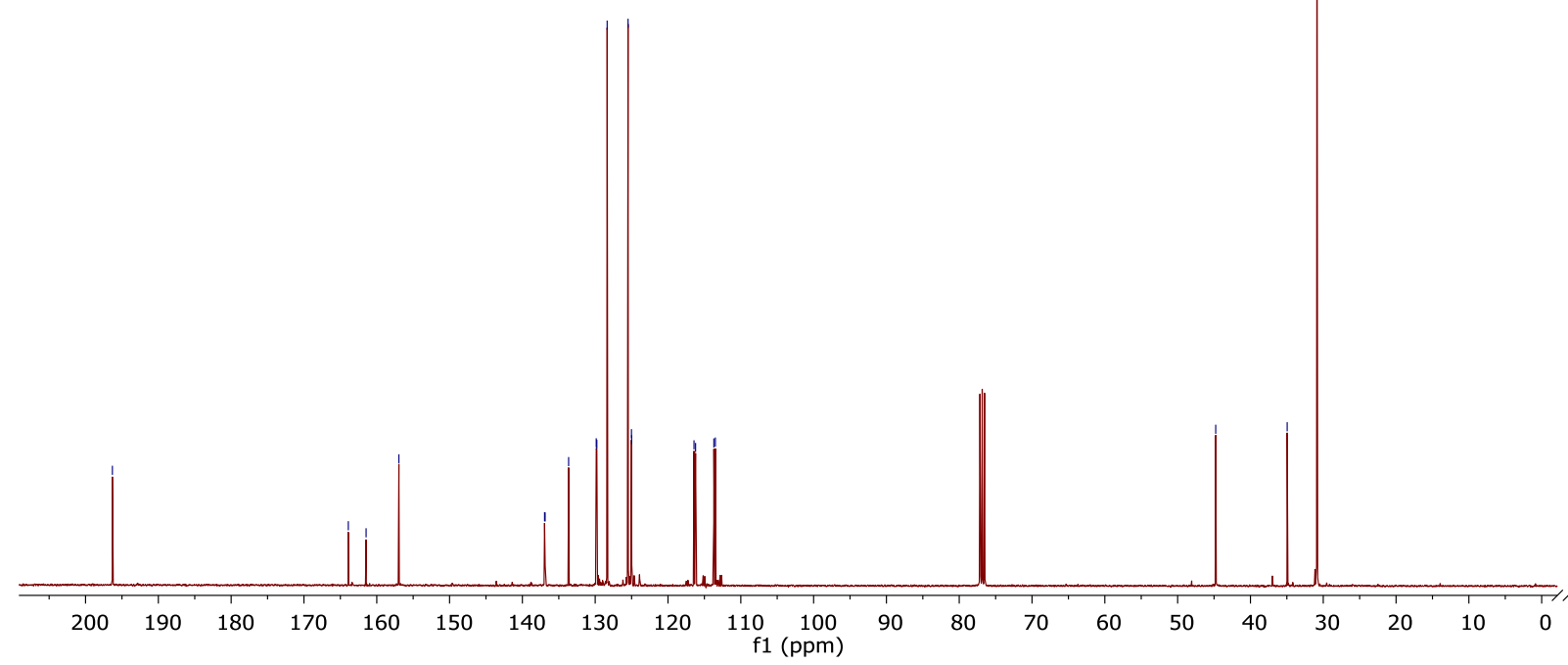




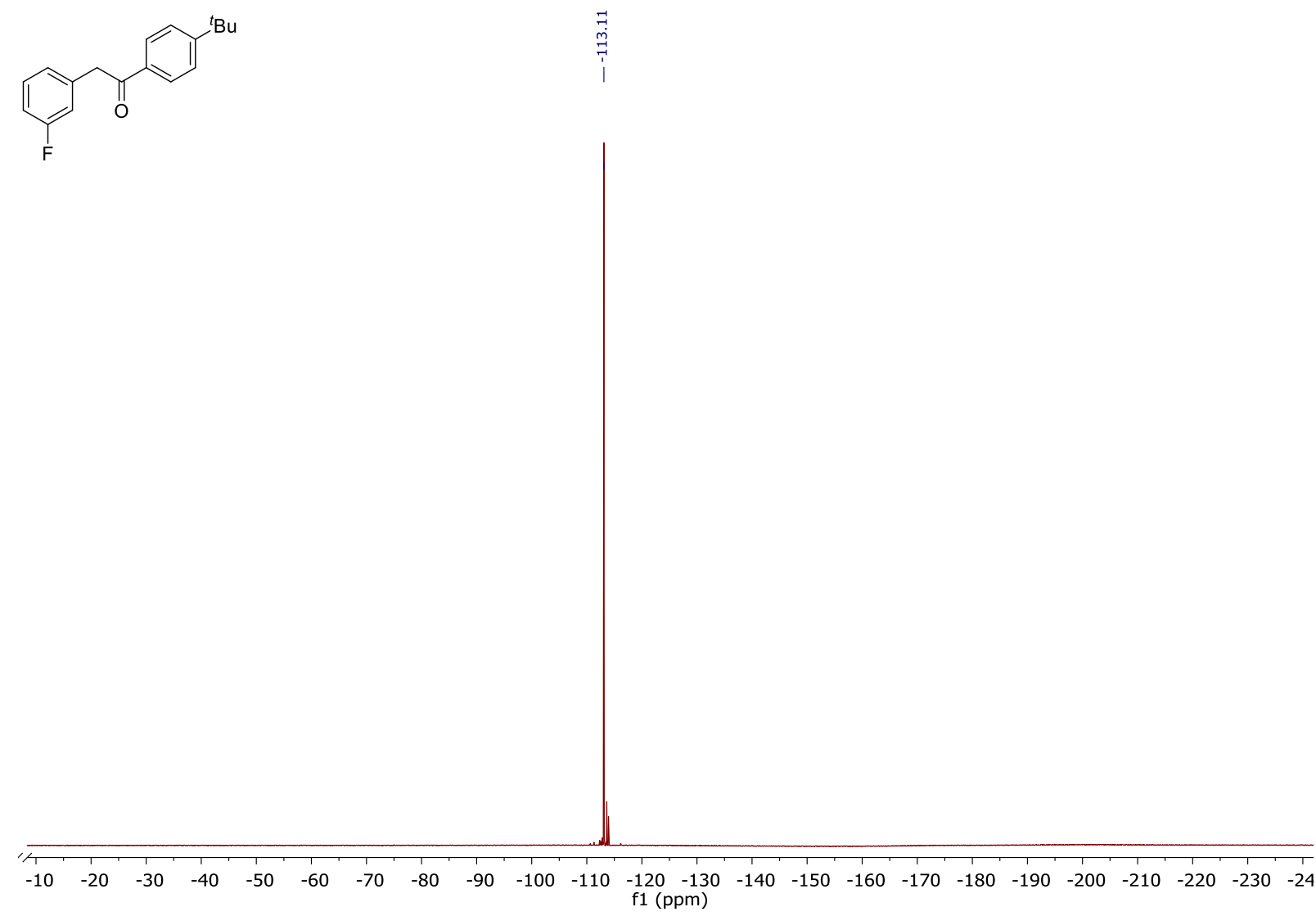

NMR-Spectra of 2-(3-fluorophenyl)-1-(4-fluorophenyl)ethan-1-one (3e)
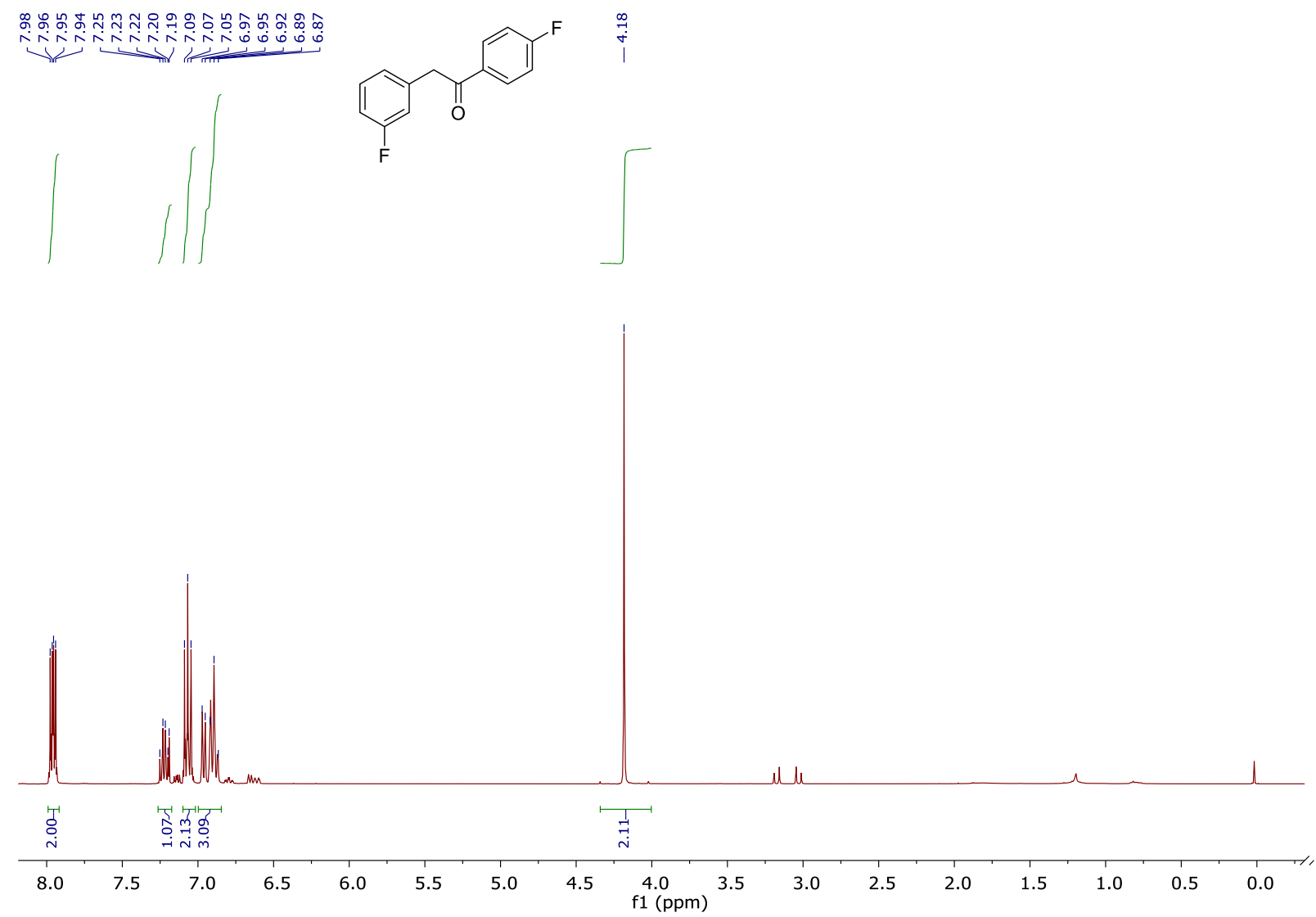

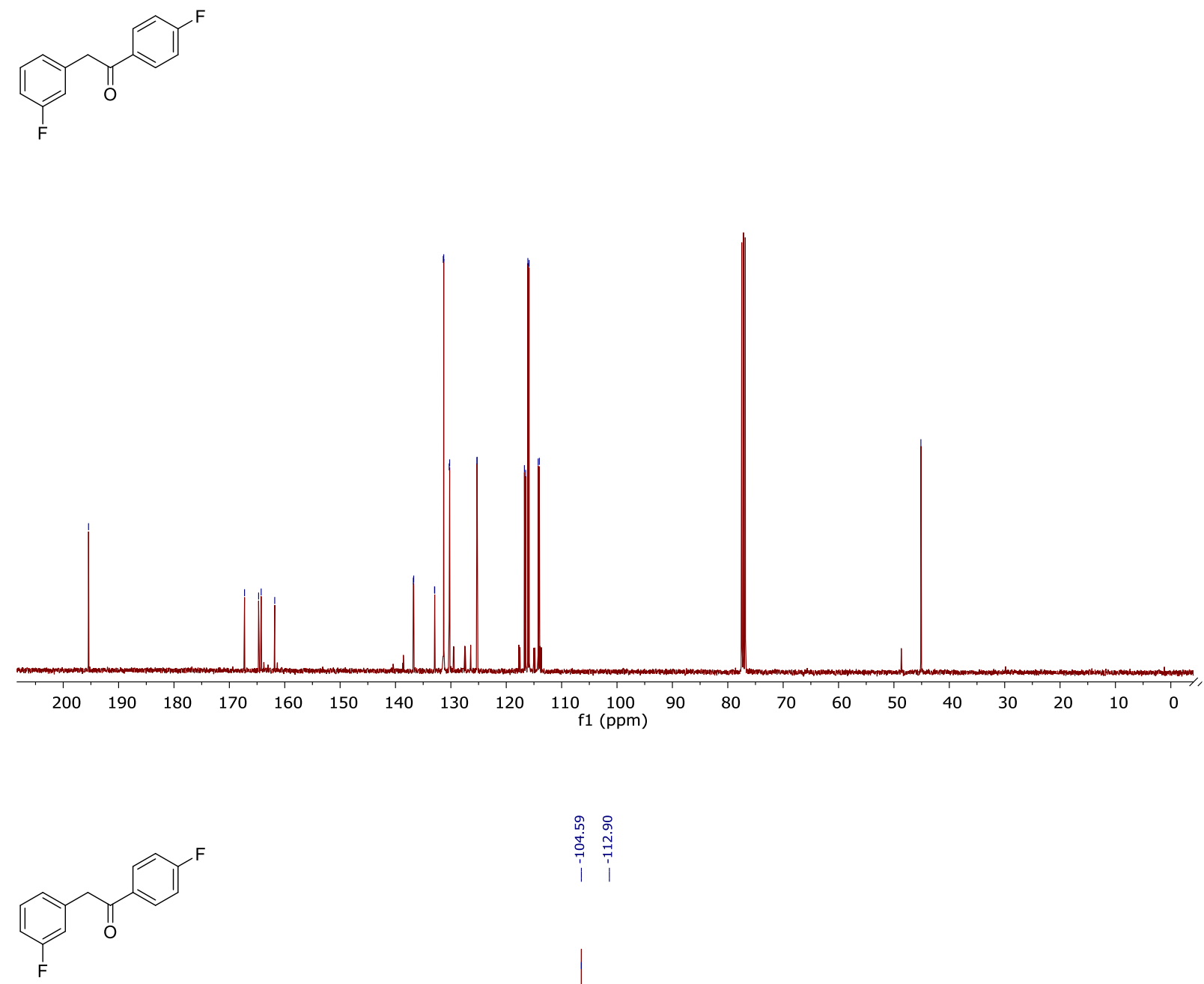

\begin{tabular}{ll}
0 & 8 \\
0 & \multirow{2}{0}{} \\
0 & 7 \\
1 & 7
\end{tabular}

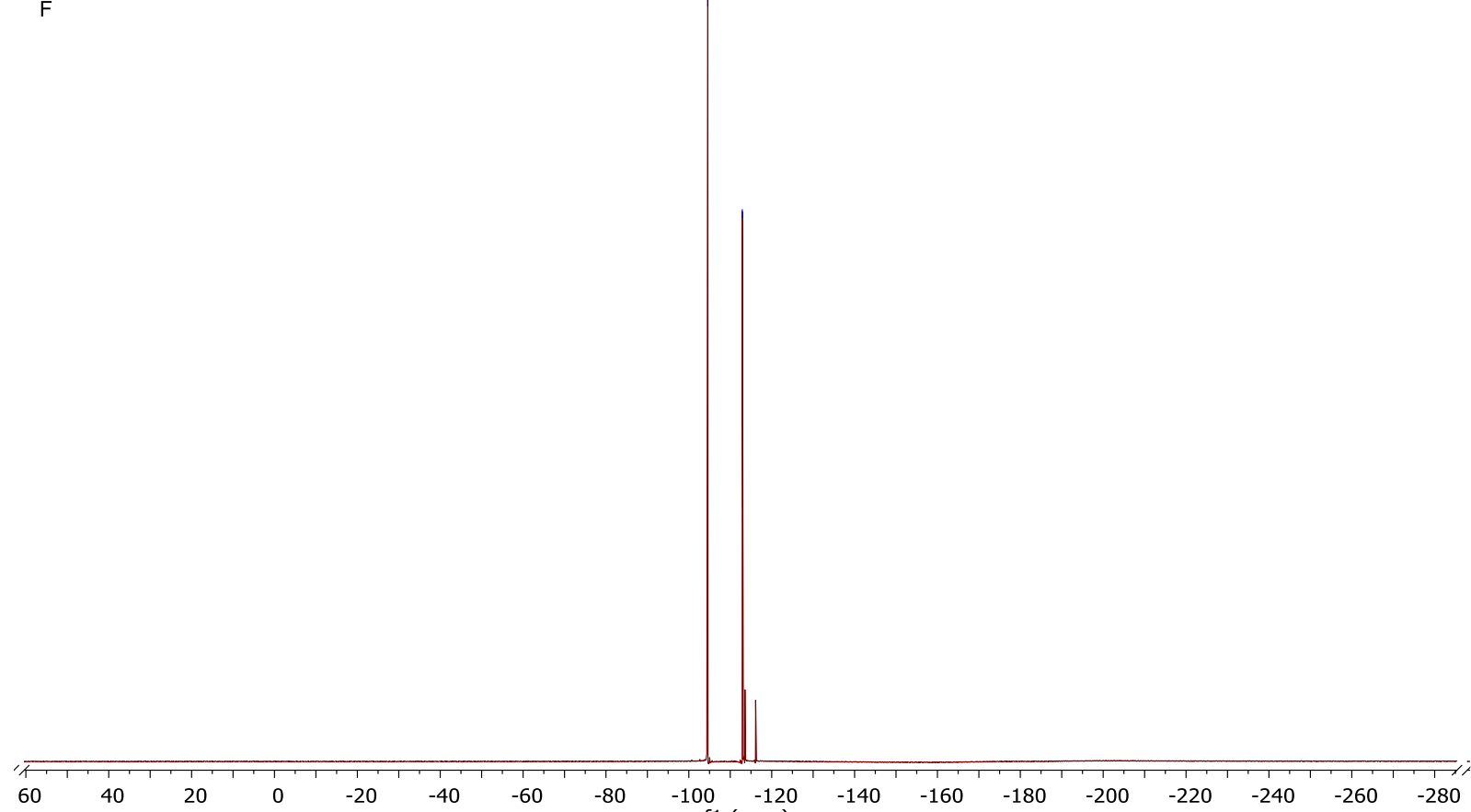


NMR-Spectra of ethyl 3-(2-(4-chlorophenyl)-2-oxoethyl)benzoate (3f)

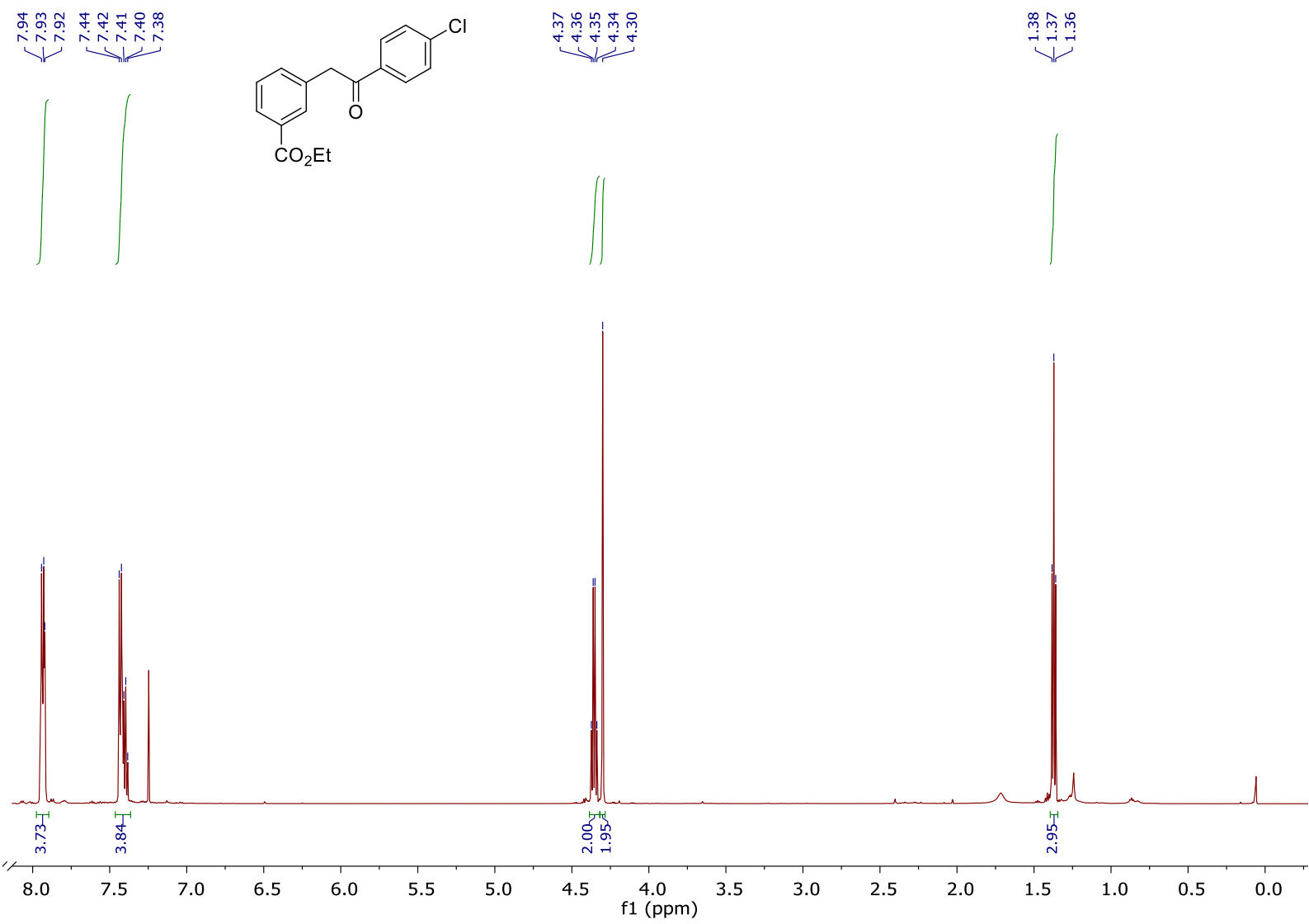

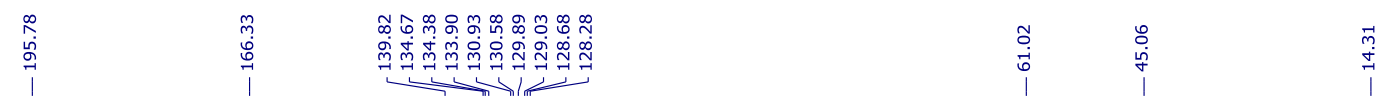
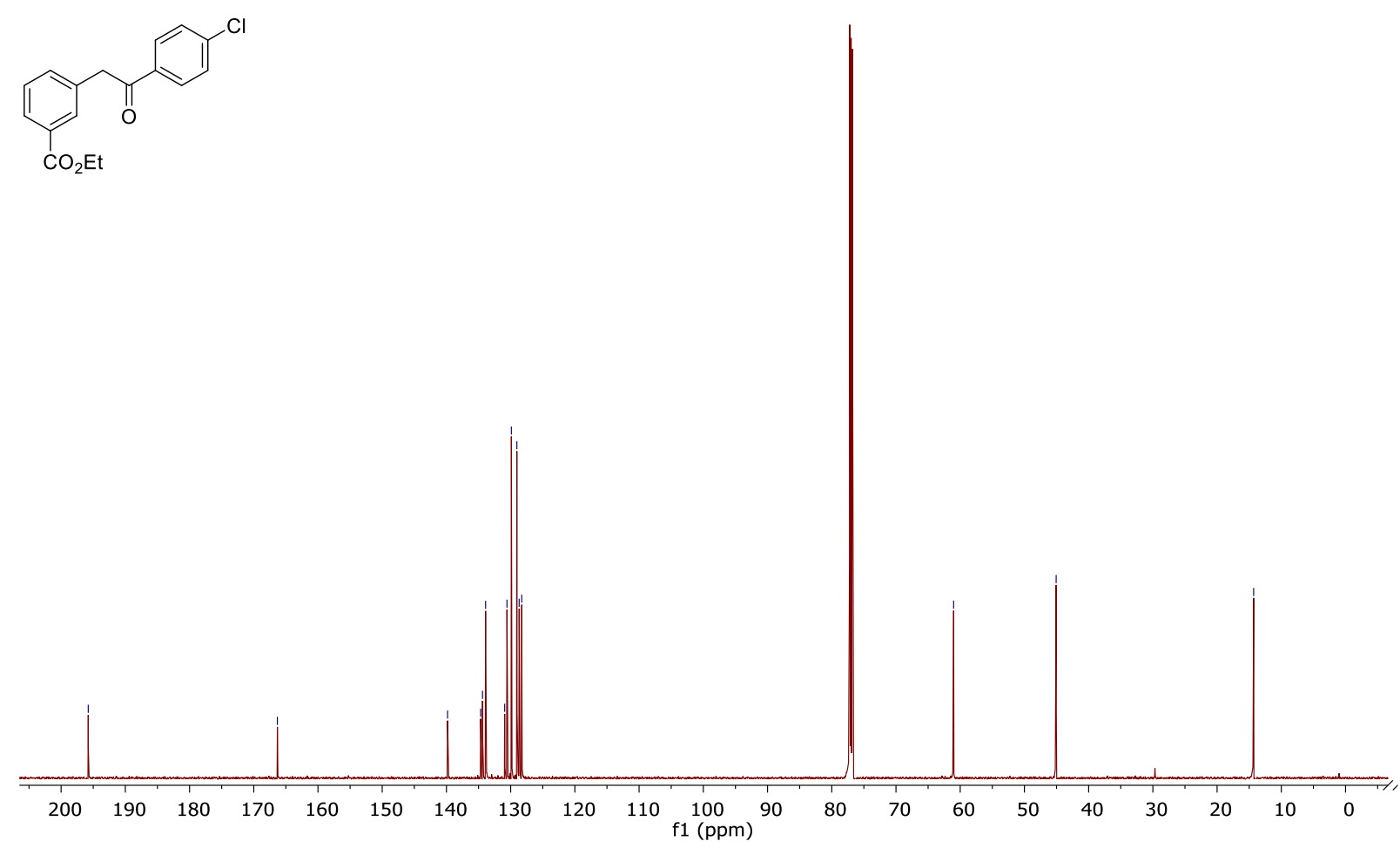
NMR-Spectra of 1-(4-(tert-butyl)phenyl)-2-(4-methoxyphenyl)ethan-1-one (3g)

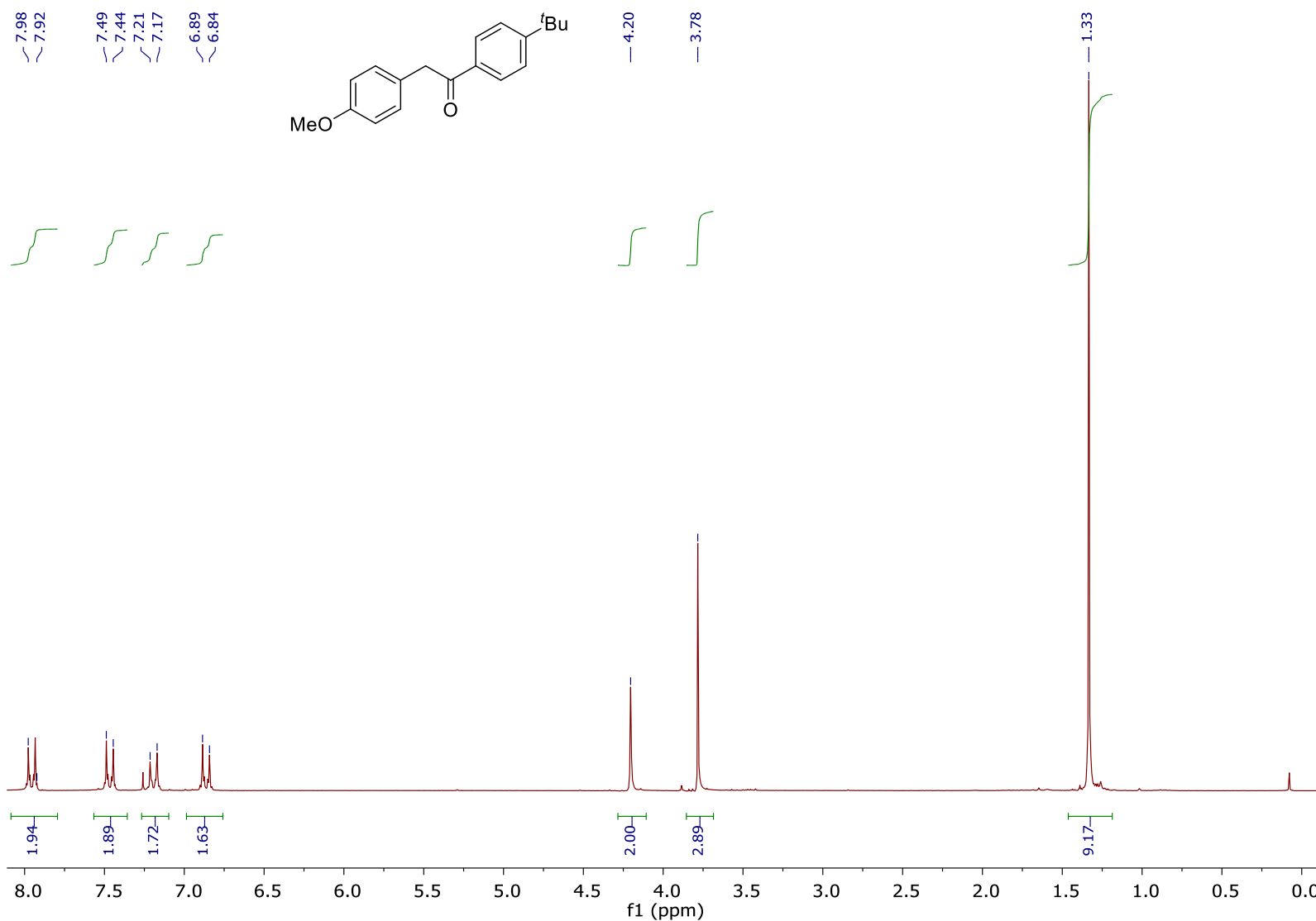

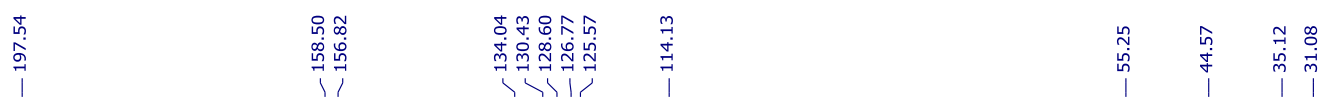<smiles>COc1ccc(CC(=O)c2ccc(Br)cc2)cc1</smiles>

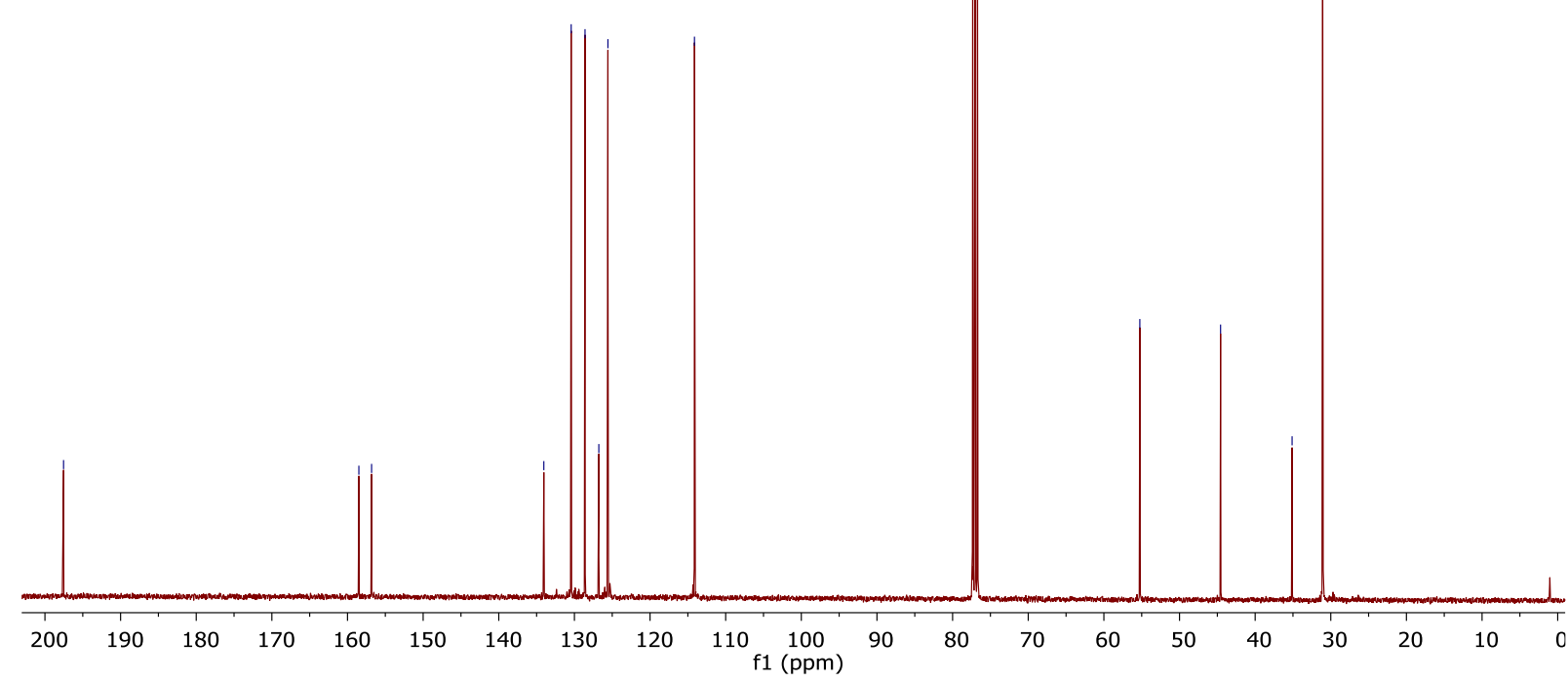


NMR-Spectra of 2-(4-(methylthio)phenyl)-1-(thiophen-2-yl)ethan-1-one (3h)
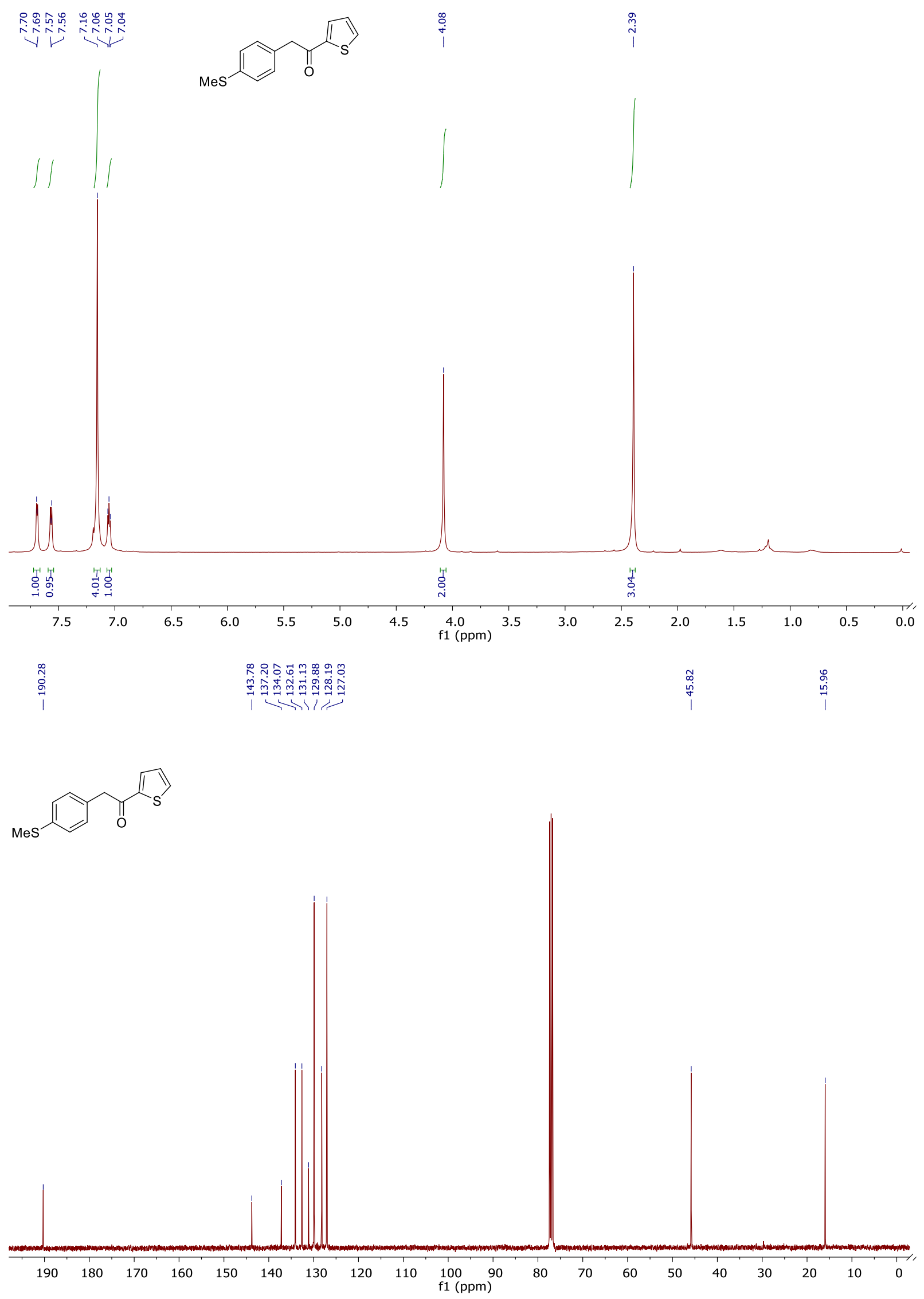

38 
NMR-Spectra of 1-(4-chlorophenyl)-2-phenylpropan-1-one (3i)
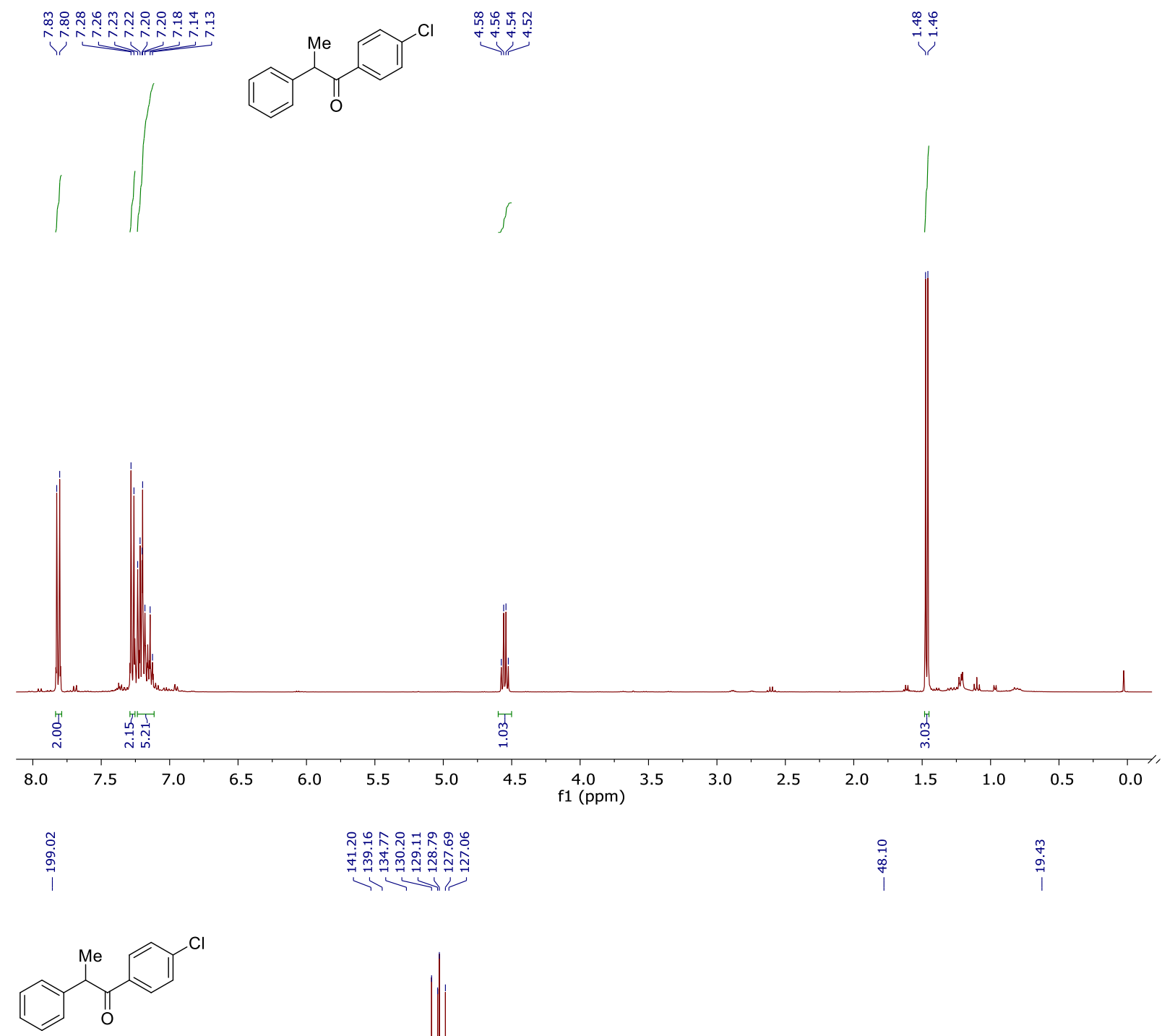

逐

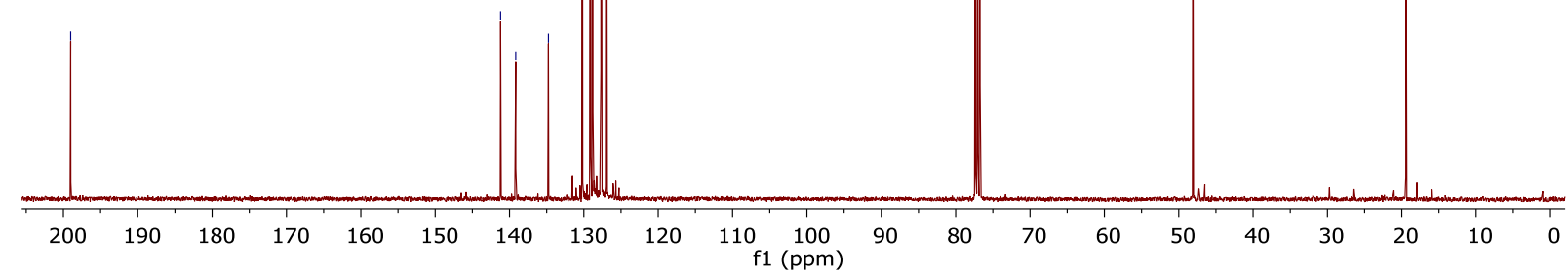


NMR-Spectra of 2-phenyl-1-(thiophen-2-yl)propan-1-one (3j)
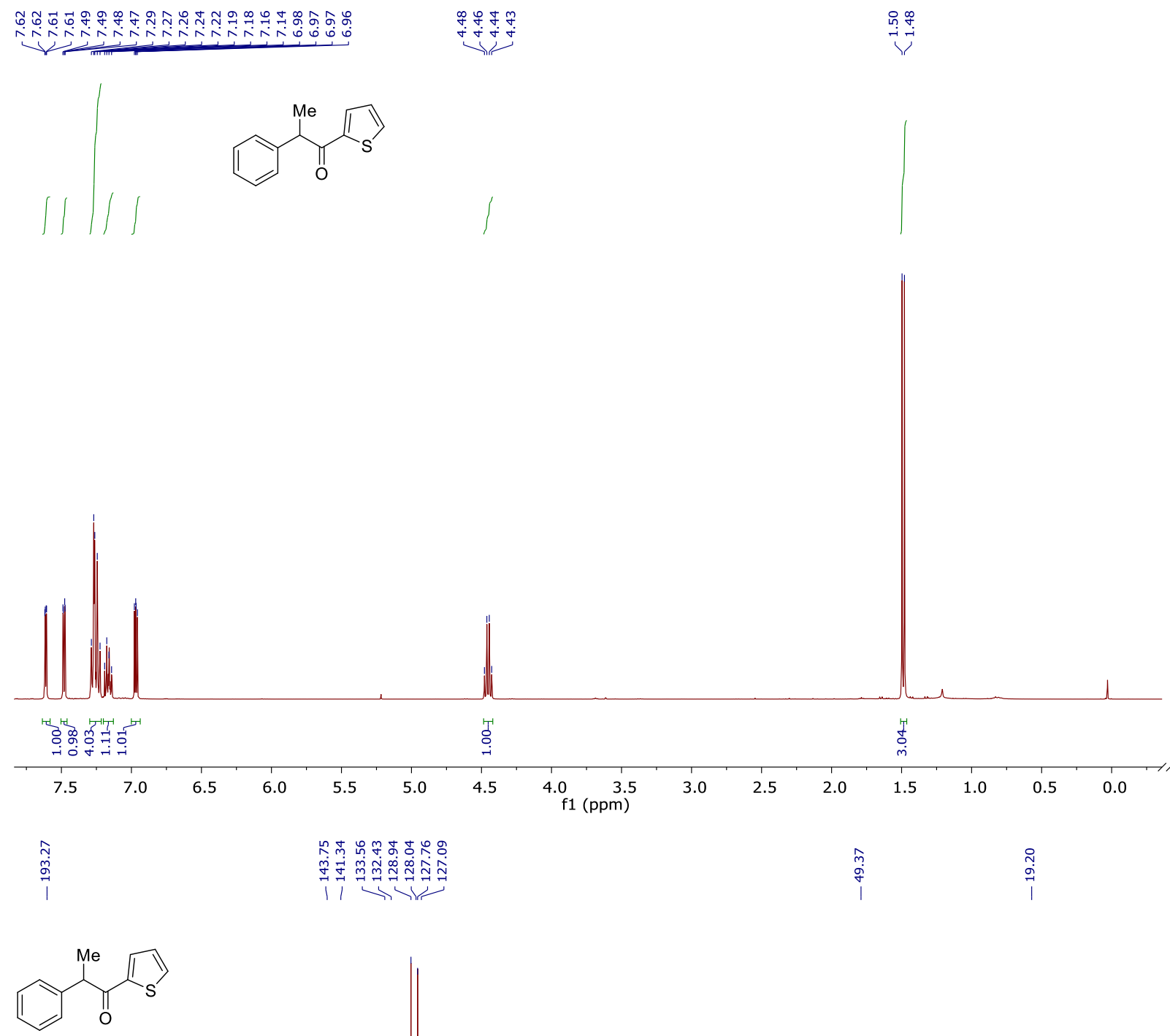

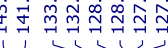

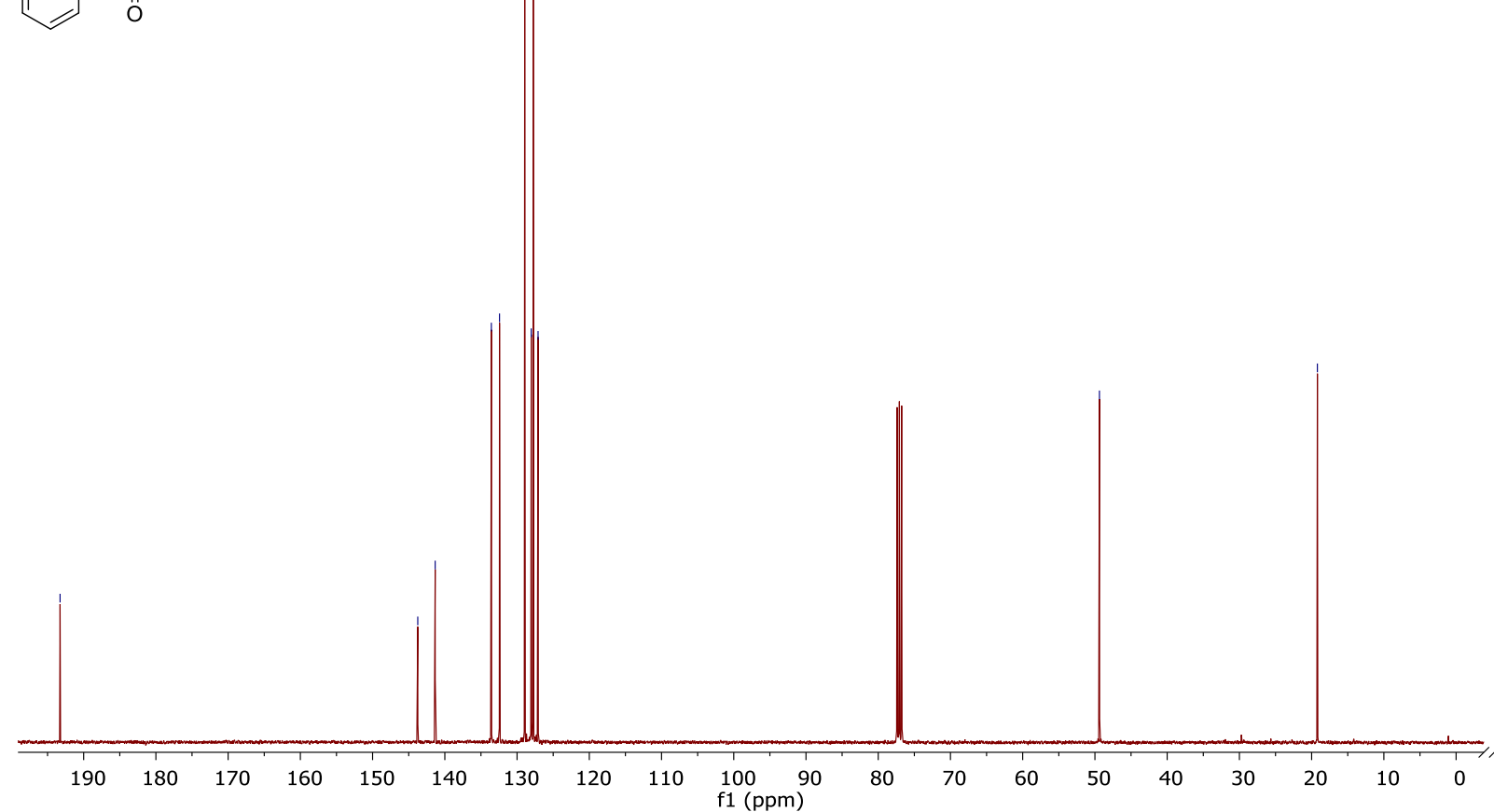


NMR-Spectra of 1-(4-methoxyphenyl)-2-phenylpropan-1-one (3k)
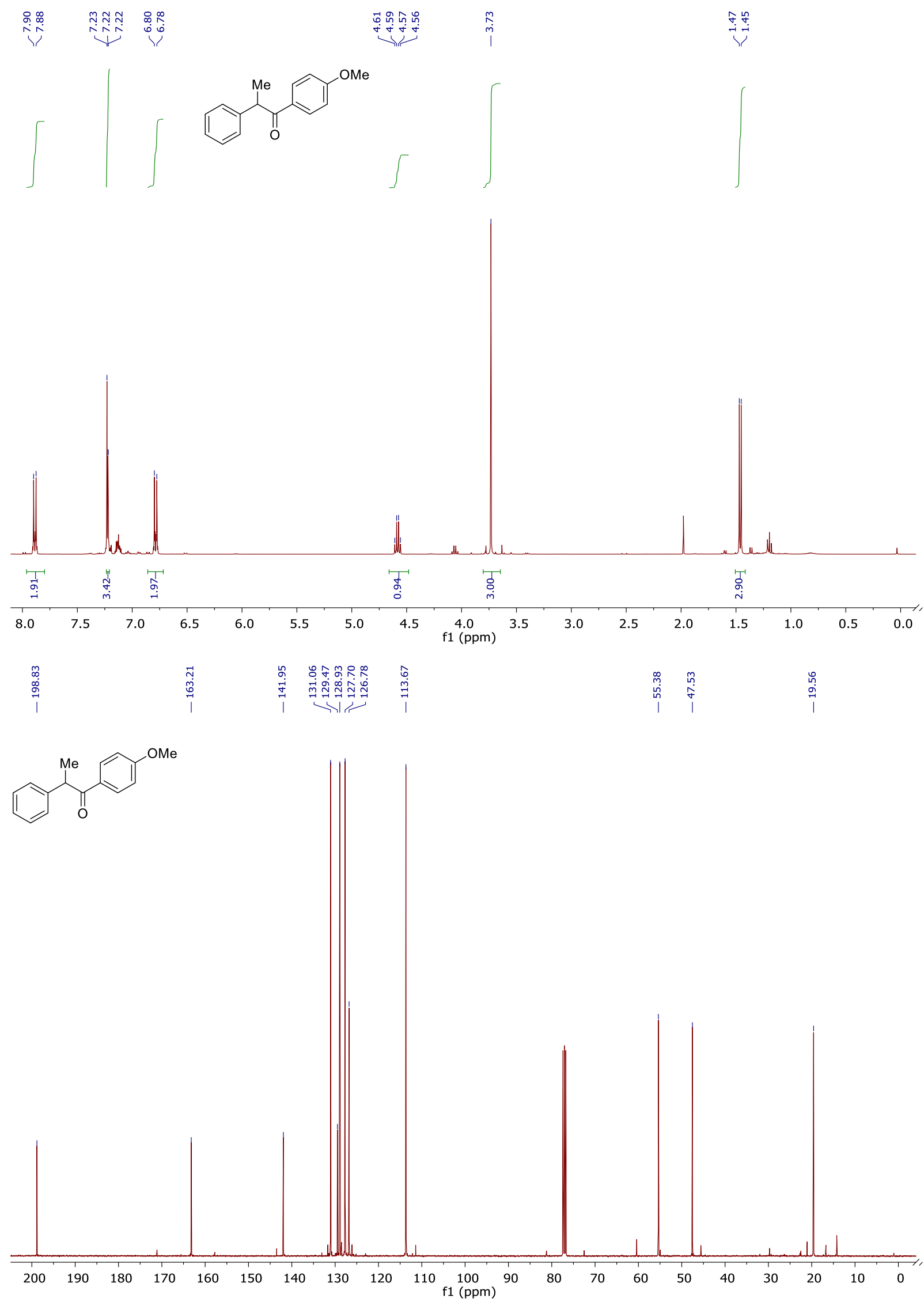
NMR-Spectra of ethyl 4-(4-chlorobenzoyl)benzoate (5a)

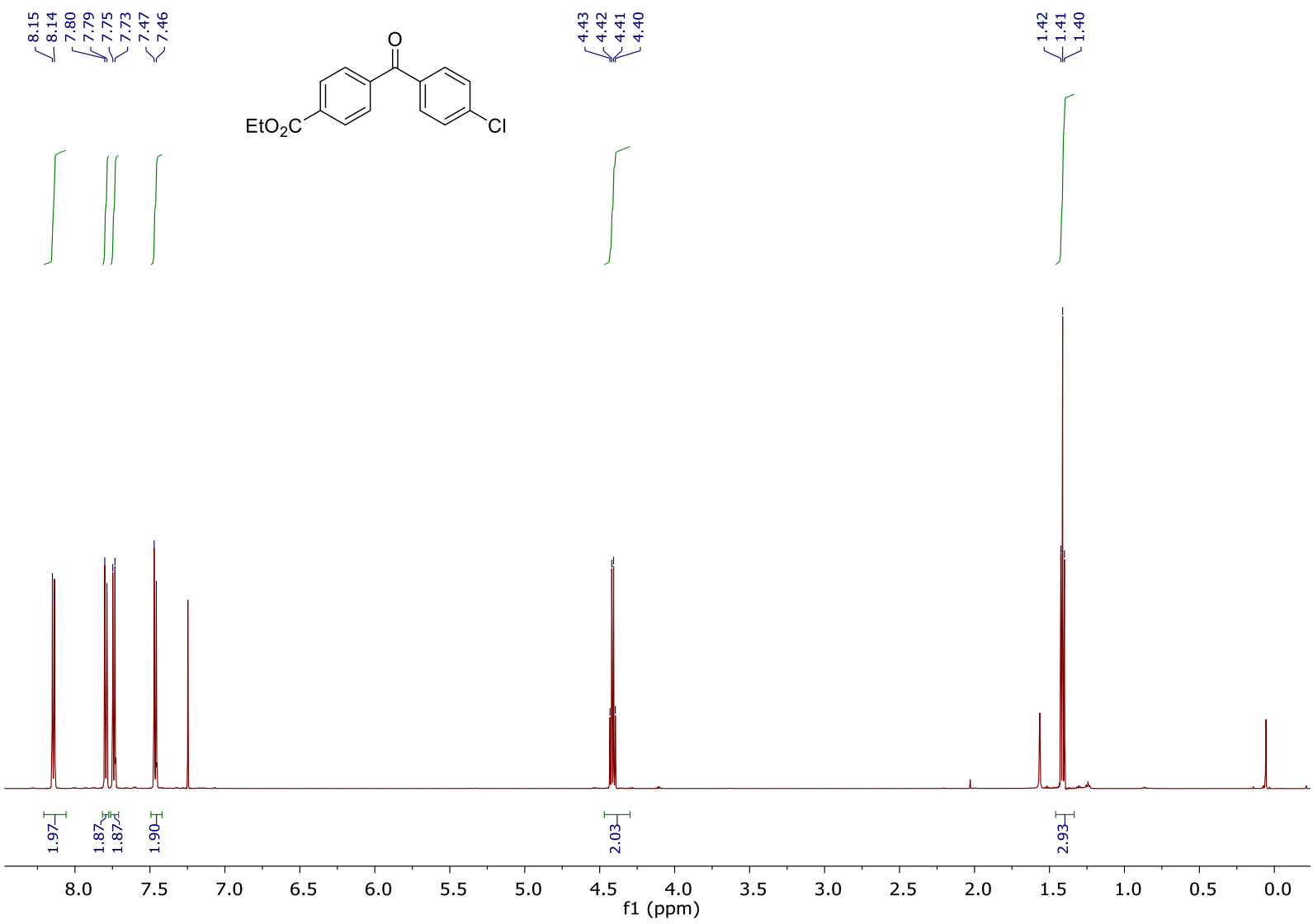

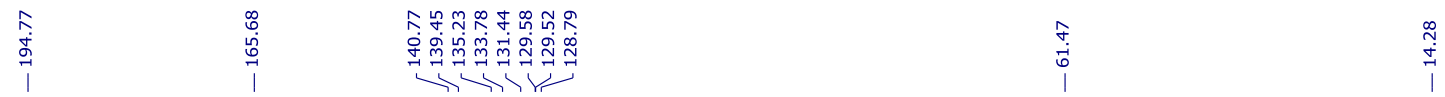
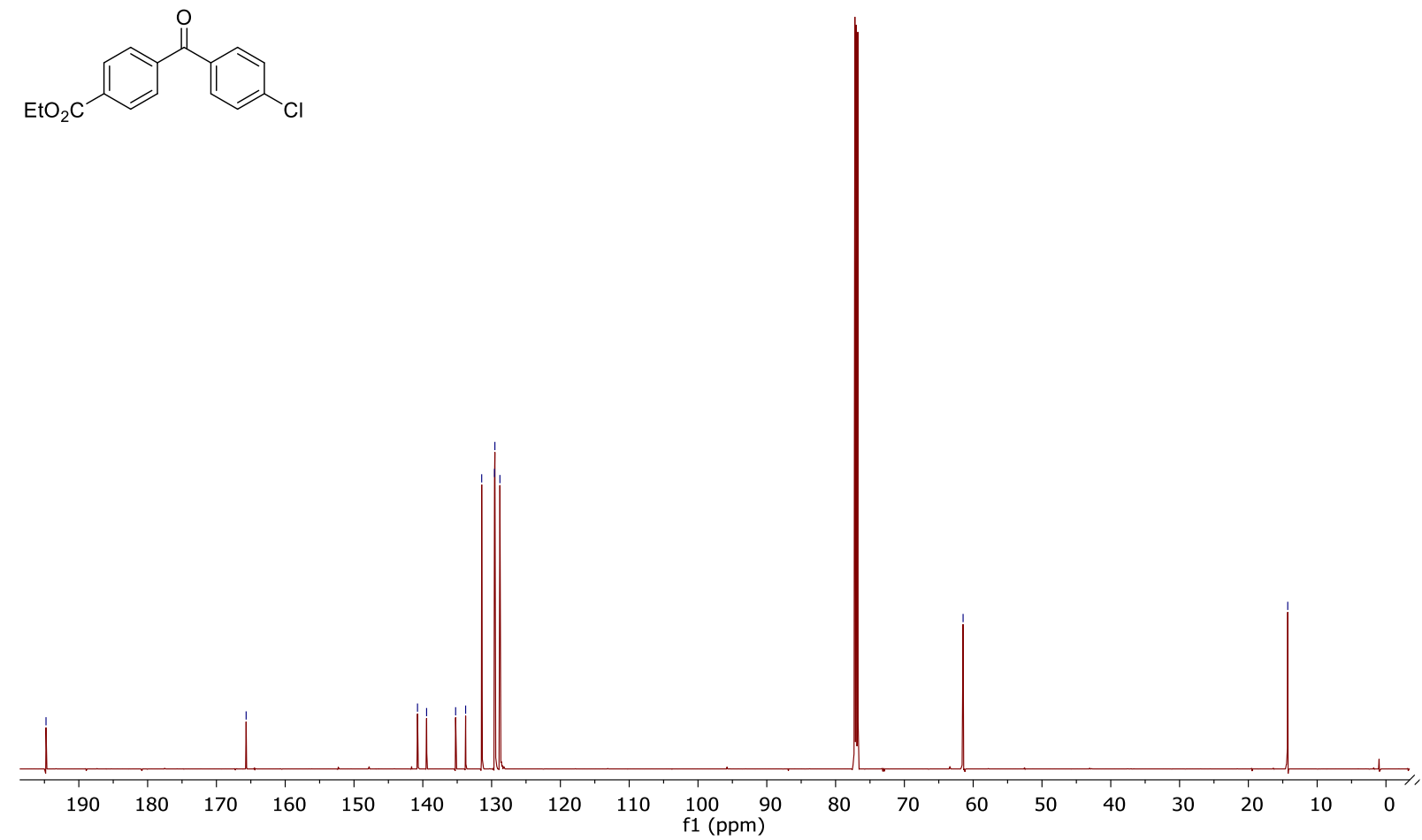
NMR-Spectra of ethyl 4-(4-(tert-butyl)benzoyl)benzoate (5b)

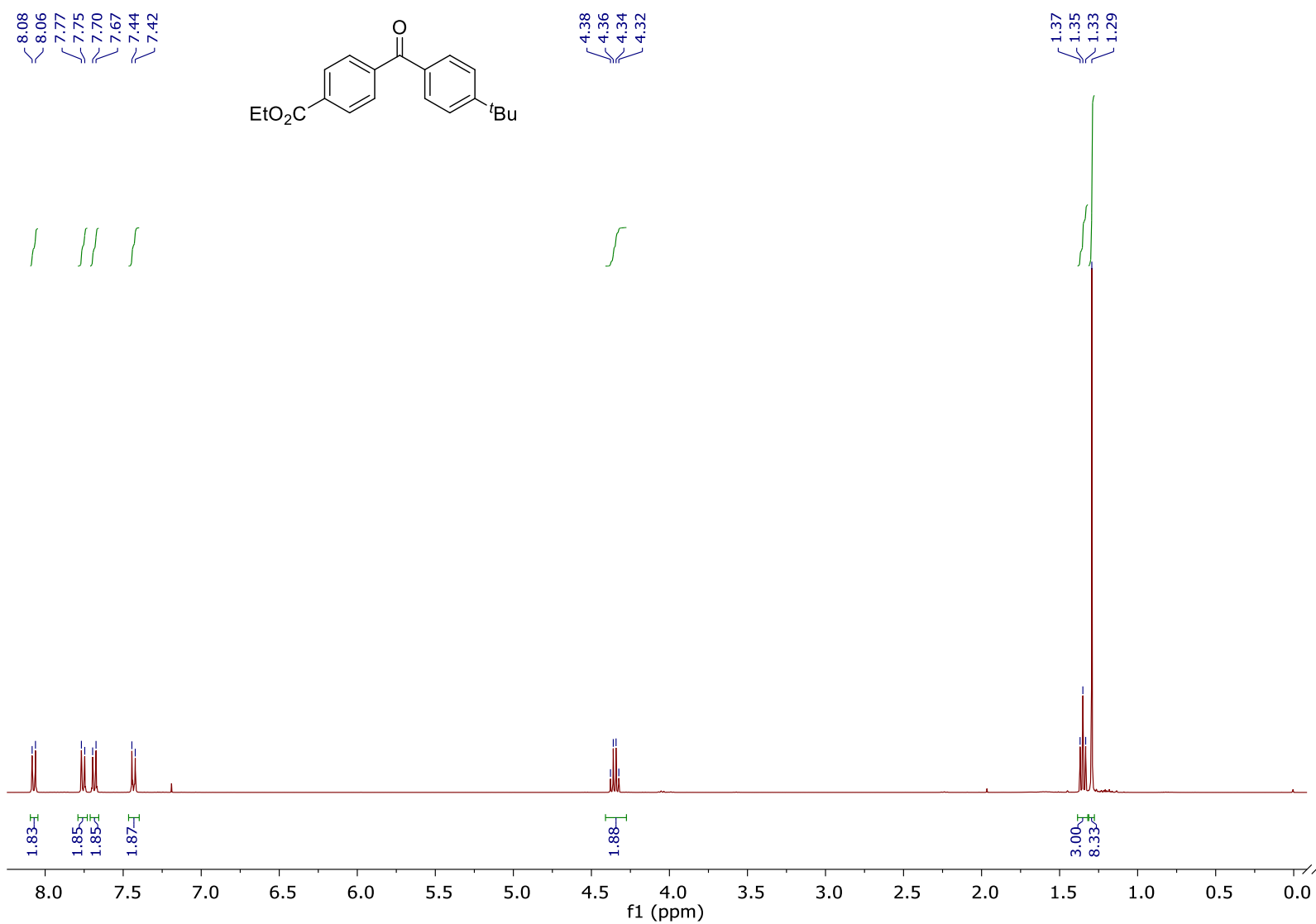

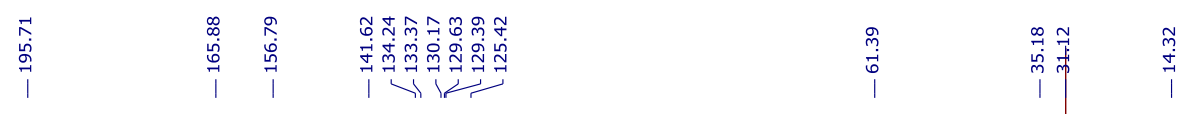<smiles>CCOC(=O)c1ccc(C(=O)c2ccc(Br)cc2)cc1</smiles>

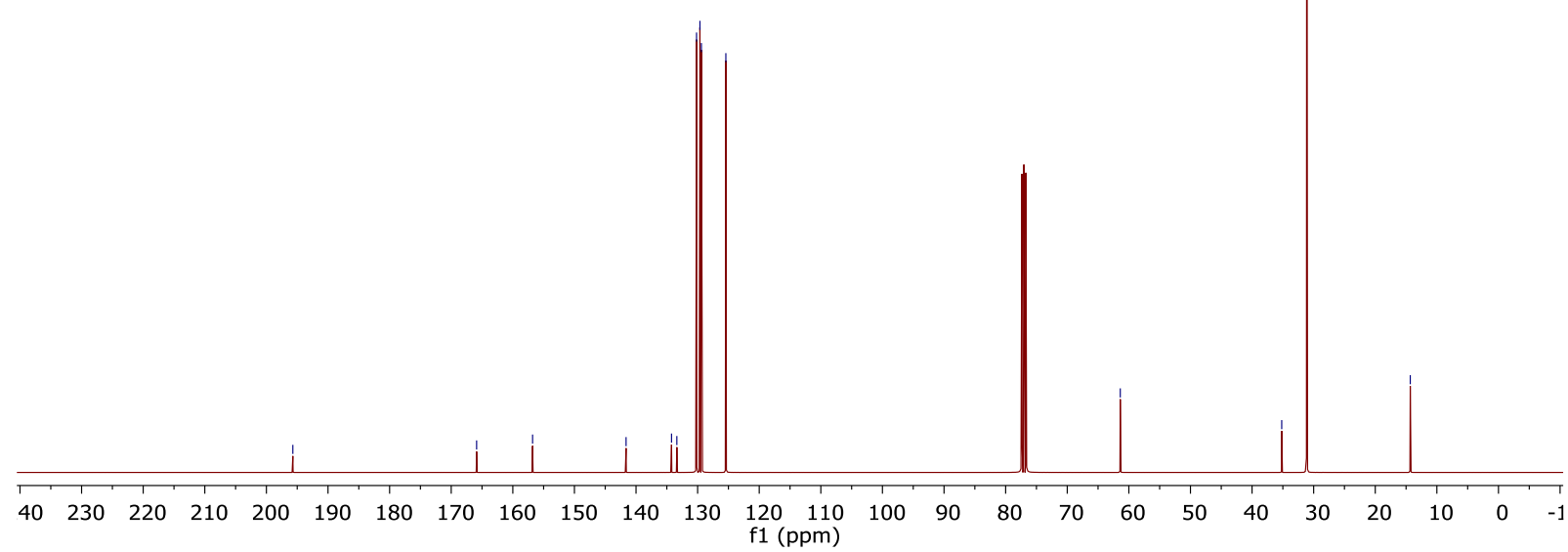


NMR-Spectra of ethyl 4-(4-fluorobenzoyl)benzoate (5c)

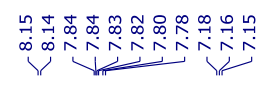
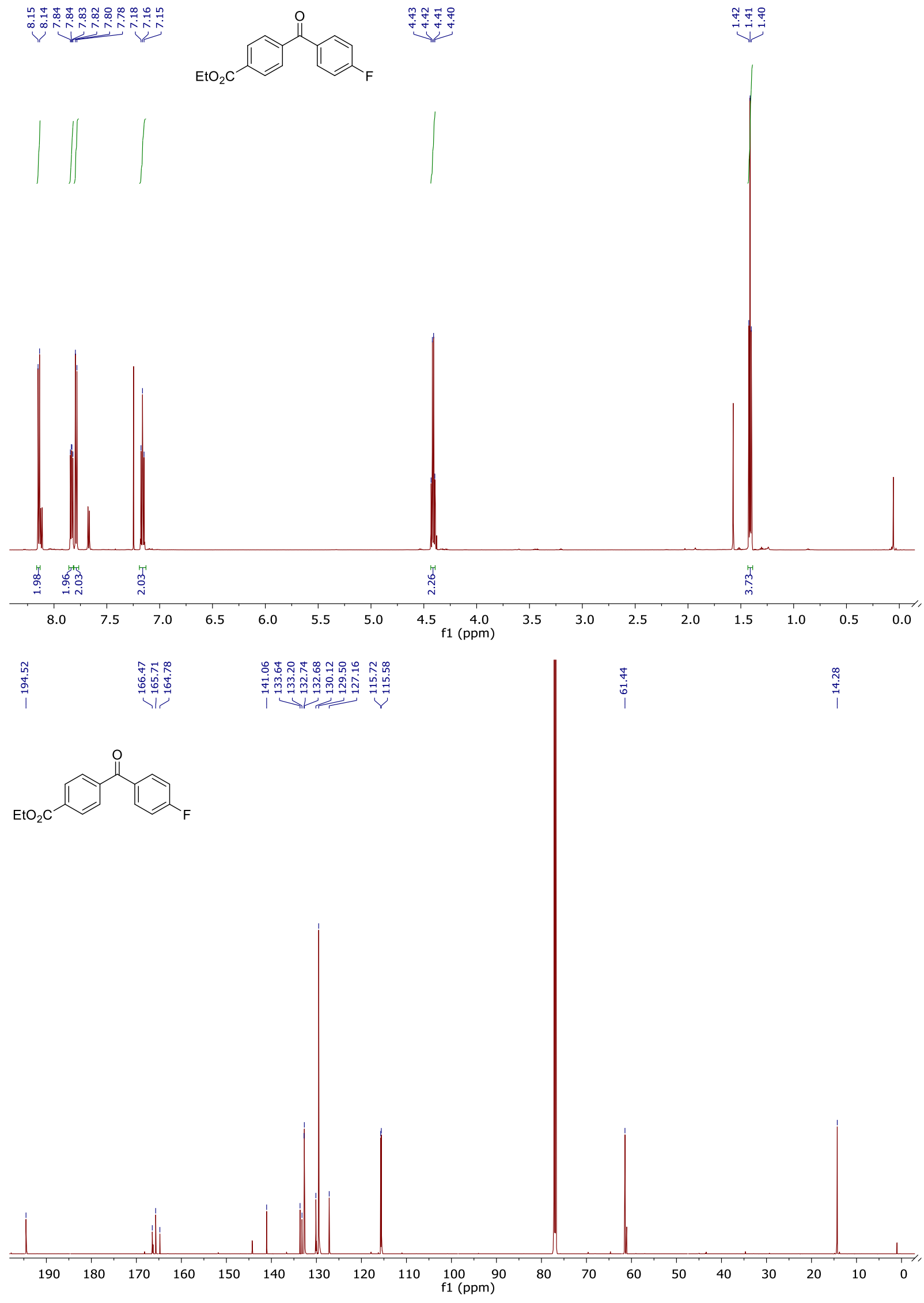

44 

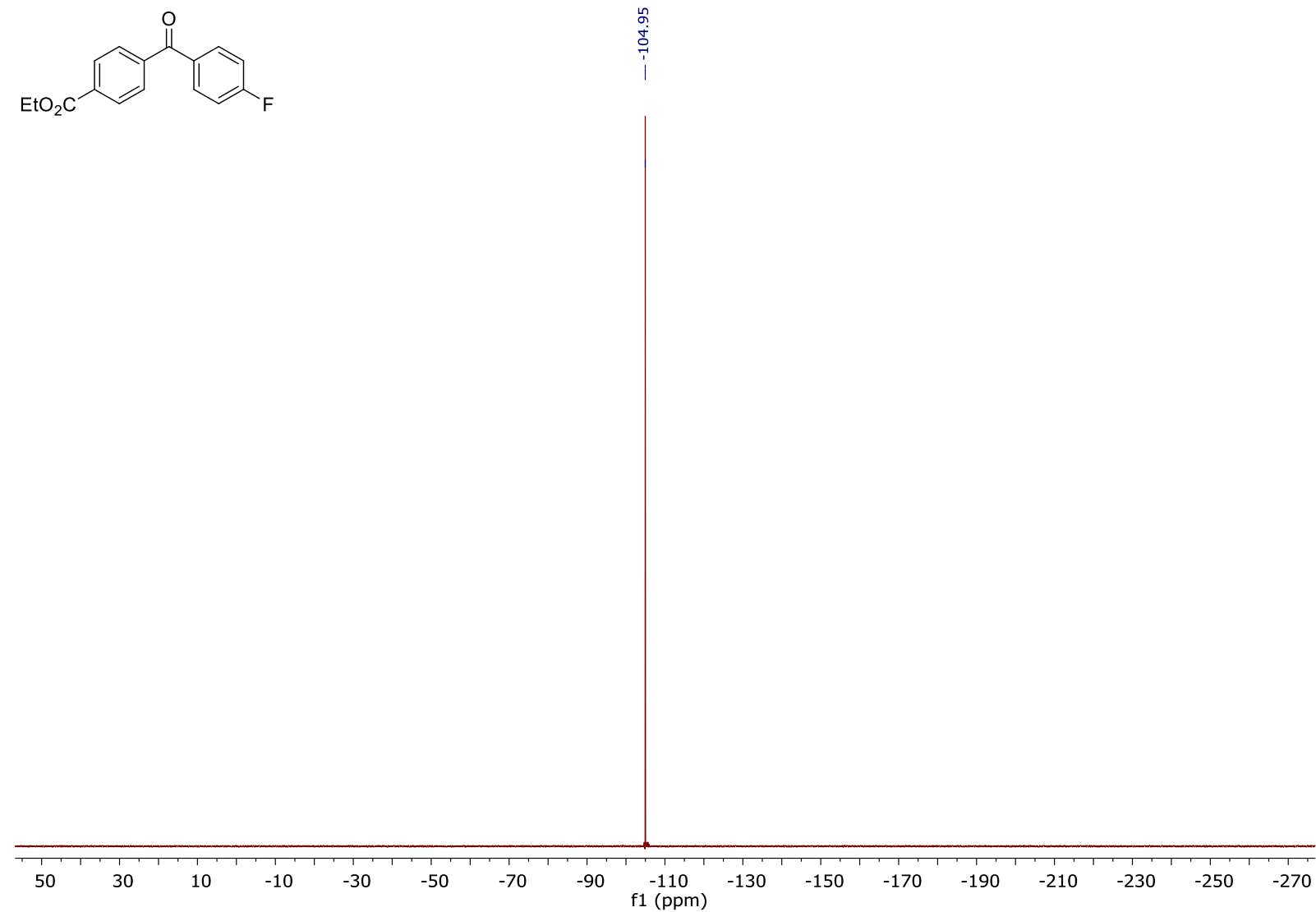

NMR-Spectra of ethyl 4-(4-bromobenzoyl)benzoate (5d)

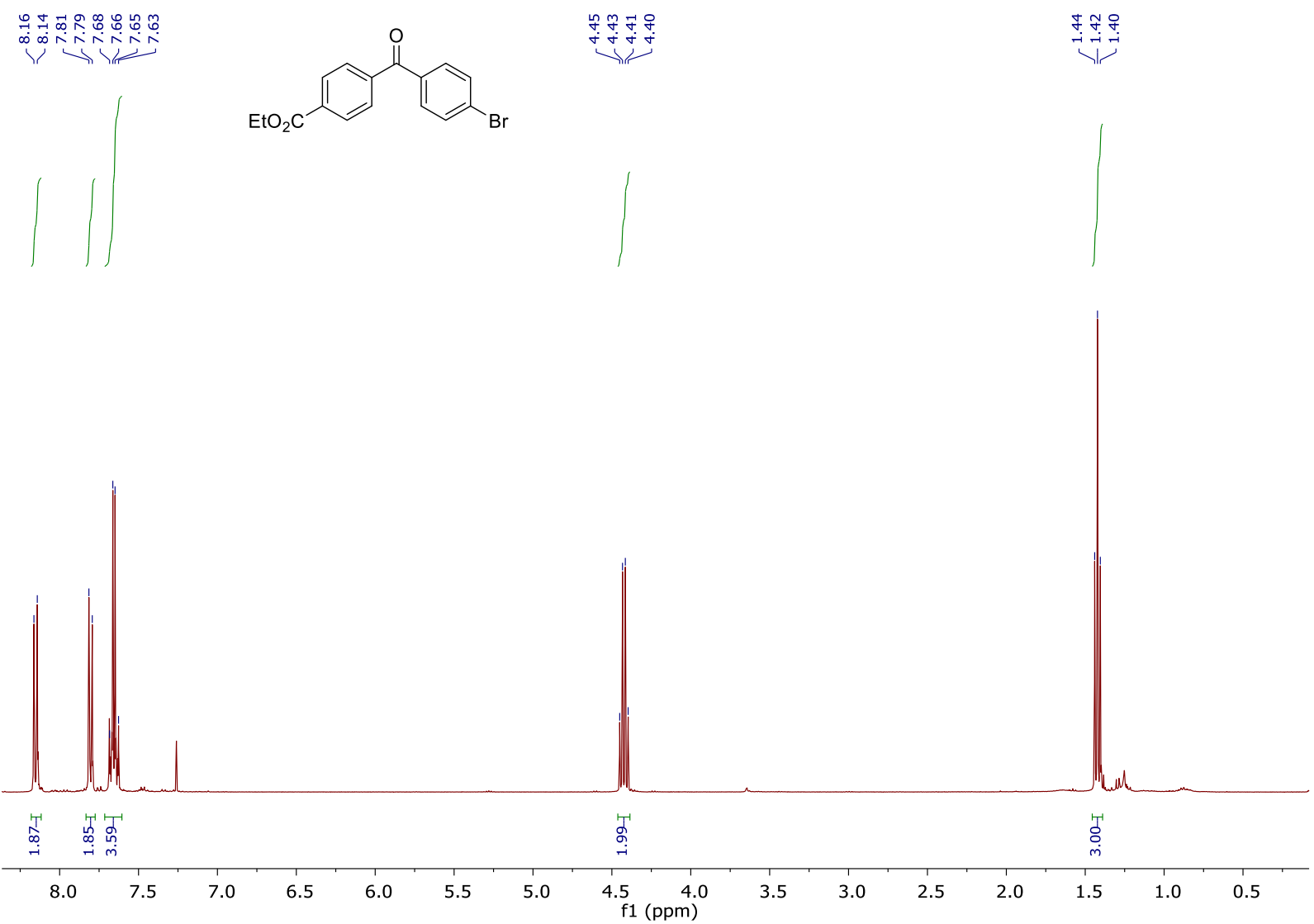




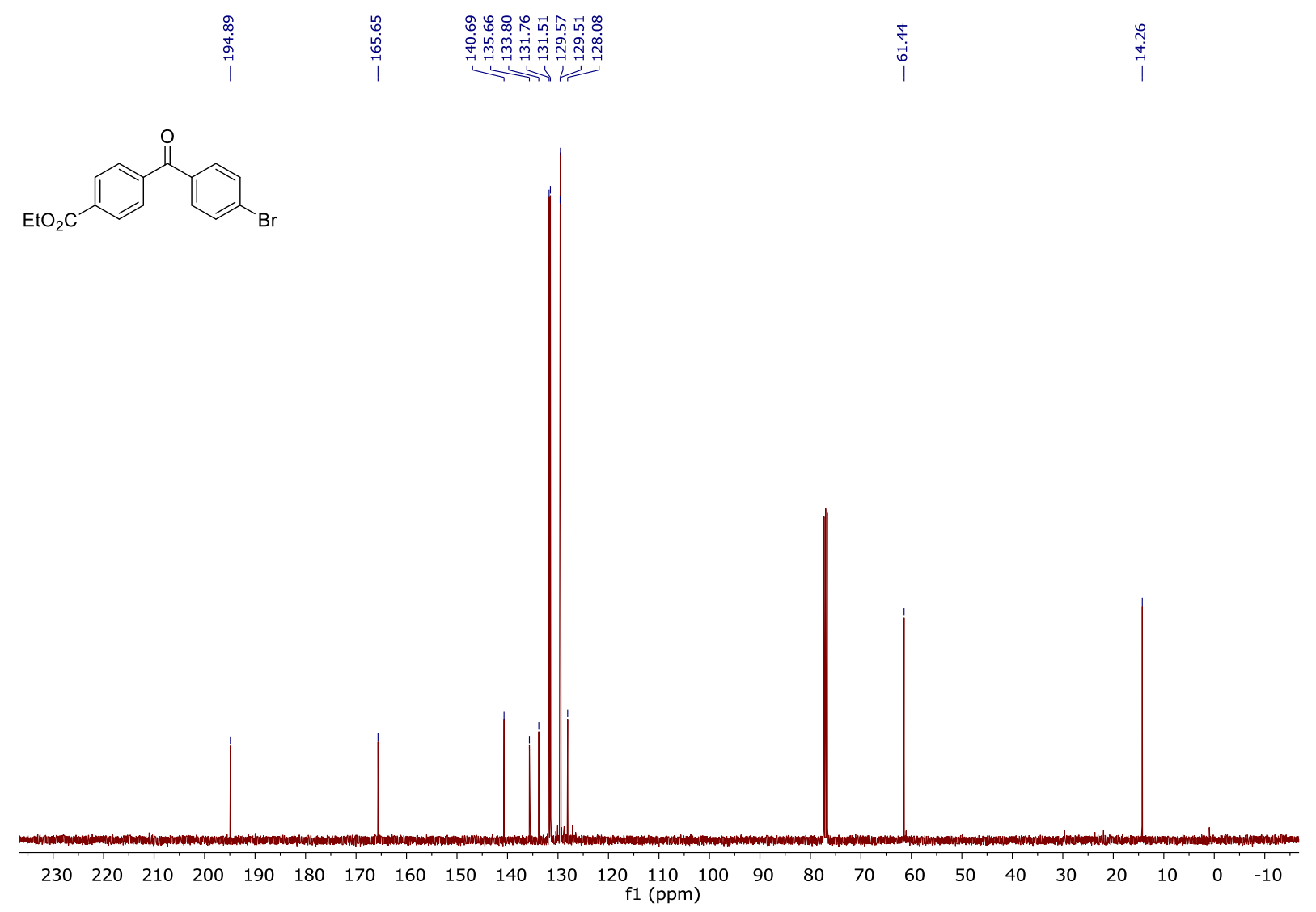

NMR-Spectra of ethyl 3-(4-chlorobenzoyl)benzoate (5e)

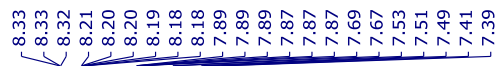

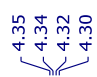

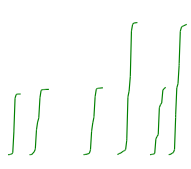<smiles>CCOC(=O)c1cccc(C(=O)c2ccc(Cl)cc2)c1</smiles>
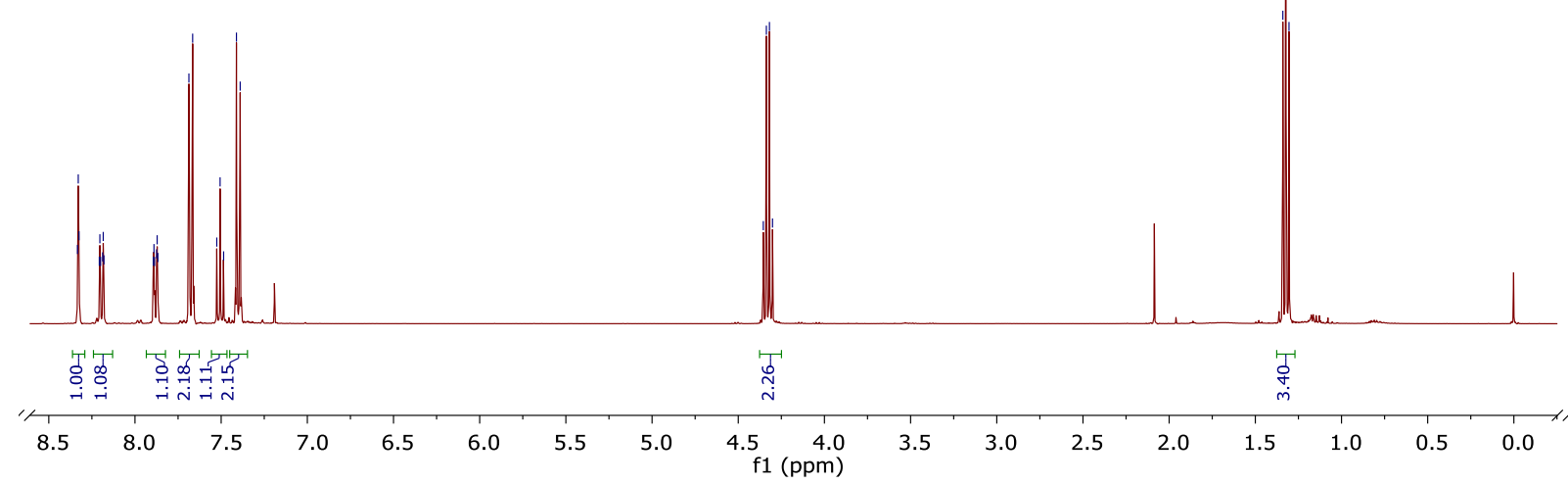


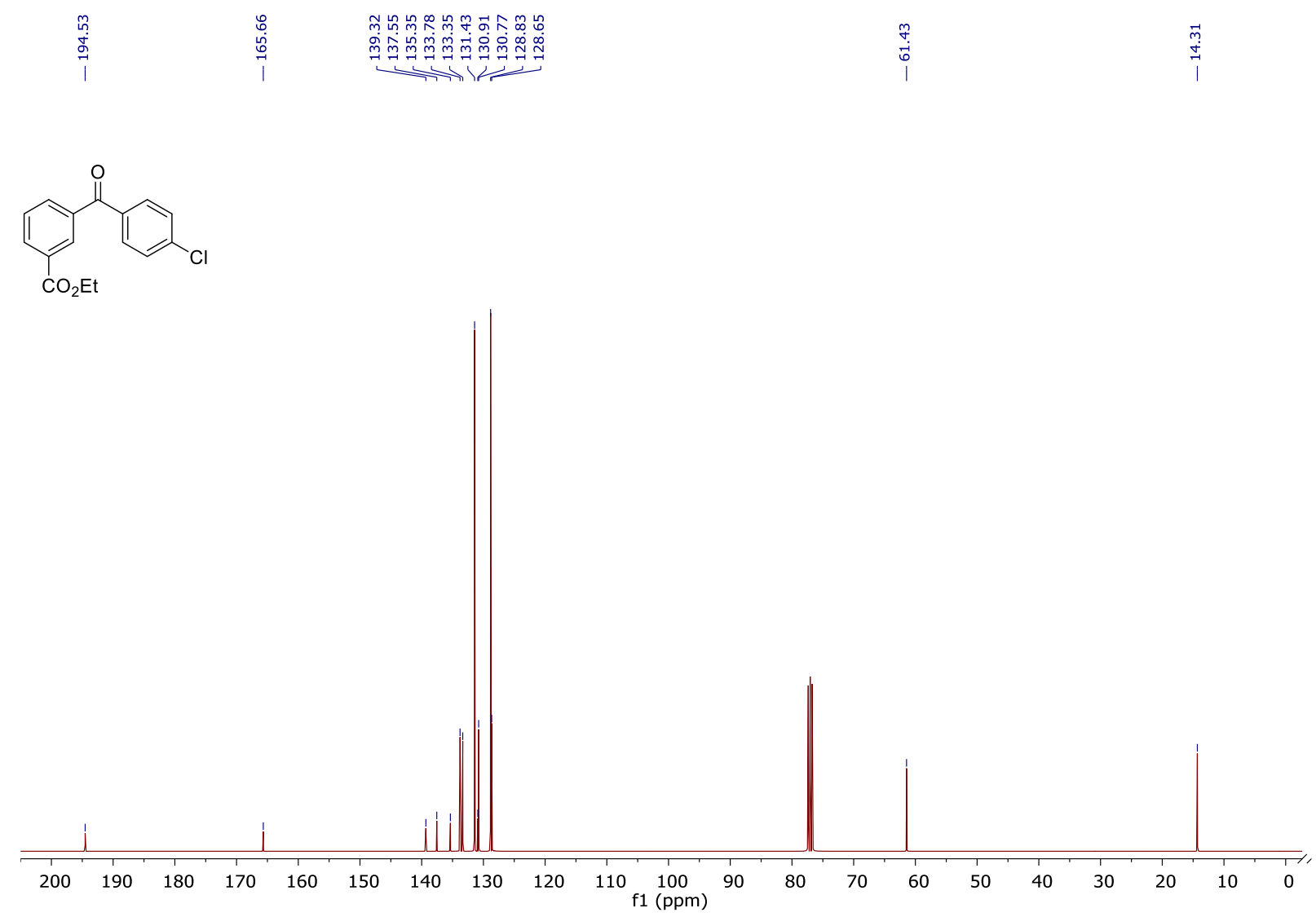

NMR-Spectra of ethyl 3-(4-(tert-butyl)benzoyl)benzoate (5f)

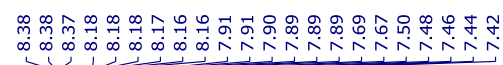

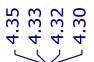

$51 / 1$<smiles>CCOC(=O)c1cccc(C(=O)c2ccc(Br)cc2)c1</smiles>
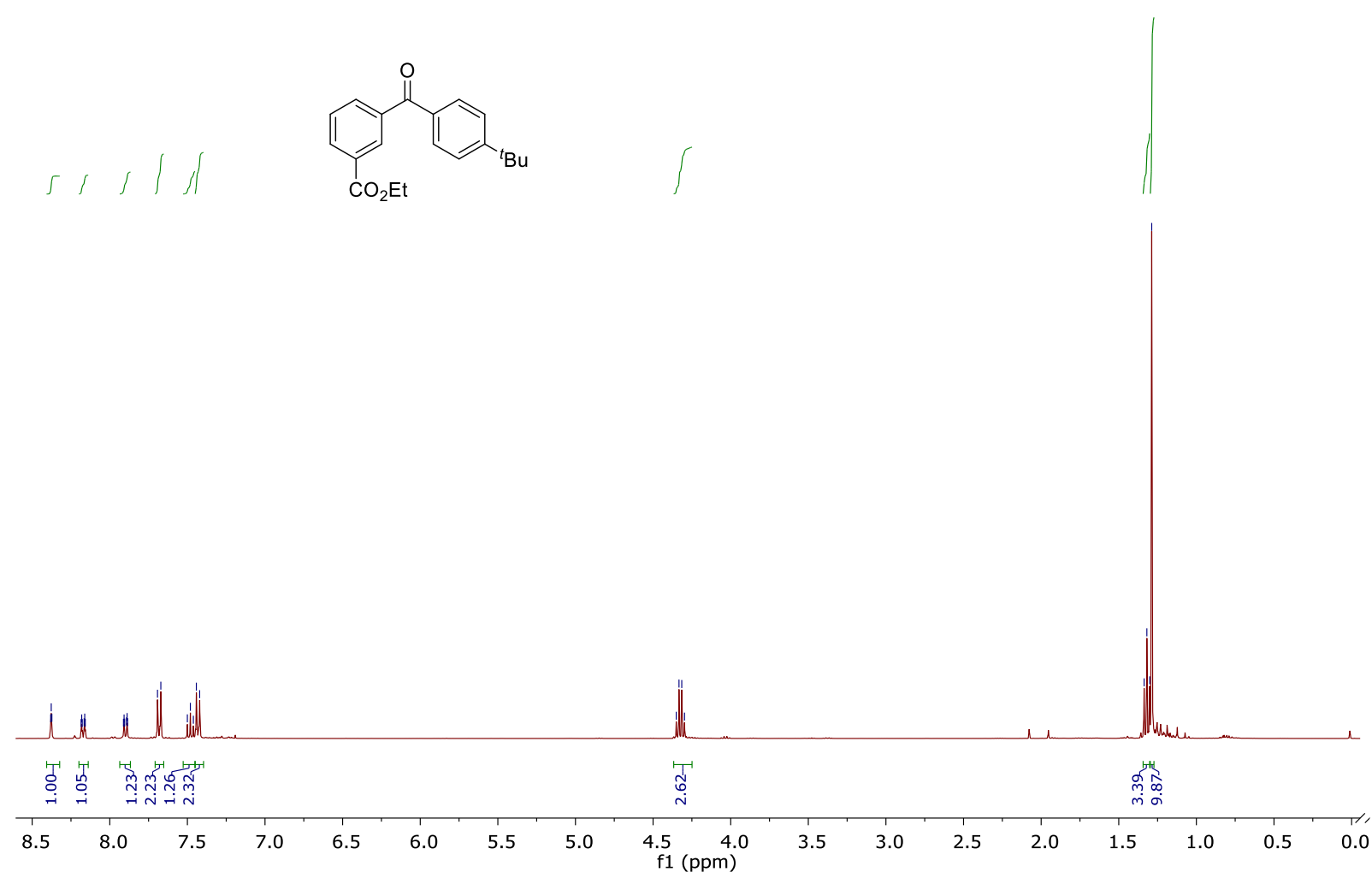


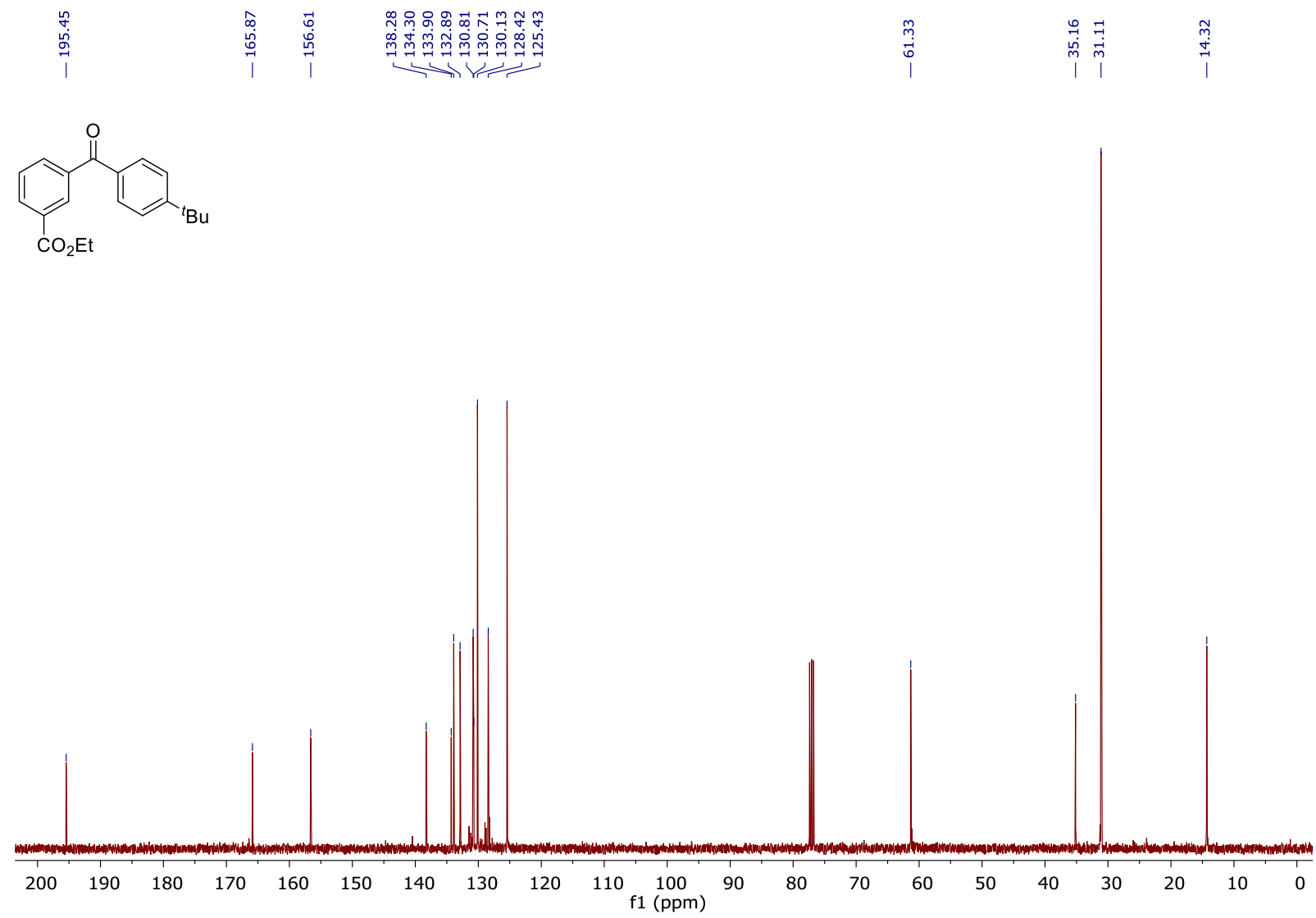

NMR-Spectra of ethyl 4-(cyclohexanecarbonyl)benzoate (5g)

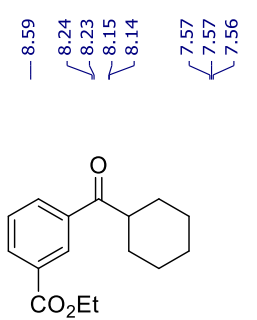

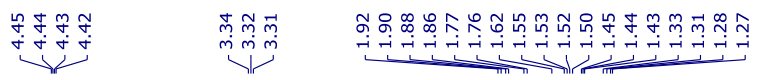
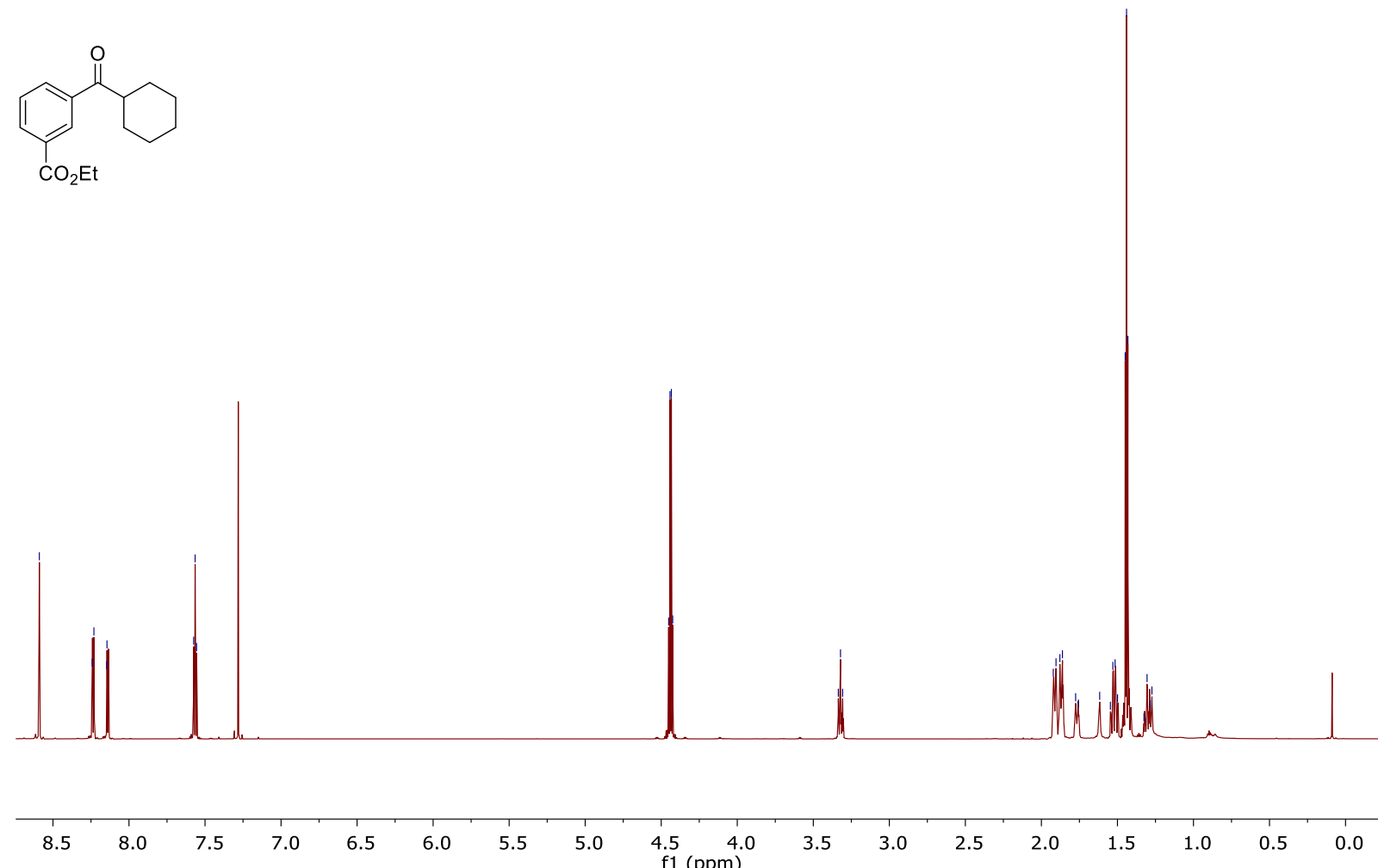

$\begin{array}{lllllllll}8.5 & 8.0 & 7.5 & 7.0 & 6.5 & 6.0 & 5.5 & 5.0 & 4.5 \\ \mathrm{f} 1(\mathrm{ppm})\end{array}$ 


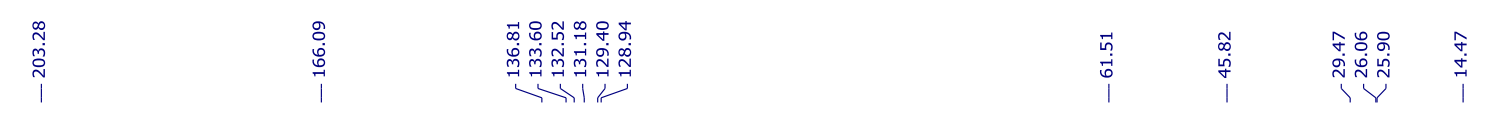<smiles>CCOc1cccc(C(=O)C2CCCCC2)c1</smiles>

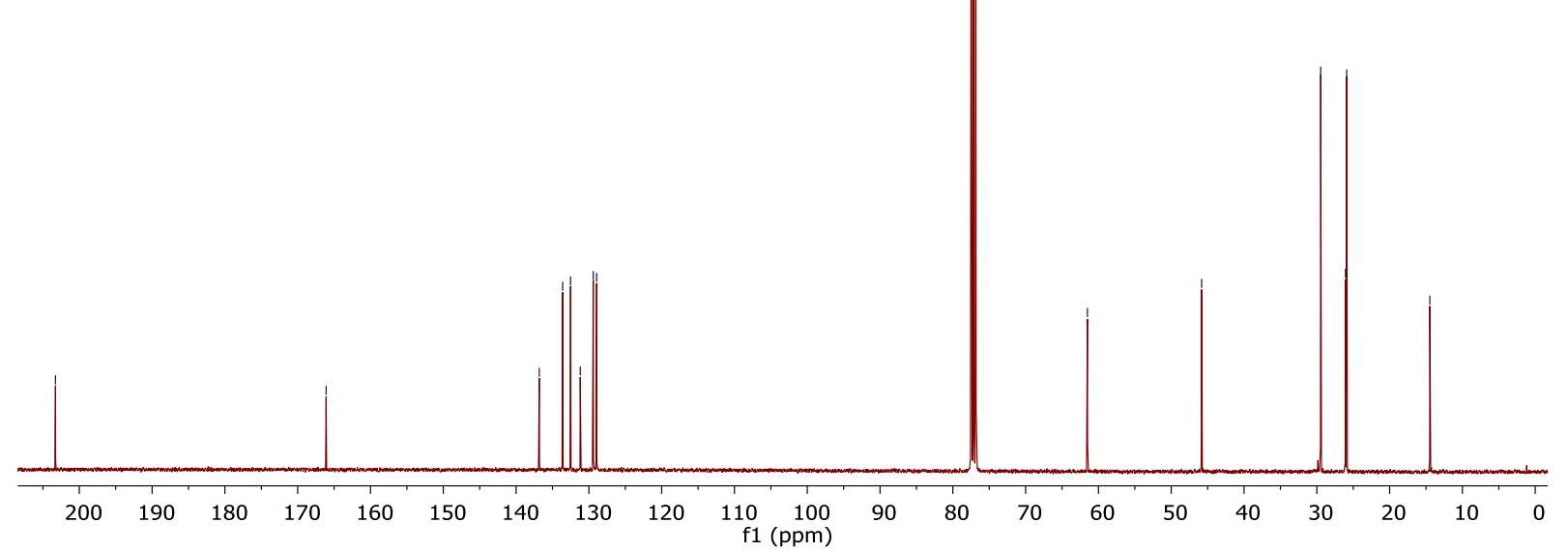

NMR-Spectra of ethyl 2-(4-chlorobenzoyl)benzoate (5h)
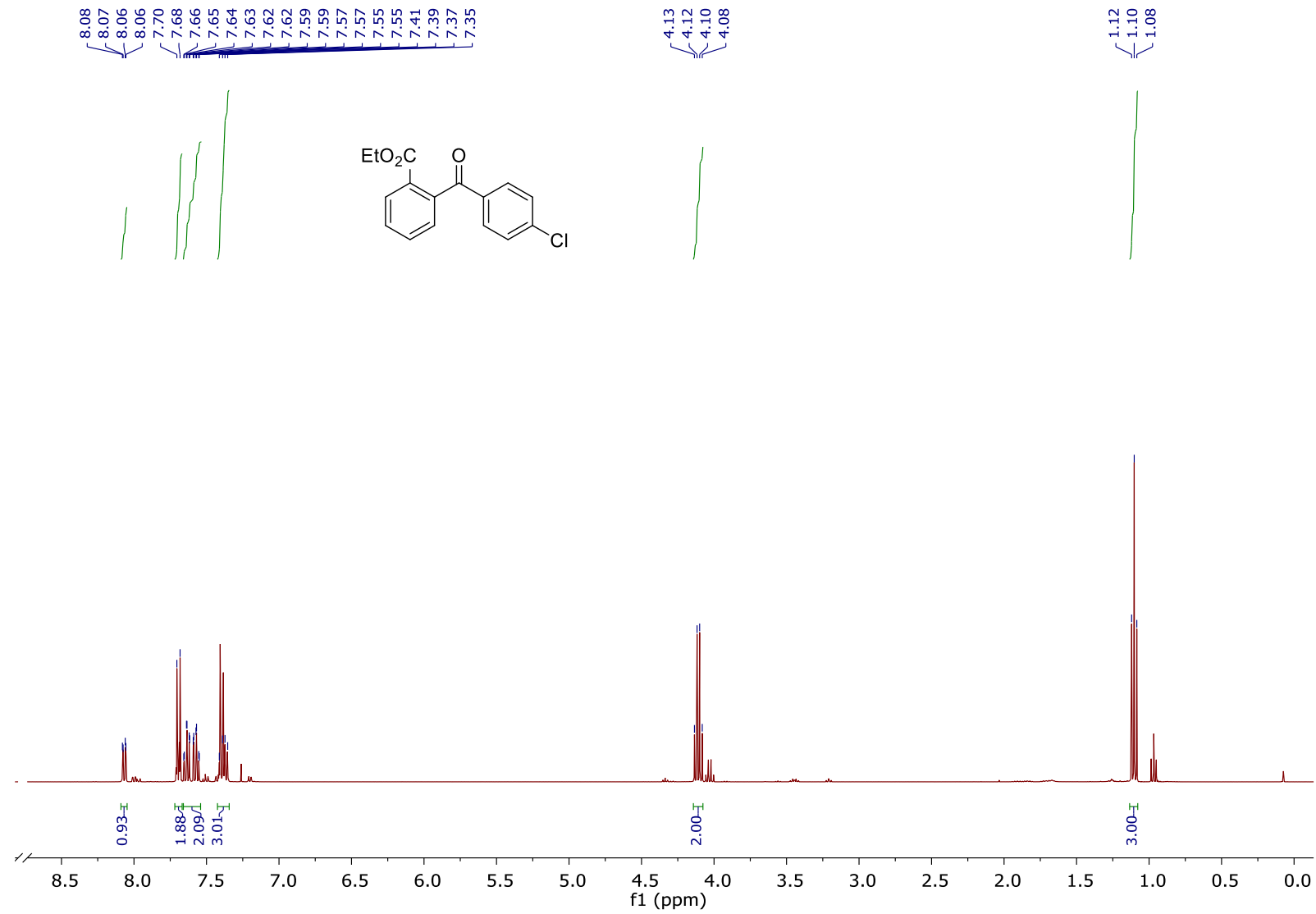


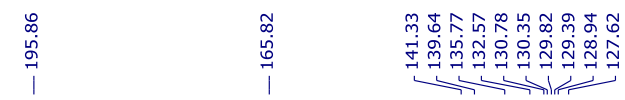

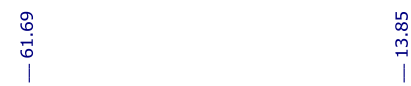<smiles>CCOc1ccccc1C(=O)c1ccc(Cl)cc1</smiles>

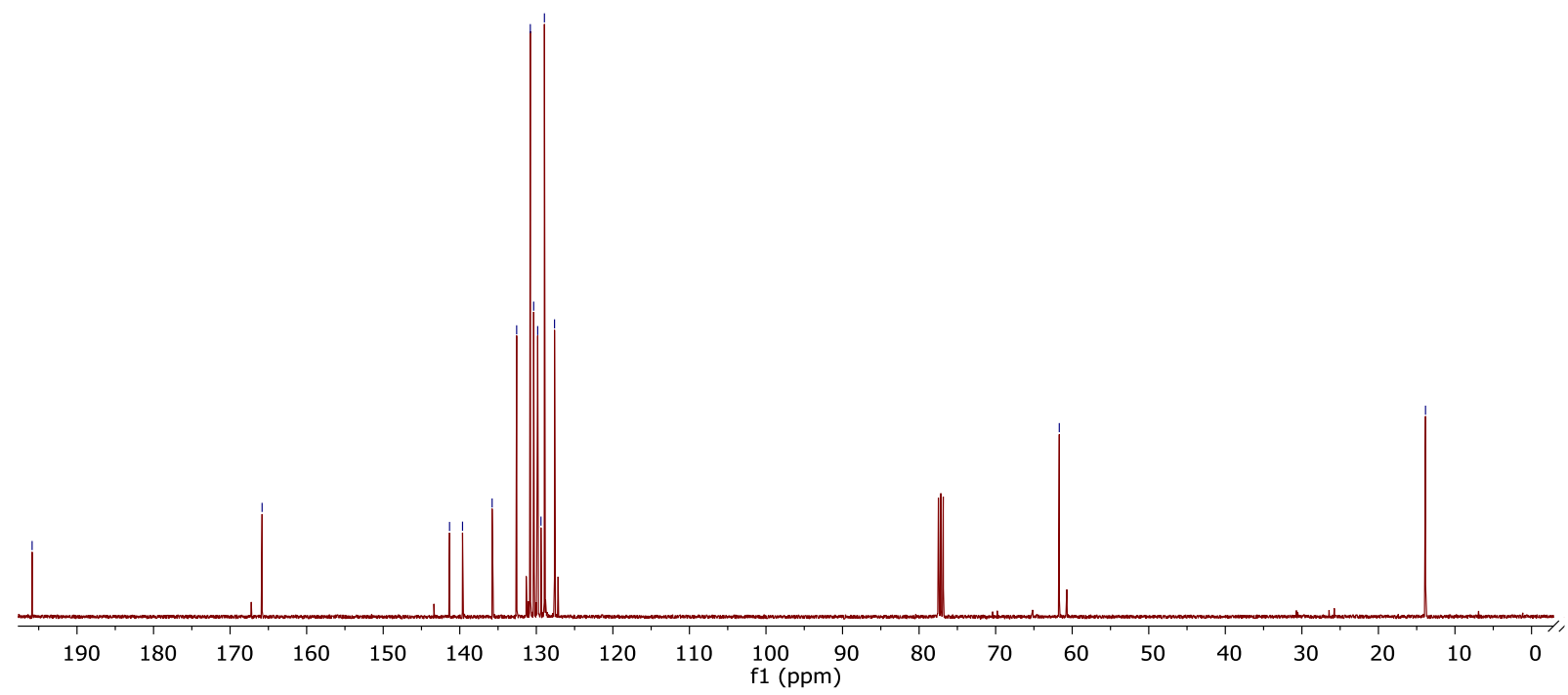

NMR-Spectra of ethyl 2-(4-cyanobenzoyl)benzoate (5i)
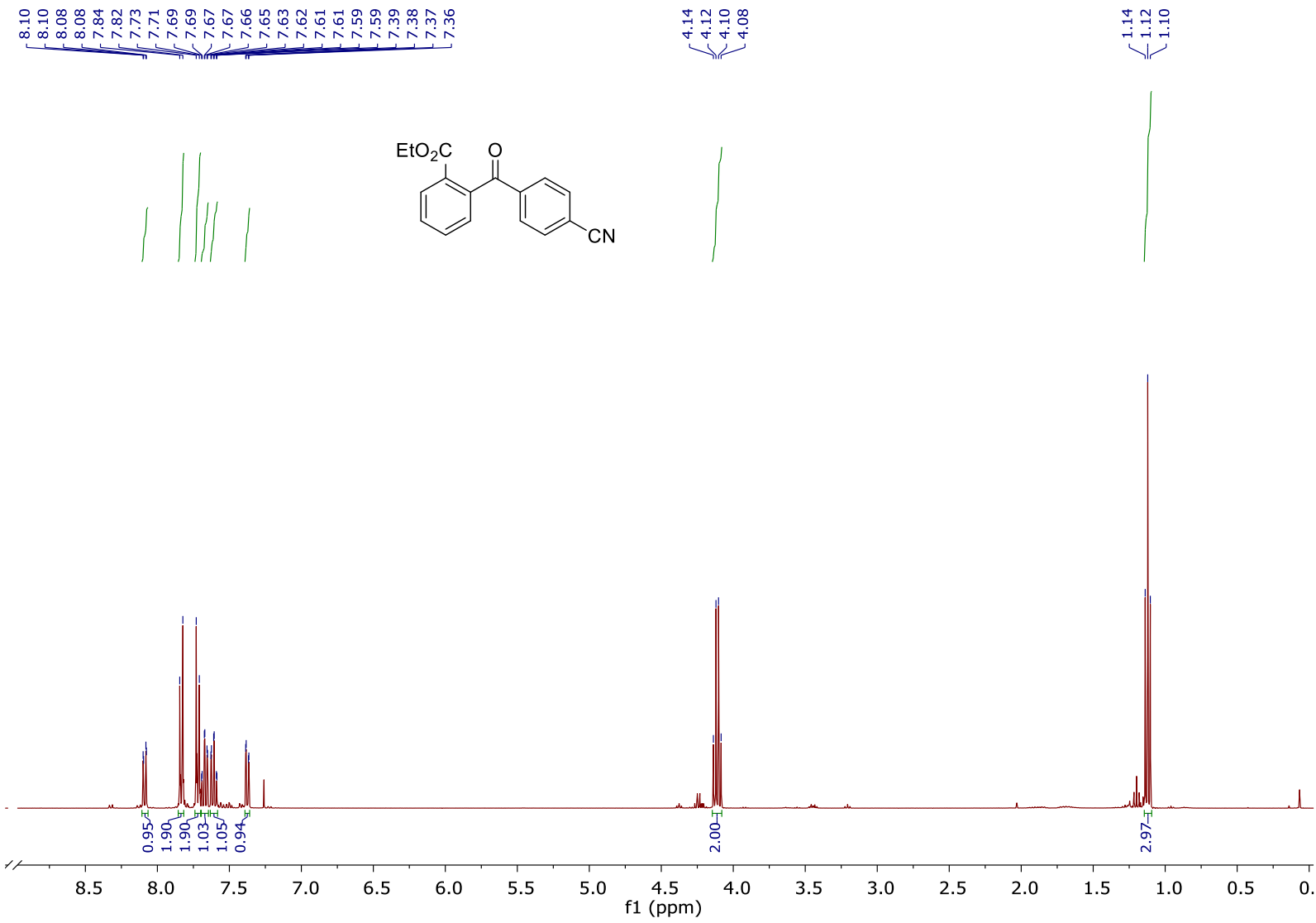


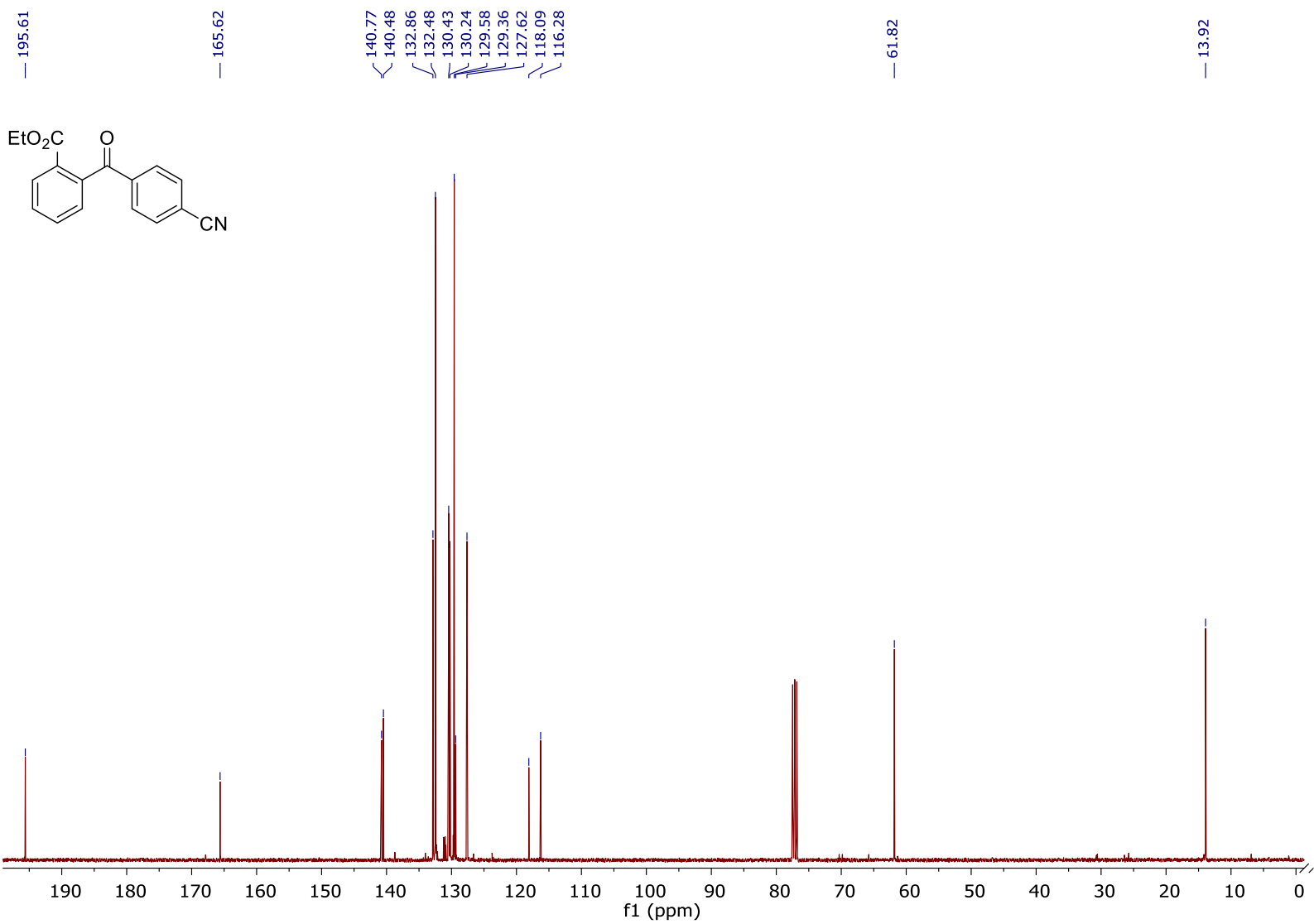

NMR-Spectra of (4-(tert-butyl)phenyl)(4-(trifluoromethyl)phenyl)methanone (5j)

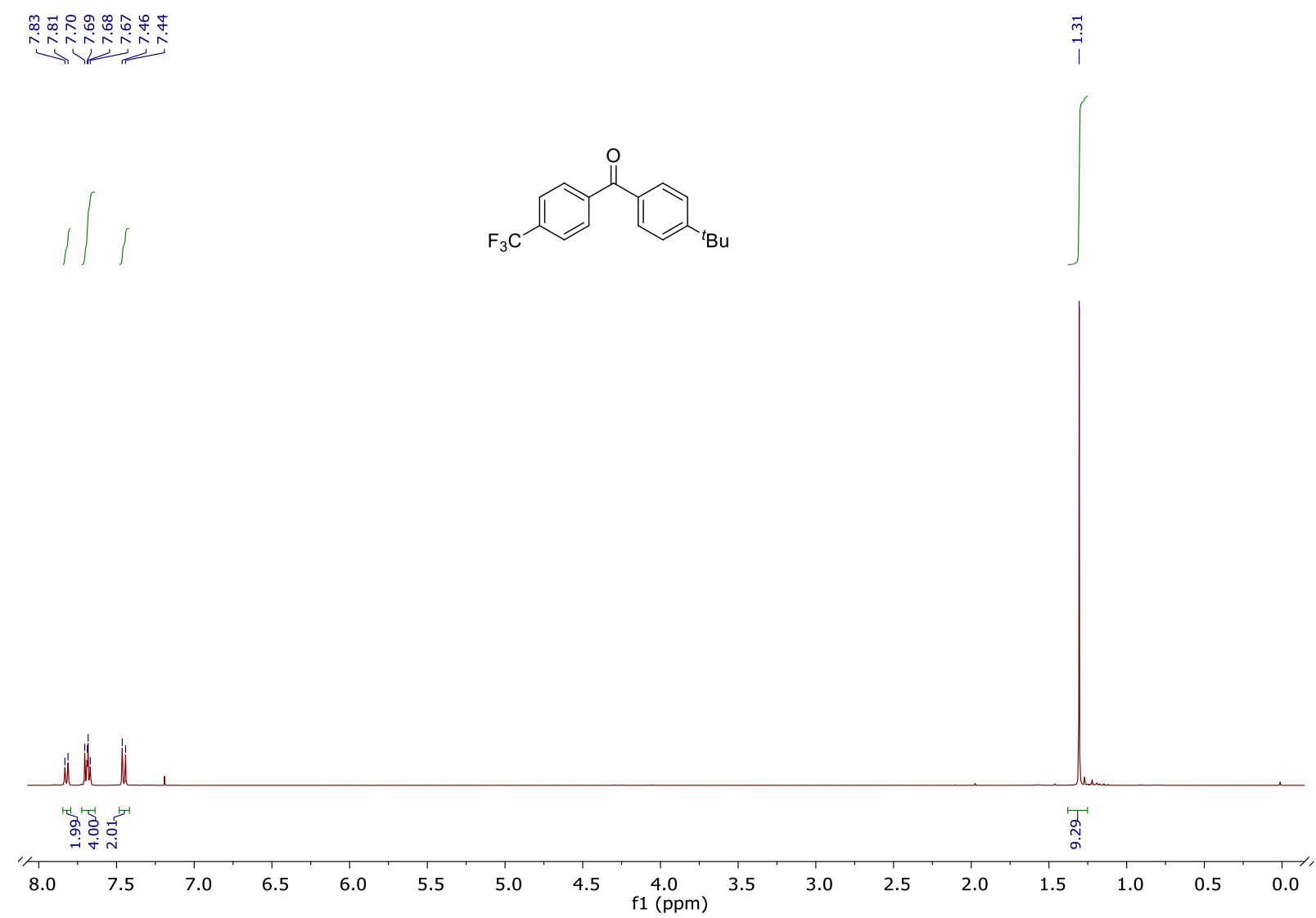




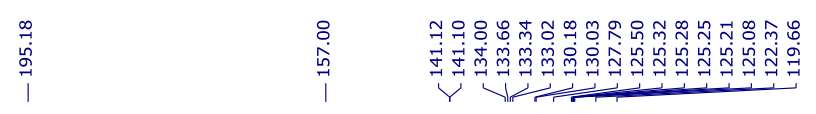

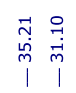
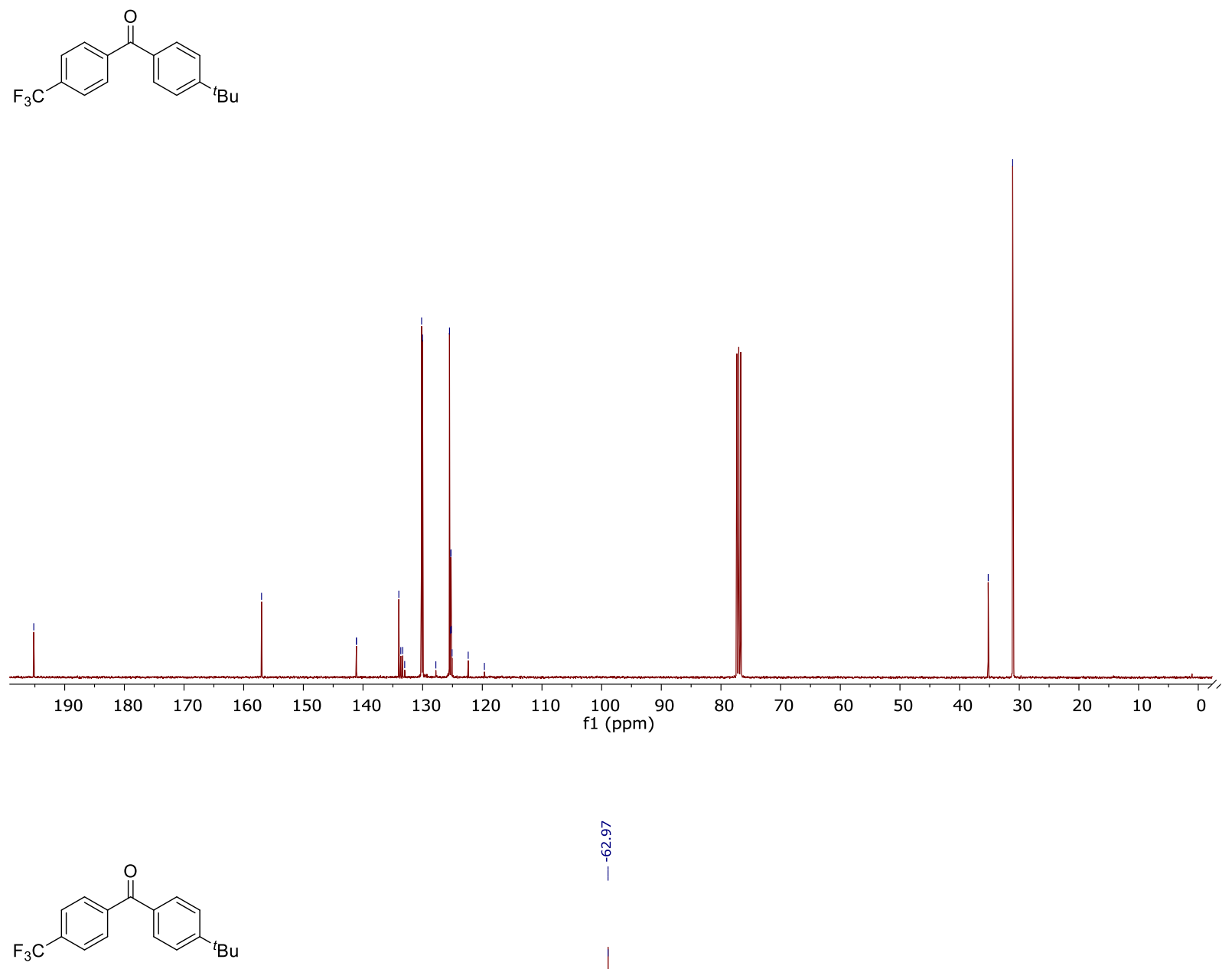

ลิ̀

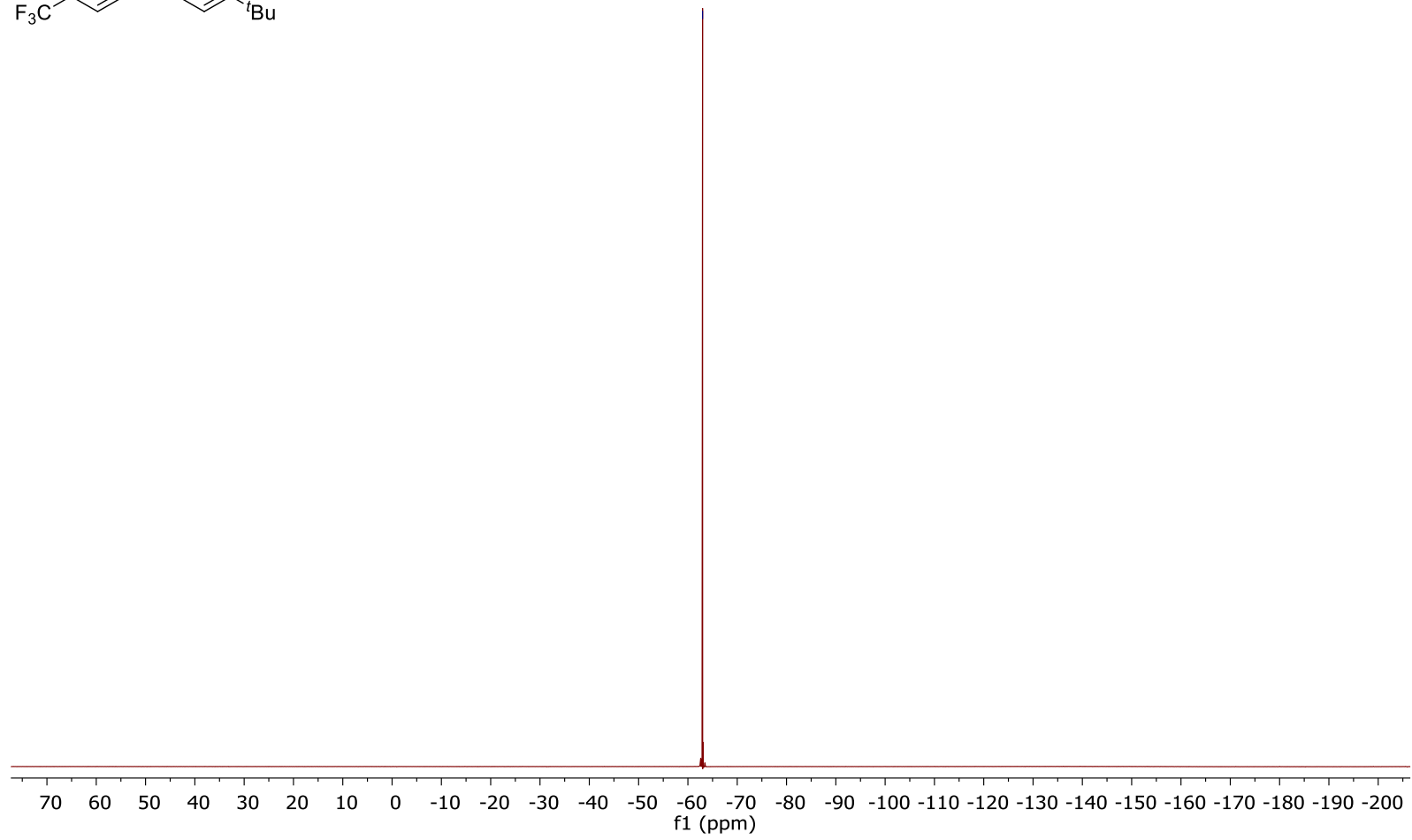

52 
NMR-Spectra of (4-methoxyphenyl)(4-(trifluoromethyl)phenyl)methanone (5k)
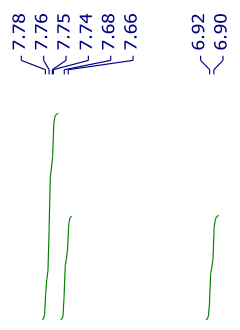

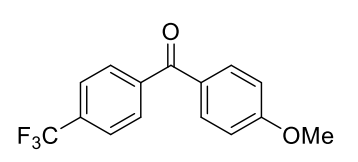

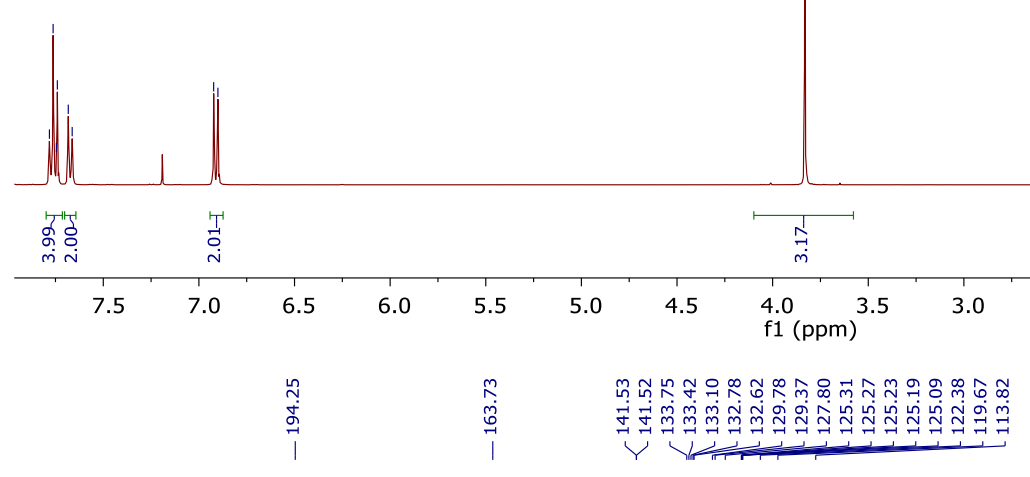
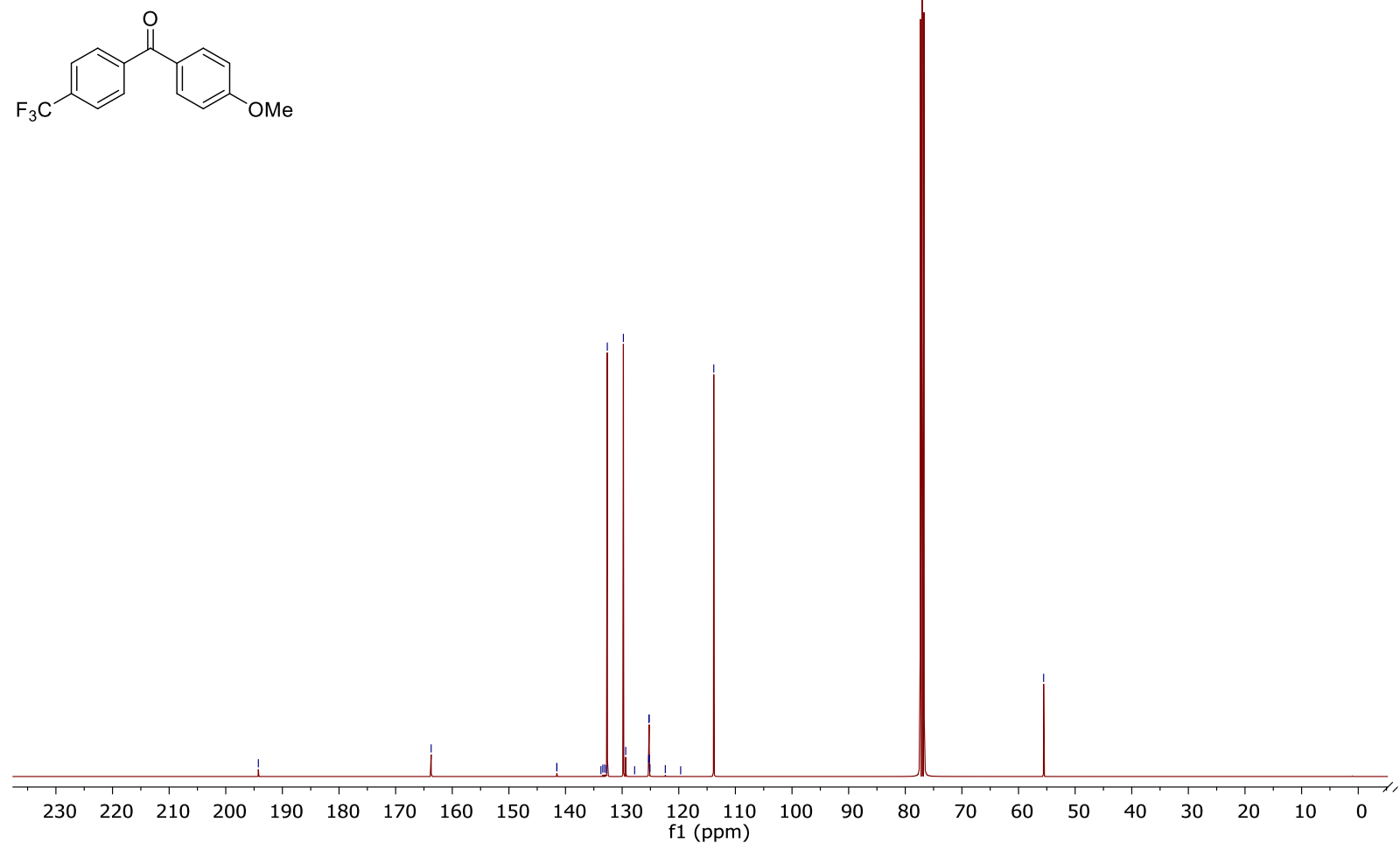

53 


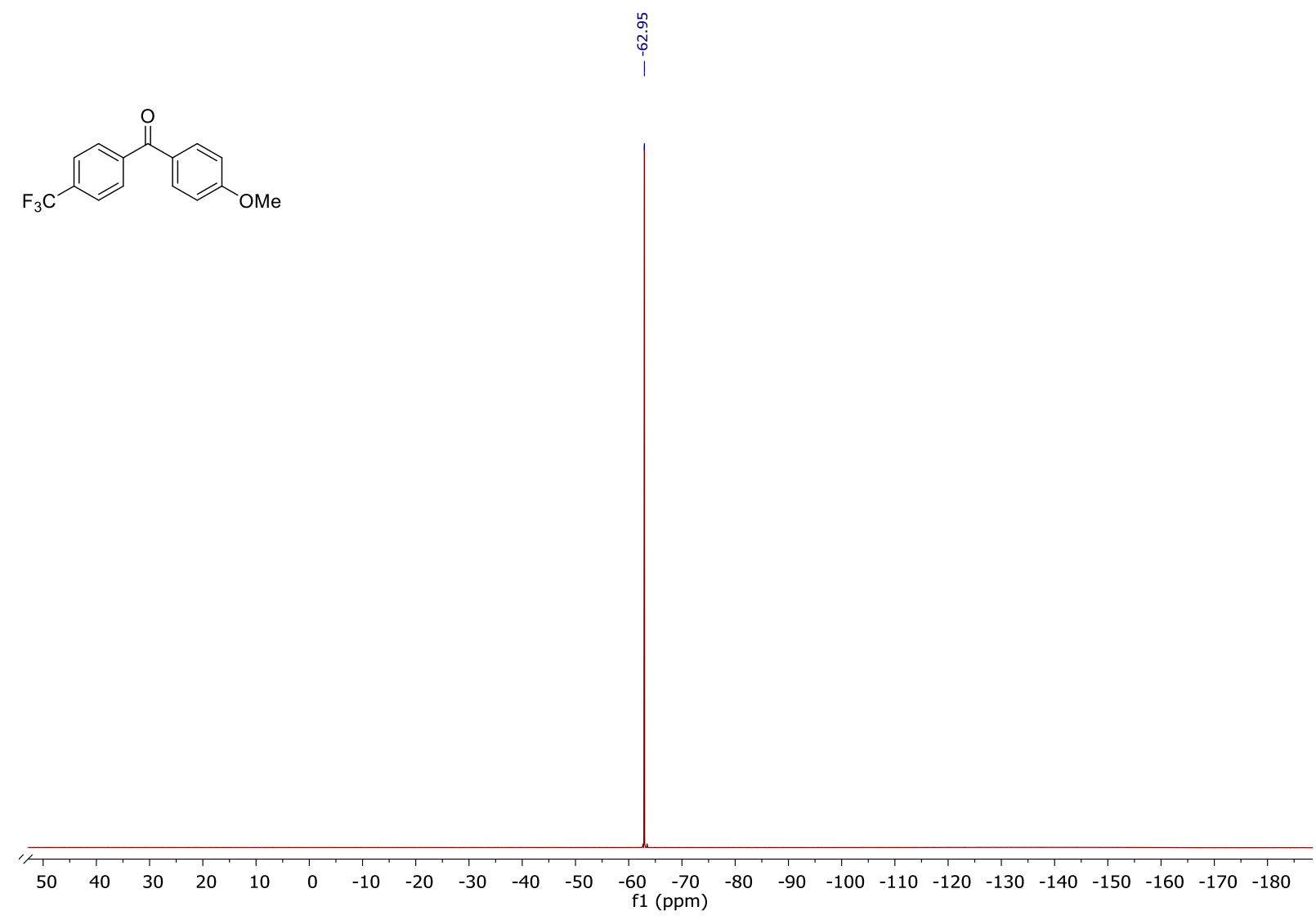

NMR-Spectra of (4-chlorophenyl)(4-fluoro-3-methylphenyl)methanone (5l)
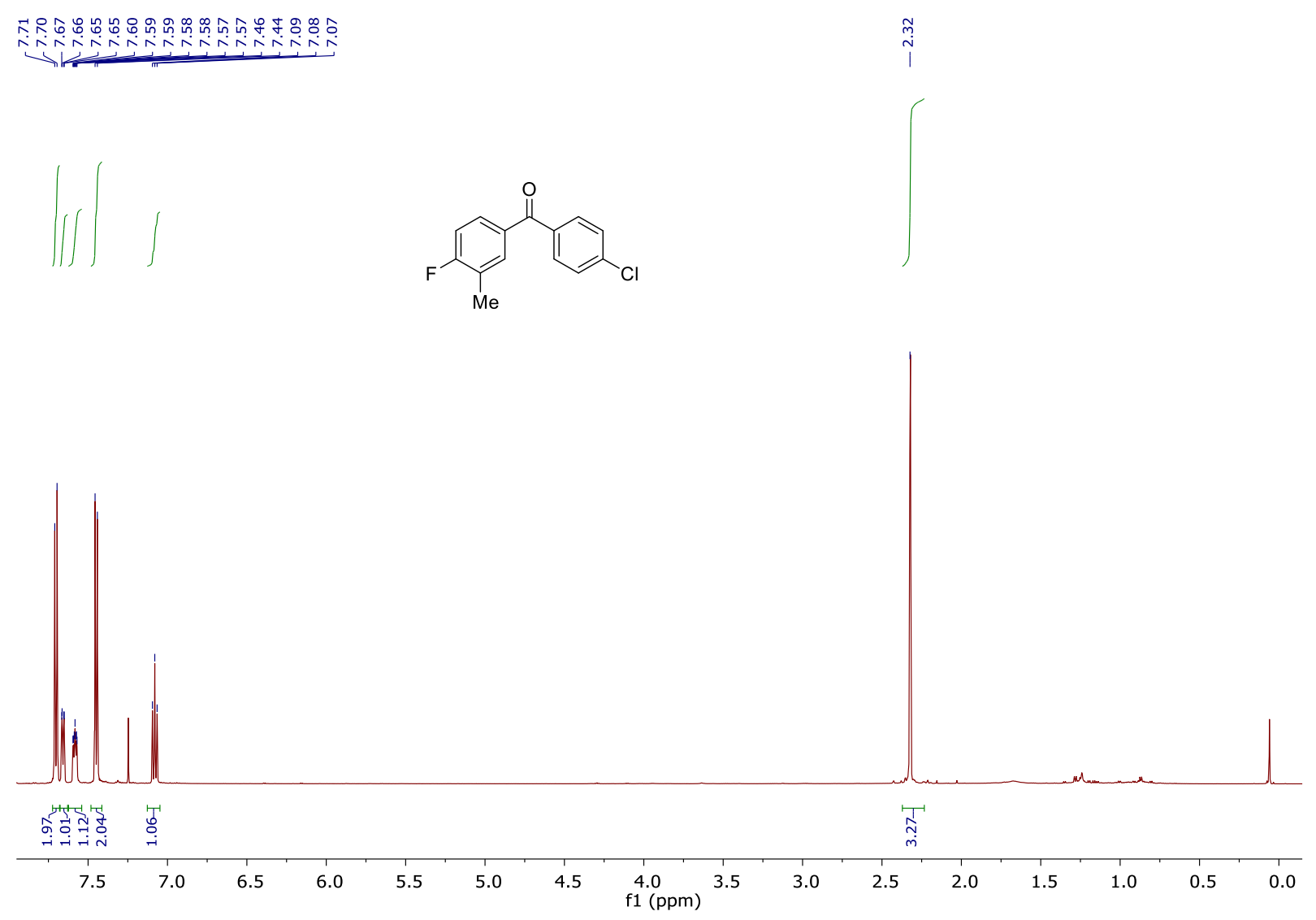


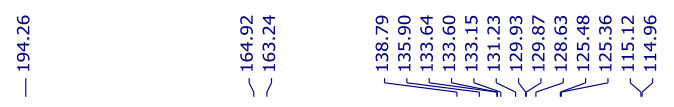

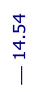
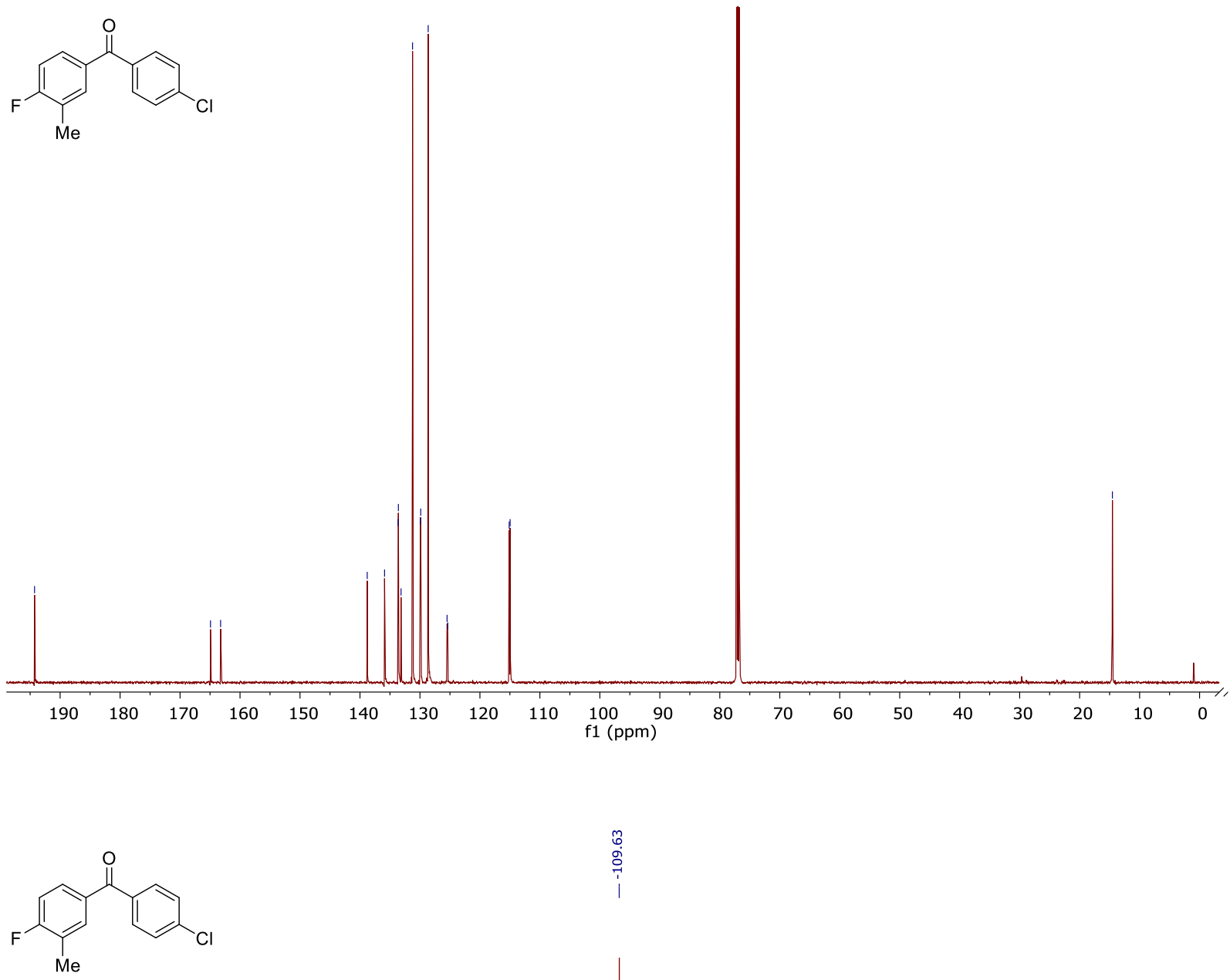

$\underset{\substack{0 \\ 0 \\ 0 \\ i}}{i}$

\begin{tabular}{llllllllllllllllll}
\hline \hline 50 & 30 & 10 & -10 & -30 & -50 & -70 & -90 & $\begin{array}{c}-110 \\
f 1(\mathrm{ppm})\end{array}$ & -130 & -150 & -170 & -190 & -210 & -230 & -250 & -270
\end{tabular}

55 
NMR-Spectra of (4-(tert-butyl)phenyl)(4-fluoro-3-methylphenyl)methanone (5m)

(1)
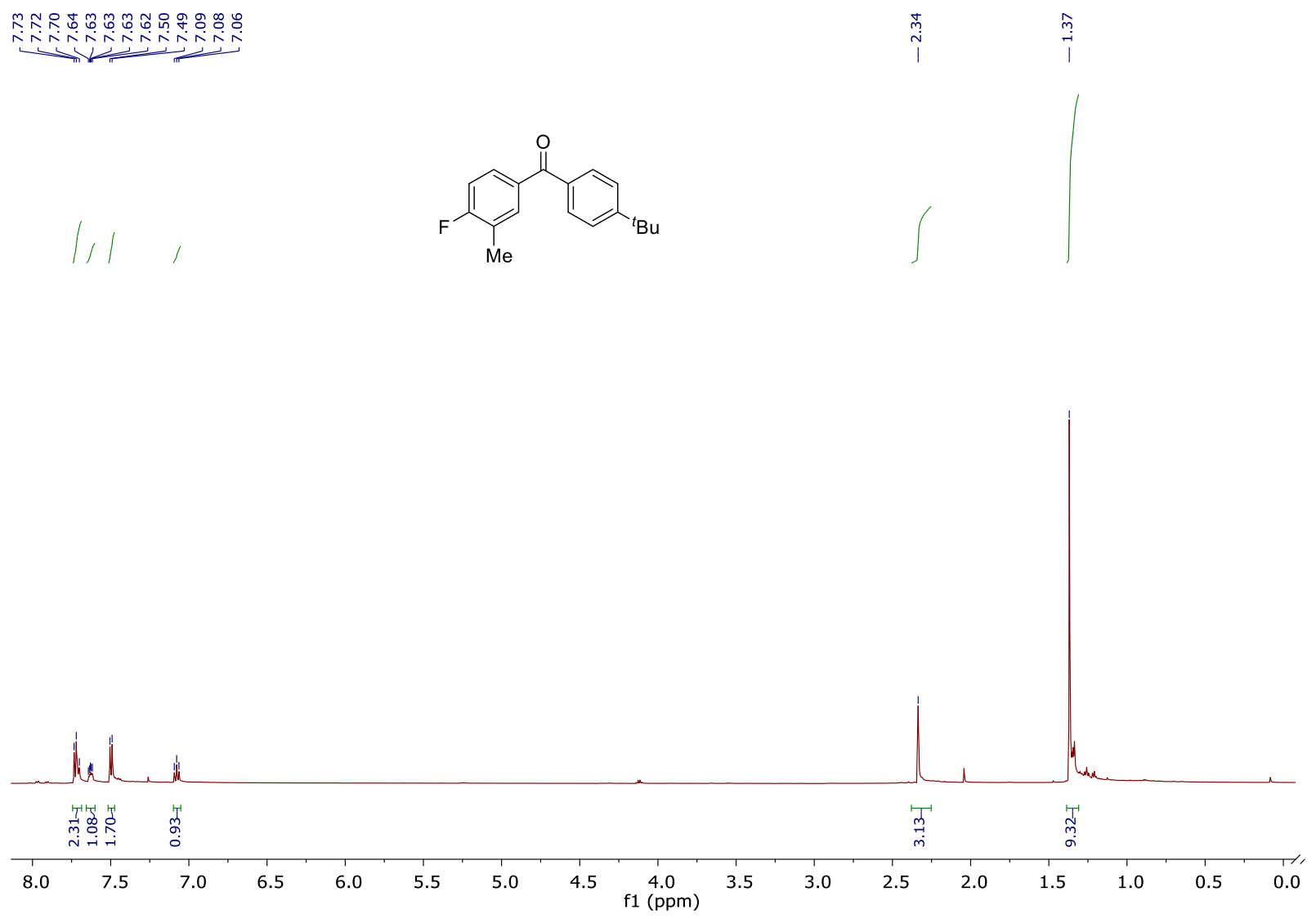

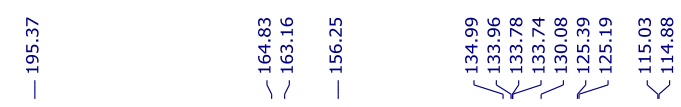
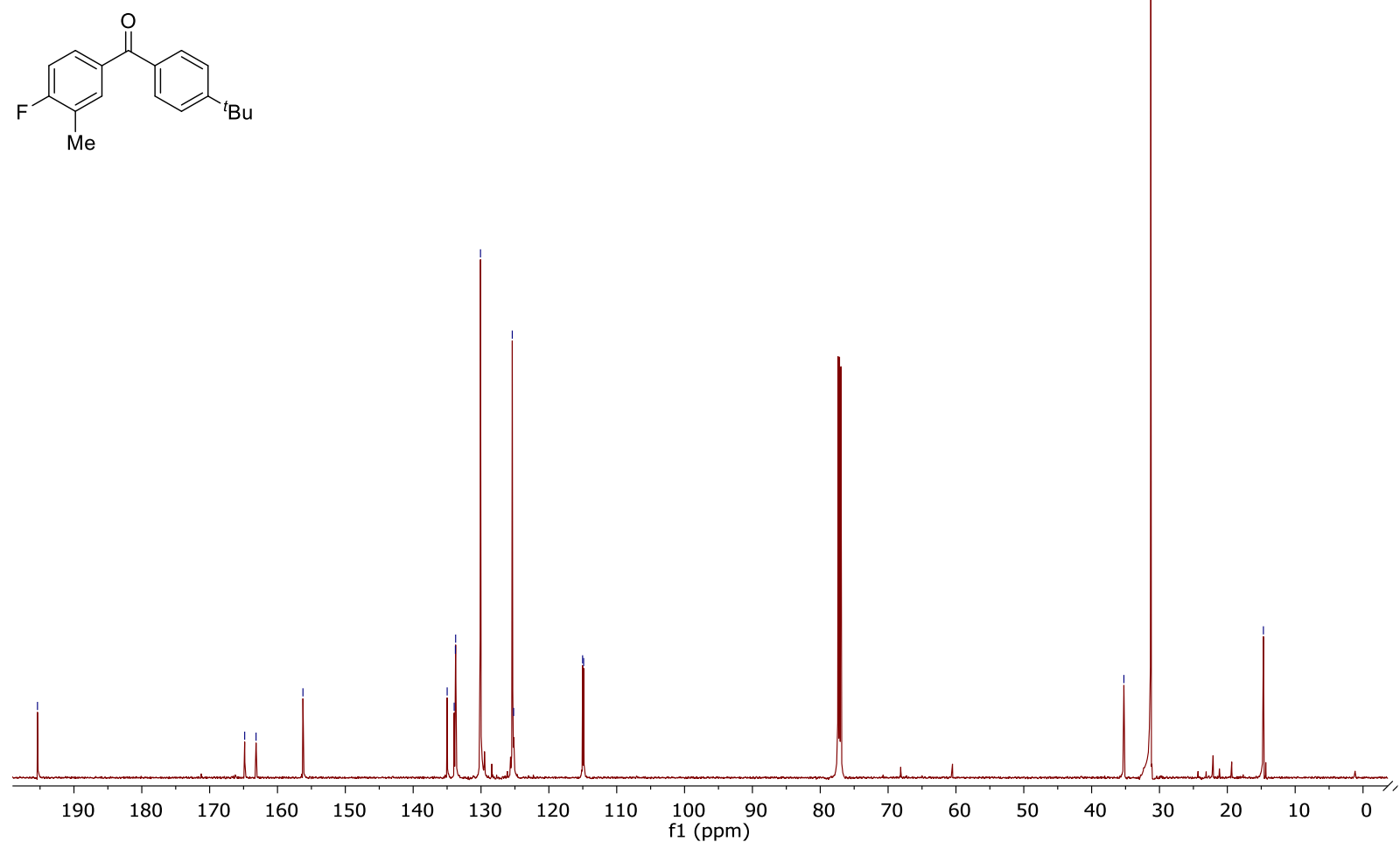

56 

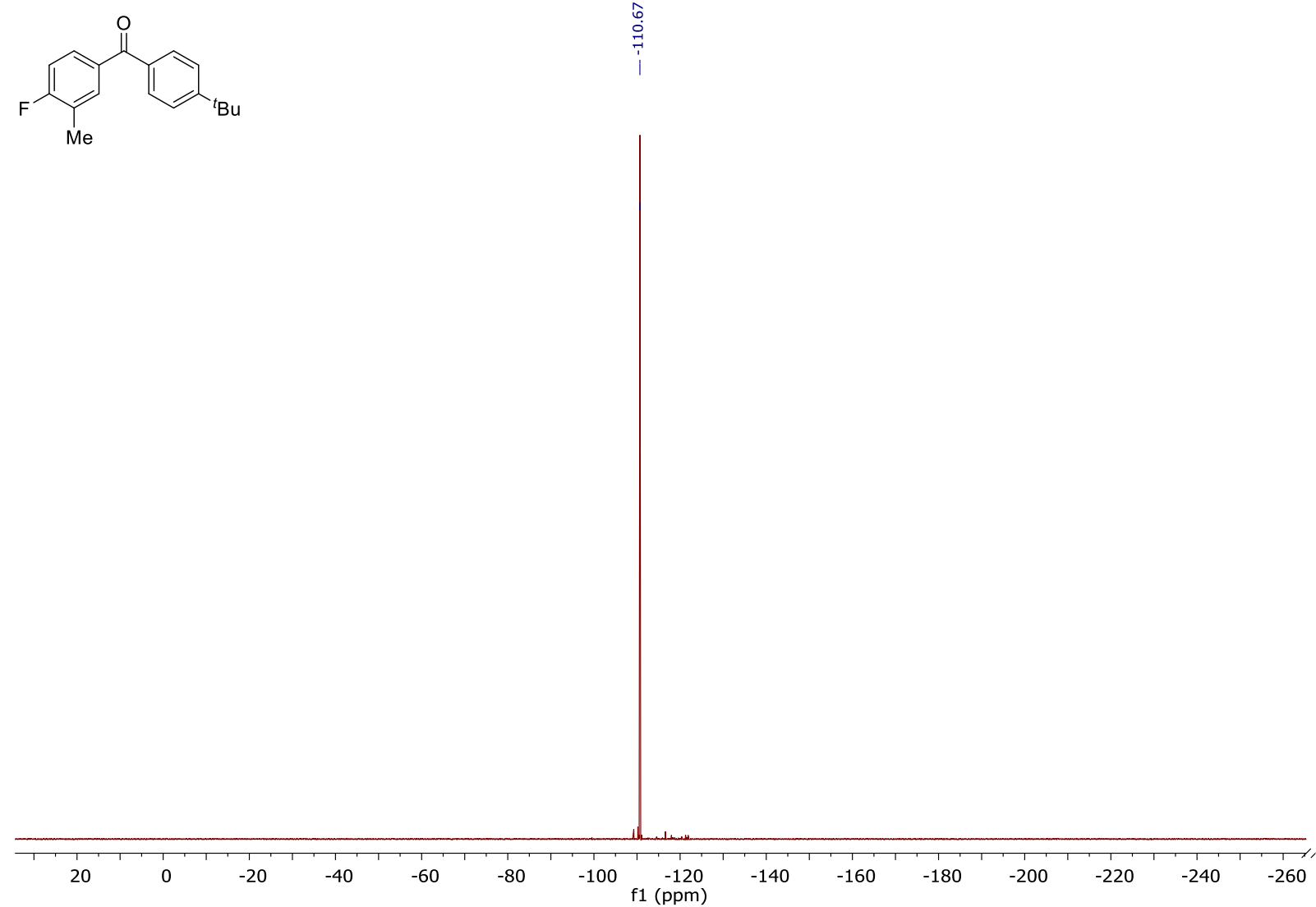

NMR-Spectra of (4-fluoro-3-methylphenyl)(4-methoxyphenyl)methanone (5n)
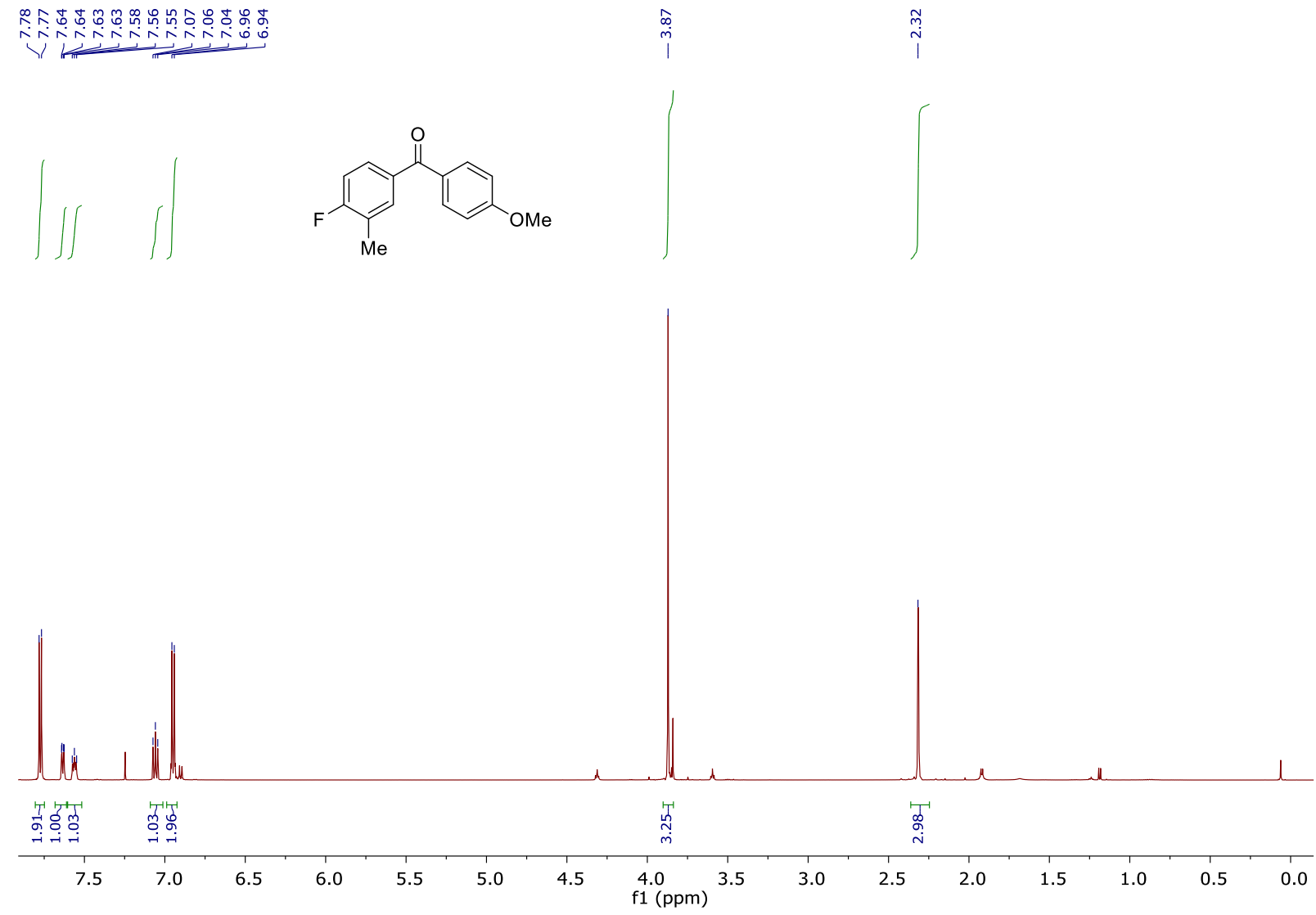


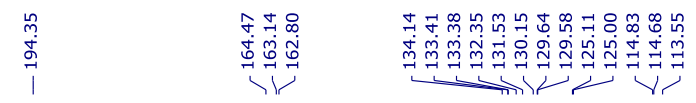

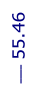

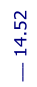
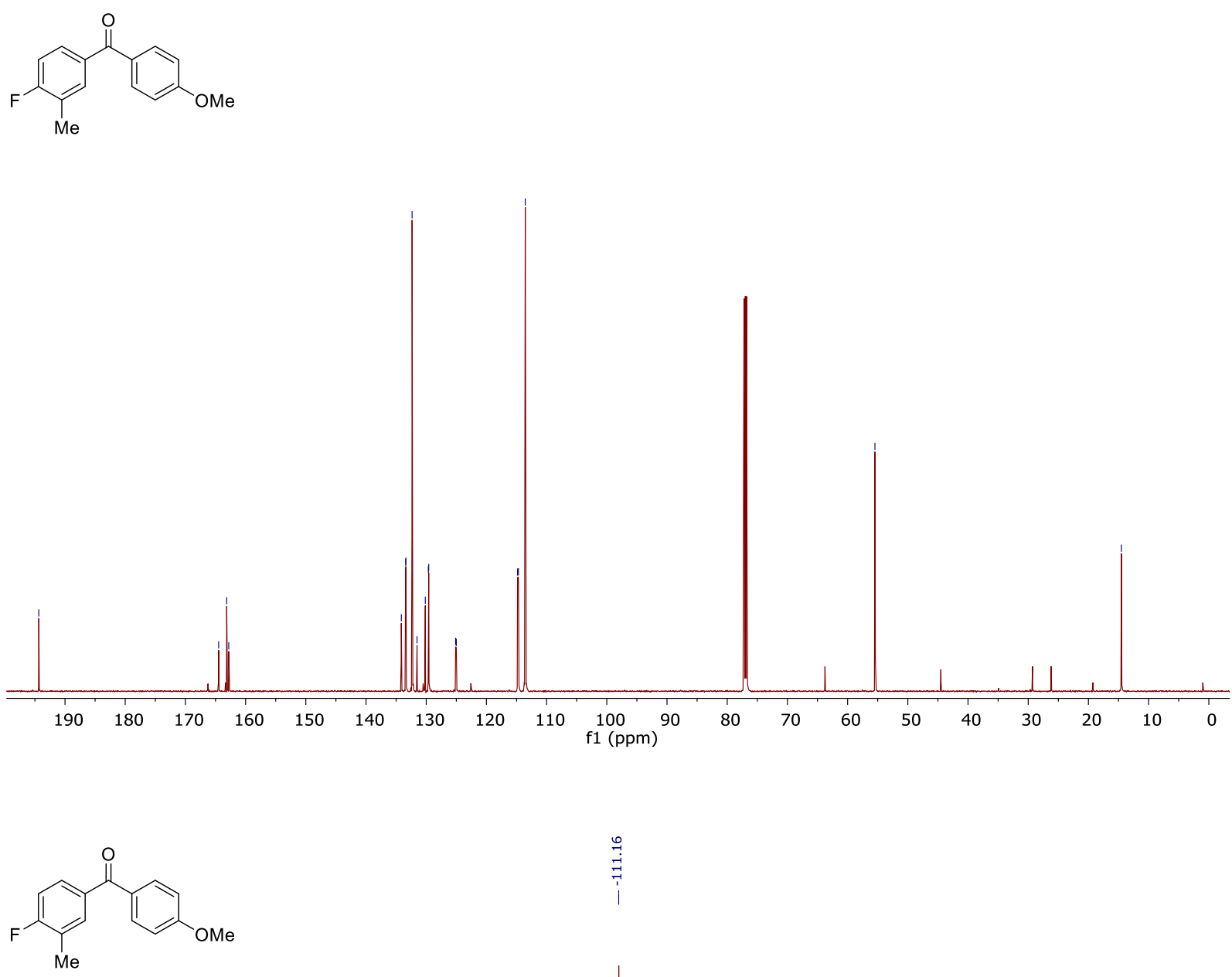



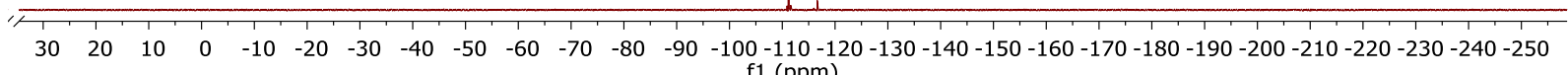

fl (ppm)

58 
NMR-Spectra of (4-chlorophenyl)(4-methoxyphenyl)methanone (5o)
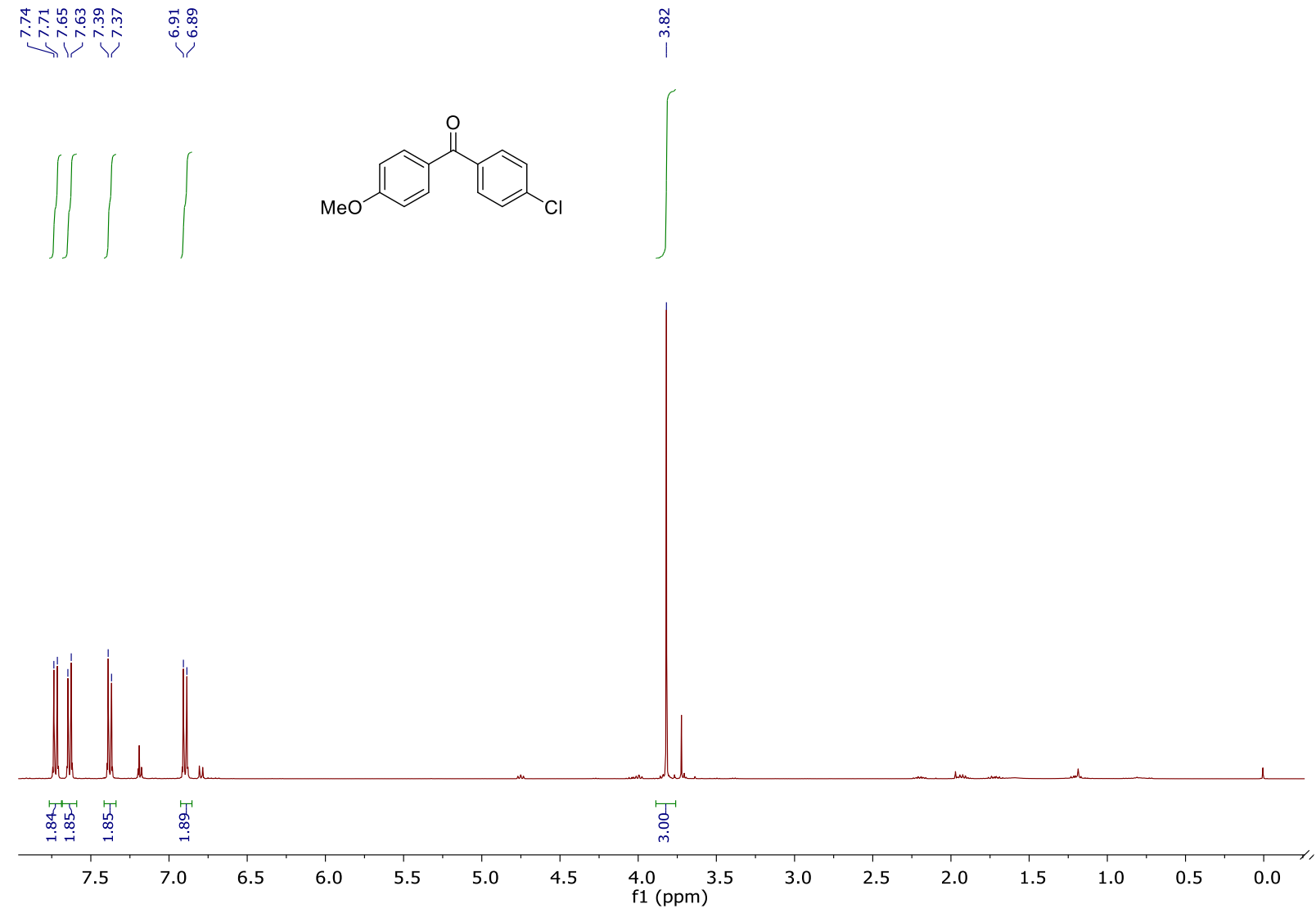

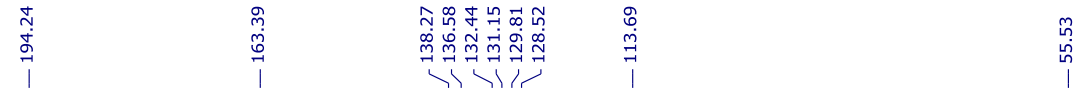<smiles>COc1ccc(C(=O)c2ccc(Cl)cc2)cc1</smiles>

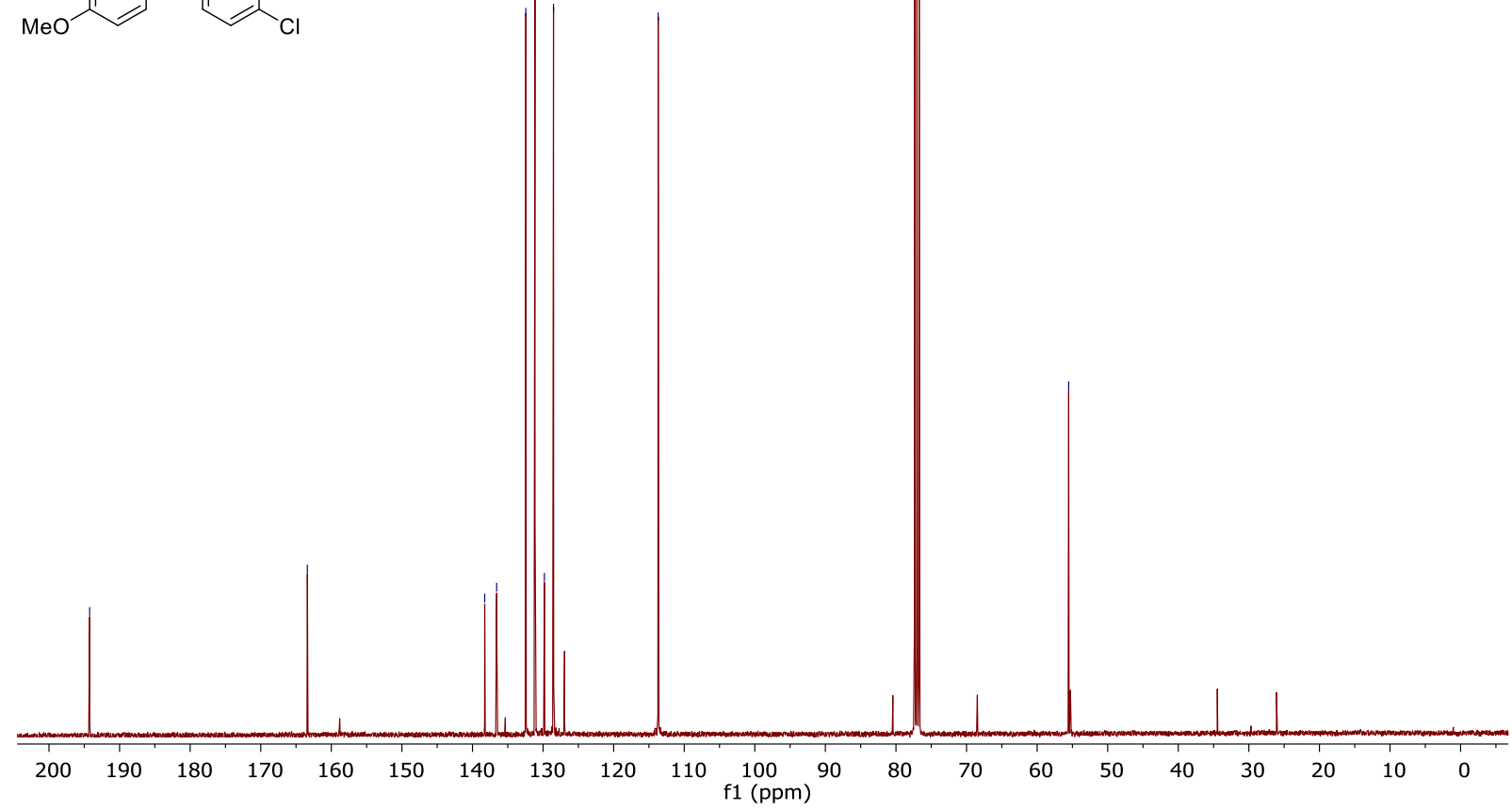


NMR-Spectra of (4-(tert-butyl)phenyl)(4-methoxyphenyl)methanone (5p)

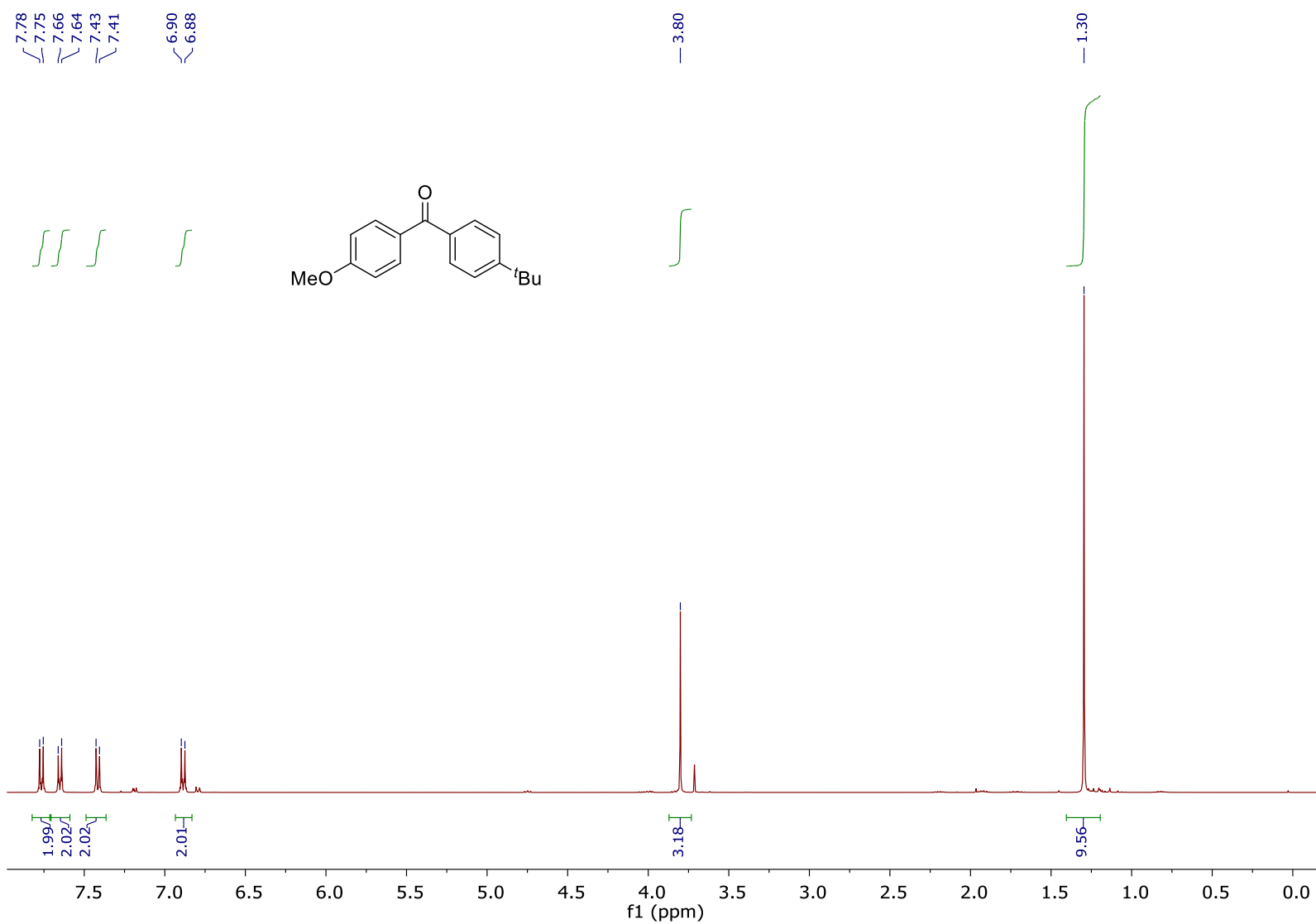

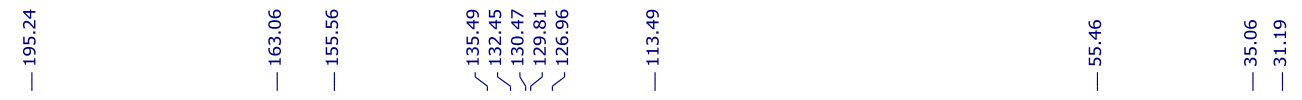<smiles>COc1ccc(C(=O)c2ccc(Br)cc2)cc1</smiles>

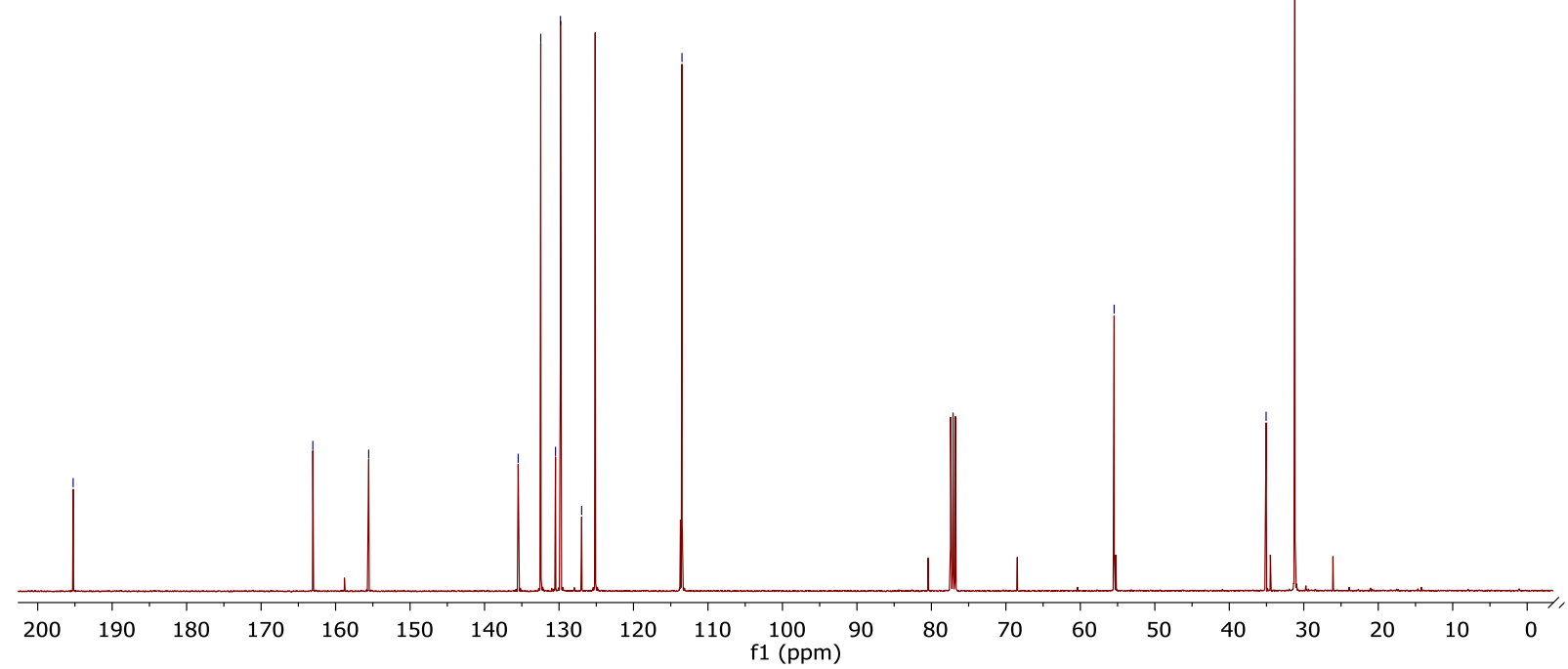


NMR-Spectra of (4-fluorophenyl)(4-methoxyphenyl)methanone (5q)

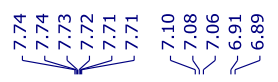

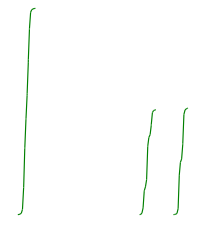

$\underset{\sim}{\sim}$

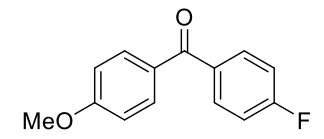

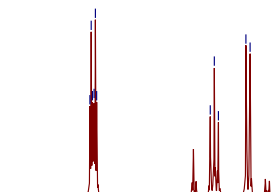

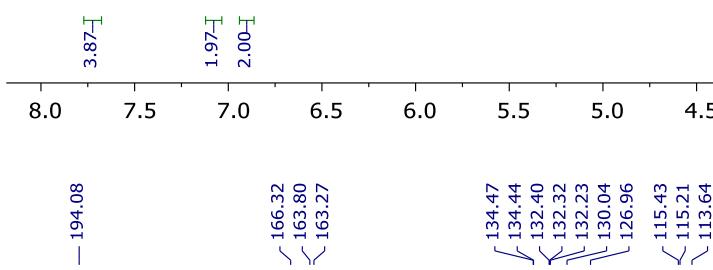

鬲

f1 4 (ppm)
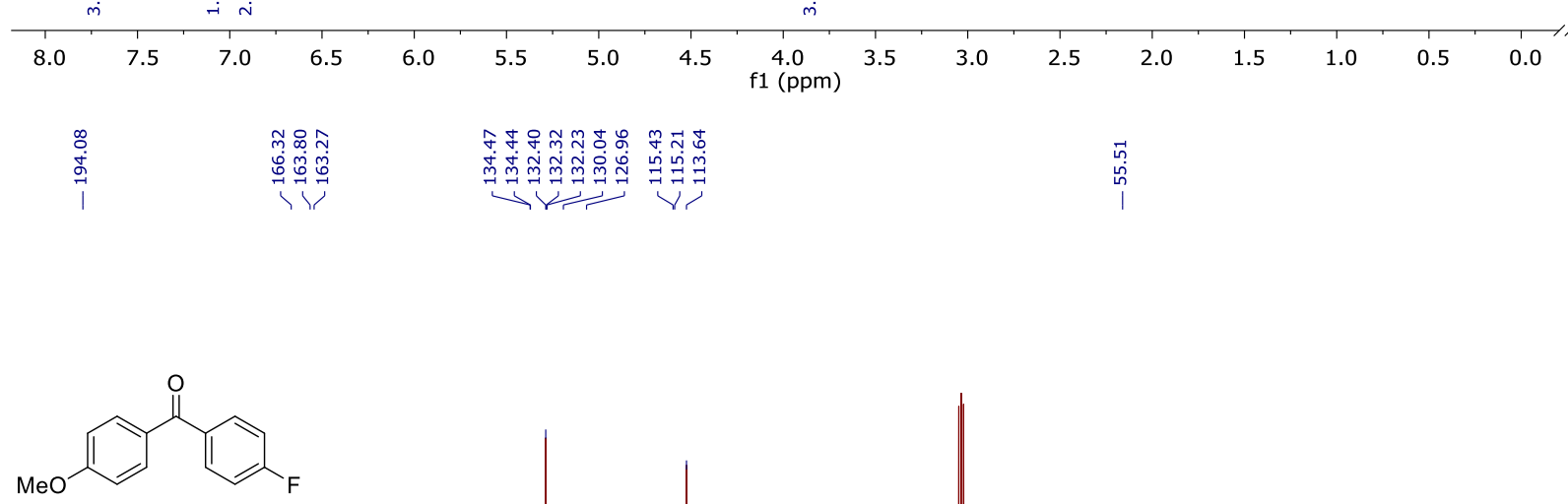

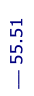

61 
<smiles>COc1ccc(C(=O)c2ccc(F)cc2)cc1</smiles> 
Table 1. Additional Screening of Additives for the Acylation of Benzylzinc Chloride (1a) with 4Chlorobenzoyl Chloride (2a)

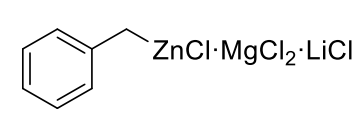

$1 \mathrm{a}$

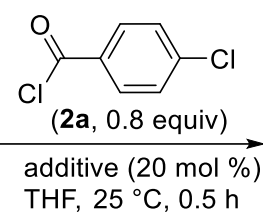

THF, $25^{\circ} \mathrm{C}, 0.5 \mathrm{~h}$

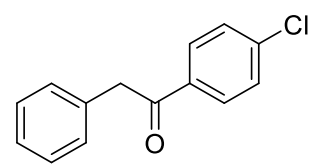

$3 a$

\begin{tabular}{ccc}
\hline entry & additive & yield $(\%)^{\mathrm{a}}$ \\
\hline 1 & 4 -DMAP & 55 \\
2 & $\mathrm{Sc}(\mathrm{OTf})_{3}$ & 54 \\
3 & $\mathrm{InCl}_{3}$ & traces \\
4 & $\mathrm{TiCl}_{4}$ & traces $^{\mathrm{b}}$ \\
5 & $\mathrm{BF}_{3} \cdot \mathrm{OEt}_{2}$ & 40 \\
6 & $\mathrm{LaCl}_{3} \cdot 2 \mathrm{LiCl}$ & traces
\end{tabular}

${ }^{\mathrm{a}}$ Isolated yield of pure product; ${ }^{\mathrm{b}}$ Almost quantitative amounts of homo-coupling were observed.

Table 2. Additional Screening of Polar Cosolvents for the Acylation of Benzylzinc Chloride (1a) with 4-Chlorobenzoyl Chloride (2a)

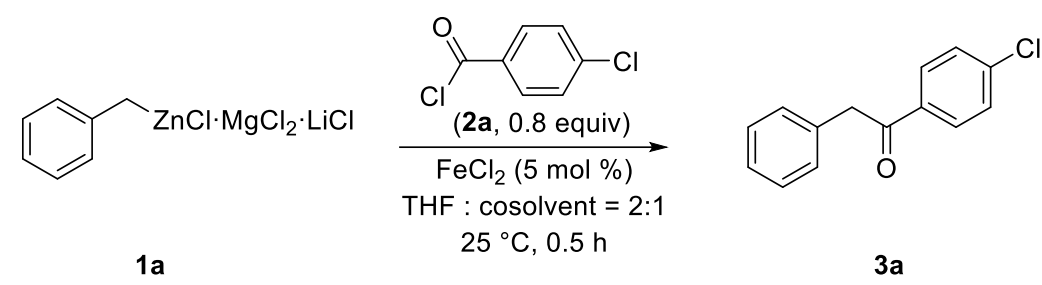

\begin{tabular}{ccc}
\hline entry & solvent ratio & yield $(\%)^{\mathrm{a}}$ \\
\hline 1 & THF : MeCN & 87 \\
2 & THF : NMP & 83 \\
3 & THF : DMPU & 68 \\
4 & THF : Dioxan & 64 \\
5 & THF : DMF & 29 \\
6 & THF : DMAc & traces
\end{tabular}

${ }^{\mathrm{a}}$ Isolated yield of pure product. 
Table 3. Additional Screening of Catalysts for the Acylation of (4-(ethoxycarbonyl)phenyl)zinc(II) chloride (4a) with 4-Chlorobenzoyl Chloride (2a)

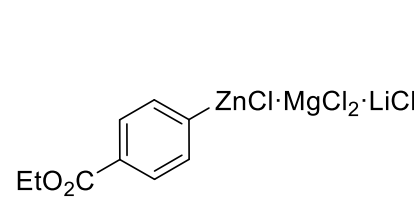

$4 a$

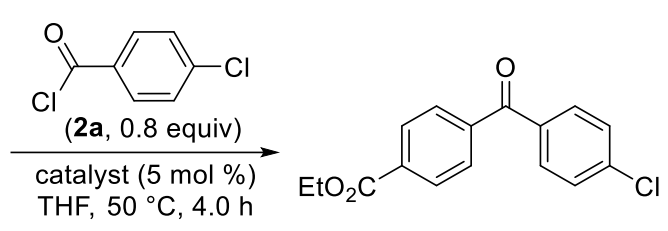

$5 a$

\begin{tabular}{ccc}
\hline entry & catalyst & yield $(\%)^{\mathrm{a}}$ \\
\hline 1 & - & 34 \\
2 & $\mathrm{Fe}(\mathrm{OTf})_{3}$ & 34 \\
3 & $\mathrm{Fe}(\mathrm{acac})_{3}$ & 36 \\
4 & $\mathrm{FeBr}_{3}$ & 53 \\
5 & $\mathrm{Fe}(\mathrm{acac})_{2}$ & 60 \\
6 & $\mathrm{FeBr}_{2}$ & 56 \\
7 & $\mathrm{FeCl}_{2}$ & 62
\end{tabular}

${ }^{\mathrm{a}}$ Isolated yield of pure product.

Table 4. Additional Screening of Polar Cosolvents for the Acylation of (4-(ethoxycarbonyl)phenyl)zinc(II) chloride (4a) with 4-Chlorobenzoyl Chloride (2a)

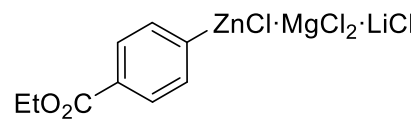

$4 a$

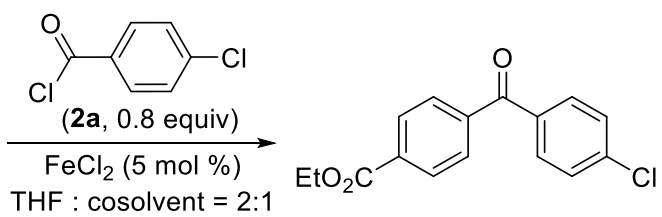

$50{ }^{\circ} \mathrm{C}, 4.0 \mathrm{~h}$

$5 a$

\begin{tabular}{ccc}
\hline entry & solvent ratio & yield $(\%)^{\mathrm{a}}$ \\
\hline 1 & THF : DMF & 38 \\
2 & THF : NMP & 42 \\
3 & THF : DMPU & 45 \\
4 & THF : MeCN & 60 \\
5 & THF : Dioxan & 64 \\
6 & THF : DMAc & 64
\end{tabular}

\footnotetext{
${ }^{\mathrm{a}}$ Isolated yield of pure product.
} 


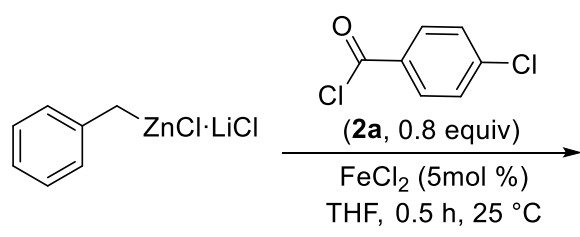

$1 \mathrm{a}$<smiles>FC(F)(F)c1cccc(C[Ge]Cl)c1</smiles>

$1 \mathrm{~b}$<smiles>FC(F)(F)c1cccc(C[Ge]Cl)c1</smiles>

$1 b$<smiles>Fc1cccc(C[Ge]Cl)c1</smiles>

$1 c$<smiles>CCOC(=O)c1ccc([Ga]Cl)cc1</smiles>

$4 a$<smiles>CCOC(=O)c1ccc([Ga]Cl)cc1</smiles>

$4 a$

$1 c$
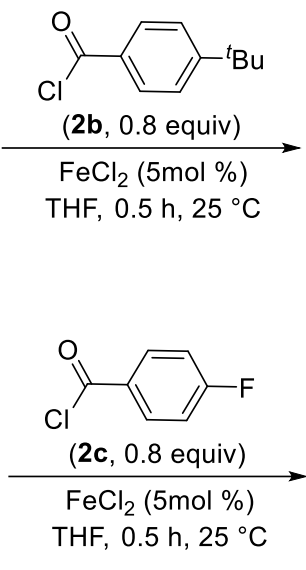<smiles>O=C(Cc1ccccc1)c1ccc(Cl)cc1</smiles>

3a $(72 \%)(90 \%)^{a}$<smiles>CC(C)(C)c1ccc(C(=O)Cc2cccc(C(F)(F)F)c2)cc1</smiles>

3b $(66 \%)(65 \%)^{a}$<smiles>O=C(Cc1cccc(C(F)(F)F)c1)c1ccc(Cl)cc1</smiles>

3c $(71 \%)(70 \%)^{a}$<smiles>CC(C)(C)c1ccc(C(=O)Cc2cccc(F)c2)cc1</smiles>

3d $(71 \%)(88 \%)^{a}$<smiles>O=C(Cc1cccc(F)c1)c1ccc(F)cc1</smiles>

3e $(60 \%)(74 \%)^{a}$
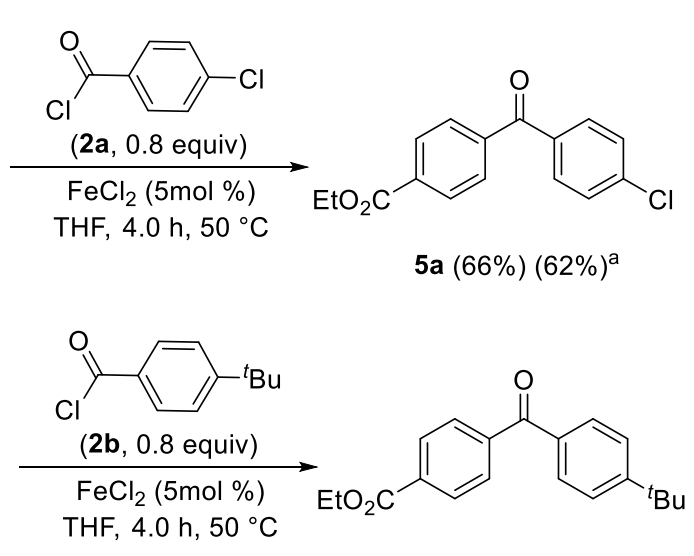

5b $(68 \%)(65 \%)^{a}$

Scheme 1: Iron-catalyzed acylations of aryl- and benzyl-halides (1a-c, 4a), prepared by the direct oxidative insertion of zinc dust in the presence of $\mathrm{LiCl}$, with acid chlorides (2a-c). ${ }^{\text {a }}$ Yields refer to isolated yields of pure products obtained by using the in situ transmetalated organozinc species. 


\section{Preliminary Experiments towards Iron-Catalyzed Acylation of Alkylzinc Halides (6a,b)}

\section{Preparation of cyclohexylzinc(II) iodide (6a)}

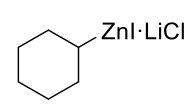

A dry and argon-flushed Schlenk-tube, equipped with a magnetic stirring bar and a rubber septum, was charged with $\mathrm{LiCl}\left(0.95 \mathrm{~g}, 22.5 \mathrm{mmol}, 1.50\right.$ equiv) and heated up to ca. $450{ }^{\circ} \mathrm{C}$ for $5 \mathrm{~min}$ under high vacuum using a heat gun. After cooling to room temperature under vigorous stirring, $\mathrm{Zn}$ dust $(2.94 \mathrm{~g}$, $45.0 \mathrm{mmol}, 3.00$ equiv) was added and heated up to ca. $450{ }^{\circ} \mathrm{C}$ for $5 \mathrm{~min}$ one more time. Freshly distilled THF (15 mL), trimethylsilyl chloride (5 mol \%) and 1,2-dibromoethane (5 mol \%) were added and the suspension was heated shortly to reflux. Iodocyclohexane ( $3.15 \mathrm{~g}, 1.94 \mathrm{~mL}, 15.0 \mathrm{mmol}, 1.00$ equiv) was added dropwise at room temperature and the reaction mixture was stirred for additional $30 \mathrm{~min}$. The completion of the metalation was monitored by GC-analysis of hydrolyzed and iodolyzed aliquots. When the oxidative insertion was complete, the solution was separated from the zinc dust via a syringe equipped with a filter and transferred to another pre-dried and argon-flushed Schlenk-tube. The concentration of cyclohexylzinc(II) iodide (6a) was determined by titration with iodine in THF $(0.80 \mathrm{M})$.

\section{Preparartion of butylzinc(II) chloride (6b)}

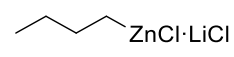

A dry and argon-flushed Schlenk-tube, equipped with a magnetic stirring bar and a rubber septum, was charged with $n \mathrm{BuLi}(3.85 \mathrm{~mL}, 2.60 \mathrm{M}$ in hexane, $10.0 \mathrm{mmol})$ and cooled to $-40{ }^{\circ} \mathrm{C}$. $\mathrm{ZnCl}_{2}(12.0 \mathrm{~mL}$, $1.00 \mathrm{M}$ in THF, $12.0 \mathrm{mmol}$ ) was added and to resulting solution was stirred for additional $10 \mathrm{~min}$. The concentration of butylzinc(II) chloride (6b) was determined by titration with iodine in $\operatorname{THF}(0.54 \mathrm{M})$.

Typical Procedure for the Iron-Catalyzed Acylation of Alkylzinc Halides (6a,b) with Acid Chlorides (TP 5):

A dry and argon-flushed Schlenk-tube, equipped with a magnetic stirring bar and a rubber septum, was charged with $\mathrm{FeCl}_{2}$ (6.34 mg, $0.05 \mathrm{mmol}, 5.0 \mathrm{~mol} \%$ ), the selected acid chloride (1.00 mmol, 1.00 equiv) and freshly distilled THF $(1 \mathrm{~mL})$. The corresponding prior prepared alkylzinc halide solution (1.50 mmol, 1.50 equiv) was dropwise added and the reaction mixture was stirred for a given time at room temperature. Subsequently, the reaction mixture was quenched with a saturated aqueous $\mathrm{NH}_{4} \mathrm{Cl}$ solution and the aqueous layer was extracted with EtOAc $(3 \times 75 \mathrm{~mL})$. The combined organic layers were dried over $\mathrm{MgSO}_{4}$, filtered and concentrated under reduced pressure. Purification of the crude products by flash column chromatography afforded the desired products. 

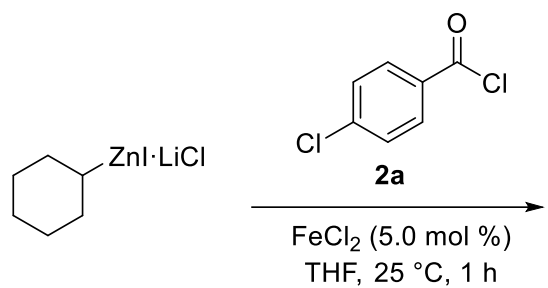<smiles>O=C(Cl)c1ccc(Cl)cc1</smiles>

6a 1.50 equiv, $0.80 \mathrm{M}$

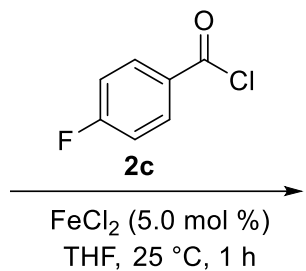

$7 \mathrm{a}(40 \%)(40 \%)^{\mathrm{a}}$

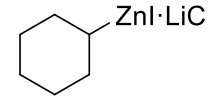

$\mathrm{THF}, 25^{\circ} \mathrm{C}, 1 \mathrm{~h}$

6a 1.50 equiv, $0.80 \mathrm{M}$

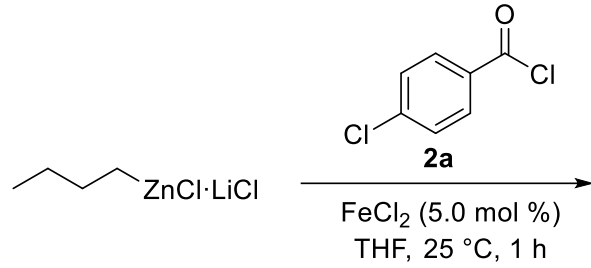

$7 b(49 \%)$

6b 1.50 equiv, $0.54 \mathrm{M}$

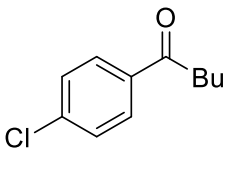

7c $(60 \%)(37 \%)^{a}$

Scheme 2: Iron-catalyzed acylations of alkylzinc halides $(\mathbf{6 a}, \mathbf{b})$ with acid chlorides $(\mathbf{2 a}, \mathbf{c}) \cdot{ }^{\text {a }}$ A solvent mixture of THF:NMP $=1: 1$ was used, see reference $8 b$ in the manuscript.

\section{Preparation of cyclohexyl(4-chlorophenyl)methanone (7a)}<smiles>O=C(O)c1ccc(Cl)cc1</smiles>

According to TP5, $\mathrm{FeCl}_{2} 6.34 \mathrm{mg}, 0.05 \mathrm{mmol}, 5.0 \mathrm{~mol} \%$ ), 4-chlorobenzoyl chloride (2a, $175 \mathrm{mg}$, $0.13 \mathrm{~mL}, 1.00 \mathrm{mmol}, 1.00$ equiv) and freshly distilled THF (1.0 mL) were used. The cyclohexylzinc(II) iodide (6a, $1.88 \mathrm{~mL}, 1.50 \mathrm{mmol}, 0.80 \mathrm{M}$ in THF, 1.50 equiv) was added dropwise and the reaction mixture was stirred for $1 \mathrm{~h}$ at room temperature. The reaction was quenched with a saturated aqueous $\mathrm{NH}_{4} \mathrm{Cl}$ solution and the aqueous layer was extracted with EtOAc $(3 \times 75 \mathrm{~mL})$. The combined organic layers were dried over $\mathrm{MgSO}_{4}$, filtered and concentrated under reduced pressure. The crude product was purified by flash column chromatography $\left(\mathrm{SiO}_{2}, i\right.$-hexane $:$ EtOAc $\left.=99: 1, \mathrm{R}_{\mathrm{f}}=0.40\right)$ leading to the product $7 \mathbf{a}(88 \mathrm{mg}, 0.40 \mathrm{mmol}, 40 \%)$ as a pale yellow oil.

The analytical data of product $7 \mathbf{a}$ is consistent with the data given in the literature. ${ }^{1}$

${ }^{1}$ H-NMR (400 MHz, CDCl 3 , ppm) $\delta=7.89(\mathrm{~d}, J=8.4 \mathrm{~Hz}, 2 \mathrm{H}), 7.44(\mathrm{~d}, J=8.4 \mathrm{~Hz}, 2 \mathrm{H}), 3.25-3.20(\mathrm{~m}$, $1 \mathrm{H}), 1.88-1.84(\mathrm{~m}, 4 \mathrm{H}), 1.77-1.74(\mathrm{~m}, 1 \mathrm{H}), 1.56-1.24(\mathrm{~m}, 5 \mathrm{H})$ 
${ }^{13}$ C-NMR (100 MHz, CDCl $\left.\mathbf{3}, \mathbf{p p m}\right) \delta=202.4,139.3,134.5,129.7,128.9,45.6,29.4,25.8,25.7$.

IR $\left(\mathbf{A T R}, \mathbf{c m}^{-1}\right) \tilde{\mathrm{v}}=2929,2854,1714,1681,1590,1572,1487,1449,1400,1371,1315,1274,1249$, 1206, 1171, 1114, 1104, 1090, 1037, 1013, 974, 893, 834, 760, 738, 685.

MS (EI, 70 eV , \%) m/z = 222 (5), 187 (11), 157 (16), 141 (28), 139 (100), 111 (16), 82 (14), 67 (10), $55(10)$.

HRMS (EI, $70 \mathbf{~ e V}$ ) m/z: calc. for $\mathbf{C}_{\mathbf{1 3}} \mathbf{H}_{15} \mathbf{C l O}$ : 222.0811; found 222.0814 .

\section{Preparation of cyclohexyl(4-fluorophenyl)methanone (7b)}

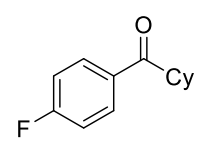

According to TP5, $\mathrm{FeCl}_{2} 6.34 \mathrm{mg}, 0.05 \mathrm{mmol}, 5.0 \mathrm{~mol} \%$ ), 4-fluorobenzoyl chloride (2c, $159 \mathrm{mg}$, $0.12 \mathrm{~mL}, 1.00 \mathrm{mmol}, 1.00$ equiv) and freshly distilled THF (1.0 mL) were used. The cyclohexylzinc(II) iodide (6a, $1.88 \mathrm{~mL}, 1.50 \mathrm{mmol}, 0.80 \mathrm{M}$ in THF, 1.50 equiv) was added dropwise and the reaction mixture was stirred for $1 \mathrm{~h}$ at room temperature. The reaction was quenched with a saturated aqueous $\mathrm{NH}_{4} \mathrm{Cl}$ solution and the aqueous layer was extracted with EtOAc $(3 \times 75 \mathrm{~mL})$. The combined organic layers were dried over $\mathrm{MgSO}_{4}$, filtered and concentrated under reduced pressure. The crude product was purified by flash column chromatography $\left(\mathrm{SiO}_{2}\right.$, $i$-hexane : EtOAc $\left.=99: 1, \mathrm{R}_{\mathrm{f}}=0.44\right)$ leading to the product $7 \mathbf{b}$ (101 mg, $0.49 \mathrm{mmol}, 49 \%)$ as a pale yellow oil.

The analytical data of product $\mathbf{7 b}$ is consistent with those given in the literature. ${ }^{1}$

${ }^{1}$ H-NMR (400 MHz, CDCl 3 , ppm) $\delta=8.00-7.97(\mathrm{~m}, 2 \mathrm{H}), 7.14(\mathrm{t}, J=8.8 \mathrm{~Hz}, 2 \mathrm{H}), 3.26-3.19(\mathrm{~m}, 1 \mathrm{H})$, $1.90-1.85(\mathrm{~m}, 4 \mathrm{H}), 1.77-1.73(\mathrm{~m}, 1 \mathrm{H}), 1.55-1.26(\mathrm{~m}, 5 \mathrm{H})$.

${ }^{13} \mathrm{C}-\mathrm{NMR}\left(\mathbf{1 0 0} \mathrm{MHz}, \mathrm{CDCl}_{3}, \mathbf{p p m}\right) \delta=202.2,165.6\left(\mathrm{~d},{ }^{1} \mathrm{~J}(\mathrm{C}, \mathrm{F})=252 \mathrm{~Hz}\right), 132.6\left(\mathrm{~d},{ }^{4} J(\mathrm{C}, \mathrm{F})=2.4 \mathrm{~Hz}\right)$, $130.9\left(\mathrm{~d},{ }^{3} J(\mathrm{C}, \mathrm{F})=8.7 \mathrm{~Hz}\right), 115.7\left(\mathrm{~d},{ }^{2} J(\mathrm{C}, \mathrm{F})=22 \mathrm{~Hz}\right), 45.6,29.4,25.9,25.8$.

${ }^{19}$ F-NMR (376 MHz, CDCl 3 , ppm) $\delta=-106.0$.

IR $\left(\mathbf{A T R}, \mathbf{c m}^{-1}\right) \tilde{\mathrm{v}}=2929,2855,1714,1680,1597,1505,1450,1410,1372,1313,1277,1234,1206$, 1154, 1113, 1091, 1013, 975, 894, 842, 768, 740, 675. 
MS (EI, 70 eV, \%) m/z = 207 (14), 206 (4), 124 (10), 123 (100), 109 (16), 95 (12), 83 (15), 55 (16), 41 (10).

HRMS (EI, 70 eV) m/z: calc. for $\mathbf{C}_{\mathbf{1 3}} \mathbf{H}_{\mathbf{1 5}} \mathbf{F O}$ : 206.1107; found 206.1108 .

\section{Preparation of 1-(4-chlorophenyl)pentan-1-one (7c)}

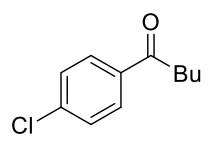

Based on TP5, $\mathrm{FeCl}_{2} 6.34 \mathrm{mg}, 0.05 \mathrm{mmol}, 5.0 \mathrm{~mol} \%$ ), 4-chlorobenzoyl chloride (2a, $175 \mathrm{mg}, 0.13 \mathrm{~mL}$, $1.00 \mathrm{mmol}, 1.00$ equiv) and freshly distilled THF (1.0 mL) were used. The butylzinc(II) chloride (6b, $2.78 \mathrm{~mL}, 1.50 \mathrm{mmol}, 0.54 \mathrm{M}$ in THF, 1.50 equiv) was added dropwise and the reaction mixture was stirred for $1 \mathrm{~h}$ at room temperature. The reaction was quenched with a saturated aqueous $\mathrm{NH}_{4} \mathrm{Cl}$ solution and the aqueous layer was extracted with EtOAc $(3 \times 75 \mathrm{~mL})$. The combined organic layers were dried over $\mathrm{MgSO}_{4}$, filtered and concentrated under reduced pressure. The crude product was purified by flash column chromatography $\left(\mathrm{SiO}_{2}, i\right.$-hexane $:$ EtOAc $\left.=99: 1, \mathrm{R}_{\mathrm{f}}=0.55\right)$ leading to the product $7 \mathrm{c}(118 \mathrm{mg}$, $0.60 \mathrm{mmol}, 60 \%)$ as a colorless oil.

The analytical data of product $\mathbf{7 c}$ is consistent with those given in the literature. ${ }^{2}$

${ }^{1} \mathbf{H}-\mathrm{NMR}\left(400 \mathrm{MHz}, \mathbf{C D C l}_{3}\right.$, ppm) $\delta=7.90-7.87(\mathrm{dt}, J=2.4,8.8 \mathrm{~Hz}, 2 \mathrm{H}), 7.43-7.40(\mathrm{dt}, J=2.4,8.8$ $\mathrm{Hz}, 2 \mathrm{H}), 2.94-2.91(\mathrm{t}, J=7.2 \mathrm{~Hz}, 2 \mathrm{H}), 1.74-1.67(\mathrm{~m}, 2 \mathrm{H}), 1.44-1.35(\mathrm{~m}, 2 \mathrm{H}), 0.96-0.93(\mathrm{t}, J=7.2 \mathrm{~Hz}$, $3 \mathrm{H})$.

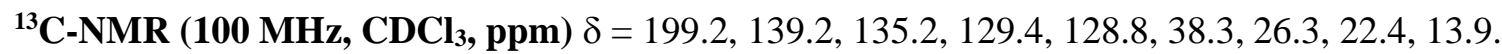

IR $\left(\mathbf{A T R}, \mathbf{c m}^{-1}\right) \tilde{\mathrm{v}}=2958,2932,2873,1722,1687,1590,1572,1488,1400,1271,1205,1174,1090$, 1012, 968, 838, 810, 793, 760, 733, 685.

MS (EI, 70 eV, \%) m/z = 196 (1), 156 (11), 154 (26), 141 (30), 139 (100), 111 (20), 75 (10).

HRMS (EI, 70 eV) m/z: calc. for $\mathbf{C}_{\mathbf{1 1}} \mathbf{H}_{\mathbf{1 3}}$ ClO: 196.0655; found 196.0642 .

\section{References}

(1) Zhang, X.; Wang, Z.; Fan, X.; Wang, J. J. Org. Chem. 2015, 80, 10660.

(2) Genna, D. T.; Posner, G. H. Org. Lett. 2011, 13, 5358. 